\title{
Photoproduction of Meson and Baryon Resonances at Energies up to $5.8 \mathrm{GeV}$
}

\author{
AaChen-Berlin-Bonn-Hamburg-Heidelberg-München Collaboration* \\ III. Physikalisches Institut der Technischen Hochschule B, Aachen; Forschungsstelle für Physik hoher \\ Energien der Deutschen Akademie der Wissenschaften zu Berlin-Zeuthen; Physikalisches Institut der Univer- \\ sität Bonn and KFA Jülich, Bonn; Deutsches Elektronen-Synchrotron DESY, Hamburg; Physikalisches \\ Staatinstitut, II. Institut für Experimentalphysik, Hamburg; Institut für Hochenergiephysik der Universität \\ Heidelberg, and Max-Planck-Institut für Physik und Astrophysik, München
}

(Received 26 June 1968)

\begin{abstract}
In a study of photoproduction at photon energies between 0.3 and $5.8 \mathrm{GeV}$ in a hydrogen bubble chamber, approximately 31000 events with three, five, or seven outgoing charged particles were analyzed. This paper gives a survey of the experimental procedure and of resonance production in quasi-two-body reactions. The cross sections for the processes $\gamma p \rightarrow p \pi^{+} \pi^{-}, \gamma p \rightarrow p \pi^{+} \pi^{+} \pi^{-} \pi^{-}, \gamma p \rightarrow p \pi^{+} \pi^{+} \pi^{+} \pi^{-} \pi^{-} \pi^{-}, \gamma p \rightarrow p V\left(V=\rho^{0}\right.$, $\omega, \phi), \gamma p \rightarrow p \eta, \gamma p \rightarrow p X^{0}, \gamma p \rightarrow \Delta^{++} \pi^{-}$, and $\gamma p \rightarrow \Delta^{0} \pi^{+}$have been measured as a function of the photon energy. For reactions $\gamma p \rightarrow p \rho^{0}, \gamma p \rightarrow p \omega$, and $\gamma p \rightarrow \Delta^{++} \pi^{-}$, the differential cross sections and density matrix elements are given for various intervals of the photon energy. The photoproduction of vector mesons and $\Delta$ isobars is discussed in terms of several models.
\end{abstract}

\section{INTRODUCTION AND EXPERIMENTAL PROCEDURE}

\section{A. Introduction}

$T^{\mathrm{H}}$ HIS is the first of two final reports of a bubblechamber experiment to study photoproduction of hadrons at energies up to $5.8 \mathrm{GeV}$. The exposure was made with the $85-\mathrm{cm}$ hydrogen bubble chamber at DESY in a photon beam with a continuous energy spectrum which resembled a thin-target bremsstrahlung spectrum. In this way we were able to study the photoproduction and the decay of mesonic resonances $\left(\rho, \omega, \phi, \eta, X^{0}\right)$ and of baryonic resonances $(\Delta(1236)$, $\Sigma(1385))^{1}$ in various reactions as well as the energy dependence of the cross sections. The smallest cross section which can be studied reasonably with our statistics is about $0.5 \mu \mathrm{b}$.

Preliminary results, based on about one-half of the final statistics, have already been reported. ${ }^{2-8}$ Some

* Group members (by Institution) are: R. Erbe, H. G. Hilpert, E. Schüttler, and W. Struczinski, III. Physikalisches Institut der Technischen Hochschule B, Aachen; K. Lanius, A. Meyer, A. Pose, and H.-J. Schreiber, Forschungsstelle für Physik hoher Energien der Deutschen Akademie der Wissenschaften zu BerlinZeuthen; K. Böckmann, J. Moebes, H. Mück, B. Nellen (now at CERN, Geneva), and W. Tejessy, Physikalisches Institut der Universität Bonn and KFA Jülich, Bonn; G. Horlitz, E. Lohrmann, H. Meyer, W. P. Swanson (Fellow of the Stiftung Volkswagenwerk, now at CERN, Geneva), M. W. Teucher, G. Wolf (now at SLAC, Stanford, Calif.), and S. Wolff, Deutsches Elektronen-Synchrotron DESY, Hamburg; D. Lüke, P. Söding (now at Lawrence Radiation Laboratory, Berkeley, Calif.), H. Spitzer, and F. Storim, Physikalisches Staatinstitut, II. Institut für Experimentalphysik, Hamburg; H. Beisel, H. Filthuth, and P. Steffen, Institut für Hochenergiephysik der Universität Heidelberg; P. Freund, K. Gottstein, N. Schmitz (now at CERN, Geneva), P. Seyboth, and J. Seyerlein, Max-Planck-Institut für Physik und Astrophysik, München.

1 In general, we use the notation of Ref. 41.

2 Aachen-Berlin-Bonn-Hamburg-Heidelberg-München Collaboration, Proceedings of the International Symposium on Electron and Photon Interactions at High Energies, 1965, edited by G. Höhler et al. (Deutsche Physikalische Gesellschaft, Hanau, Germany, 1966), Vol. II, p. 36 (first results).

${ }^{3}$ Aachen-Berlin-Bonn-Hamburg-Heidelberg-München Collaboration, Nuovo Cimento 41A, 270 (1966) (first results). results from the full statistics have been given in Refs. 9 and 10 and have been included in this paper for completeness.

The feasibility of studying high-energy photoproduction in hydrogen by the track-chamber technique was first demonstrated by Sellen et al., ${ }^{11,12}$ using a hydrogendiffusion cloud chamber. In that work the authors studied photoproduction up to about $1 \mathrm{GeV}$ and obtained first results on the production of the $\Delta(1236)$. Later, the production of the $\Delta(1236)$ and of the $\rho^{0}$ meson was studied in a heavy-liquid bubble chamber ${ }^{13}$ and also in a series of counter measurements. ${ }^{14,15}$ With the advent of electron accelerators of higher energies, a much larger number of reactions became

\footnotetext{
4 Aachen-Berlin-Bonn-Hamburg-Heidelberg-München Collaboration, Phys. Letters 23, 707 (1966) $\left[\Delta^{++}(1236)\right.$ production].

5 Aachen-Berlin-Bonn-Hamburg-Heidelberg-München Collaboration, Nuovo Cimento $46 \mathrm{~A}, 795(1966)\left(\omega, \phi, \eta, X^{0}\right.$ production).

6 Aachen-Berlin-Bonn-Hamburg-Heidelberg-München Collabo-
} ration, Nuovo Cimento 48A, 262 (1967); 49A, 337 (1967) ( $\rho$ production).

7 Aachen-Berlin-Bonn-Hamburg-Heidelberg-München Collaboration, Nuovo Cimento 49A, 504 (1967) ; 51A, 246 (1967) (strangeparticle production).

${ }^{8}$ Aachen-Berlin-Bonn-Hamburg-Heidelberg-München Collaboration, Nucl. Phys. B1, 668 (1967); B3, 364 (1967) (particle spectra).

${ }^{9} \mathrm{E}$. Lohrmann, in Proceedings of the 1967 International Symposium on Electron and Photon Interactions at High Energies (Stanford Linear Accelerator Center, Stanford, Calif., 1968), p. 199; DESY Report No. 67/40, 1967 (unpublished).

${ }_{10}$ Aachen-Berlin-Bonn-Hamburg-Heidelberg-München Collaboration, Phys. Letters 27B, 54 (1968) (vector-meson production).

${ }^{11}$ J. M. Sellen, G. Cocconi, V. T. Cocconi, and E. L. Hart, Phys. Rev. 113, 1323 (1959).

${ }_{12}$ B. M. Chasan, G. Cocconi, V. T. Cocconi, R. M. Schectman, and D. H. White, Phys. Rev. 119, 811 (1960).

${ }^{13}$ L. J. Fretwell, Jr., and J. H. Mullins, Phys. Rev. 155, 1497 (1967).

${ }_{14}$ D. McLeod, S. Richert, and A. Silverman, Phys. Rev. Letters 7, 383 (1961); L. J. Lanzerotti, R. B. Blumenthal, D. C. Ehn, W. L. Faissler, P. M. Joseph, F. M. Pipkin, J. R. Randolph, J. J. Russell, D. G. Stairs, and J. Tenenbaum, Phys. Rev. 166, 1365 (1968).

${ }^{15}$ J. V. Allaby, H. L. Lynch, and D. M. Ritson, Phys. Rev. 142, 887 (1966); M. G. Hauser, ibid. 160, 1215 (1967). 


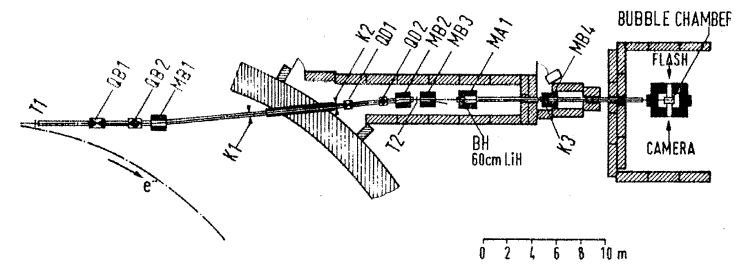

Fig. 1. Experimental layout of the beam and the bubble chamber. T1 and T2 are targets. QB1, QB2, QD1, and QD2 are quadrupole magnets, MB1-MB4 and MA1 are bending magnets. $\mathrm{K} 1-\mathrm{K} 3$ are collimators. $\mathrm{BH}$ is the beam hardener.

accessible, most notably the production of mesonic resonances. An experiment, very similar to the one described here, was carried out at CEA by the Cambridge Bubble Chamber Group. ${ }^{16-23}$

A detailed comparison of their results with ours was given in Ref. 9. Summarizing this comparison, one can say that all experimental distributions of the two collaborations which could be compared were in reasonable agreement. Among other things, agreement was found for total and differential cross sections and the decay angular distributions of all resonances which were observed in both experiments. There are a few differences in the interpretation of the results by the two collaborations, which are discussed in Ref. 9 and, partly, in the respective sections of this paper.

In the following sections we describe the experimental procedure. Total cross sections are given in Sec. II. The results on the photoproduction of the resonances $\rho^{0}, \omega, \phi, \eta, X^{0}, \Delta^{++}(1236)$, and $\Delta^{0}(1236)$ in quasi-twobody reactions are reported and discussed in Secs. III-VIII. A later paper will cover multipion and strangeparticle production.

The reactions $\gamma p \rightarrow p \pi^{0} m \pi^{0}$ and $\gamma p \rightarrow n \pi^{+} m \pi^{0}$ $(m \geq 0)$ which produce one visible prong in the bubble chamber have been analyzed in the first part of the experiment only. Results from the one-prong events have been reported in Ref. 3 and will not be further discussed.

\section{B. Photon Beam}

The layout of the experiment is shown in Fig. 1. The $\boldsymbol{\gamma}$ beam from an internal target of the synchrotron hits

${ }^{16}$ Cambridge Bubble Chamber Group, Phys. Rev. Letters 13, 636 (1964); 13, 640 (1964).

${ }^{17}$ Cambridge Bubble Chamber Group, in Proceedings of the International Symposium on Electron and Photon Interactions at High Energies, 1965, edited by G. Höhler et al. (Deutsche Physikalische Gesellschaft, Hanau, Germany, 1966), Vol. II, p. 1.

${ }_{18}$ Cambridge Bubble Chamber Group, Phys. Rev. 146, 994 (1966) ( $\rho$ production).

${ }_{19}$ Cambridge Bubble Chamber Group, Phys. Rev. 155, 1468 (1967) ( $\omega$ production).

${ }^{20}$ Cambridge Bubble Chamber Group, Phys. Rev. 155, 1477 (1967) (cross sections).

21 Cambridge Bubble Chamber Group, Phys. Rev. 156, 1426 (1967) (strange-particle production).

${ }_{22}$ Cambridge Bubble Chamber Group, Phys. Rev. 163, 1510 (1967) $\left[\Delta^{++}(1236)\right.$ production].

${ }^{23}$ Cambridge Bubble Chamber Group, Phys. Rev. 169, 1081 (1968) (multipion production). an external target $\mathrm{T} 1$, where an electron beam is started by conversion. The first bending magnet MB1 and a collimator define the momentum of this electron beam to $\Delta p / p= \pm 1 \%$. The electron beam strikes a second target $\mathrm{T} 2$ between the bending magnets MB2 and MB3. Bremsstrahlung from this target passes through a $\mathrm{LiH}$ beam hardener, consisting of $60-\mathrm{cm}$ $\mathrm{LiH}$ in a magnetic field of $8.6 \mathrm{kG}$. The beam hardener was used in order to reduce the fraction of low-energy photons $\left(E_{\gamma} \lesssim 20 \mathrm{MeV}\right)$ in the beam. (We also made trial runs without the beam hardener and obtained acceptable pictures.) Magnets MB3, MA1, and MB4 clean the beam of charged particles.

The photon-beam intensity was adjusted to about 65 effective quanta/picture by changing the width of the collimators and the thickness of the conversion targets. This flux produced an average of ten electronpositron pairs per picture in the scanning volume of the bubble chamber.

Approximately 1.7 million pictures were taken. In the exposure of the first 350000 pictures, the maximum photon energy $E_{\gamma}{ }^{\max }$ was $5.45 \mathrm{GeV}$, and the mean thickness of the second conversion target T2 was 0.065 radiation length. The rest of the film was taken with $E_{\gamma}{ }^{\max }=5.8 \mathrm{GeV}$ and a mean target thickness of 0.1 radiation length.

The properties of the resulting photon beam, as determined by the measurement of about 30000 electron-positron pairs in the bubble chamber, were the following:

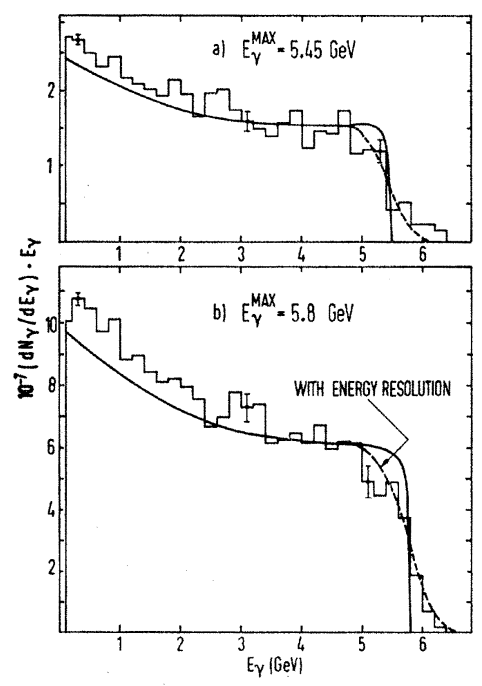

Fig. 2. Photon spectra for the two parts of the experiment: (a) $E_{\gamma}{ }_{\max }^{2}=5.45 \mathrm{GeV}$, conversion target $\mathrm{T} 2$ of $X=0.065$ radiation length; (b) $E_{\gamma}{ }^{\max }=5.8 \mathrm{GeV}, X=0.1$. The error bars indicate the statistical errors. The experimental points are normalized to a total flux of $9.1 \times 10^{7}$ equivalent quanta with $E_{\gamma}>0.1 \mathrm{GeV}$, corresponding to the number of events in Table I. The full curves are thin-target bremsstrahlung spectra with corrections for finite target thickness and collimation of the photon beam (Refs. 24 and 25). The dashed curves include the energy resolution. Both curves are normalized to the experimental spectrum above $3 \mathrm{GeV}$. 
(a) Beam shape: The photon beam had a cross section of $\Delta y \times \Delta z=7.5 \times 3.5 \mathrm{~cm}^{2}$ at the bubble chamber, where $\Delta y$ and $\Delta z$ are the full widths at half-height in directions parallel and perpendicular to the film plane, respectively.

(b) Beam direction: The photon beam was parallel within our measuring accuracy. The incident beam direction was determined as the average direction of pairs with energy $E_{\gamma}>0.5 \mathrm{GeV}$. The angular uncertainty (rms) of a single pair was $2 \mathrm{mrad}$ in the film plane and $3.5 \mathrm{mrad}$ perpendicular to the film plane.

(c) Energy spectrum: The photon energy spectrum was determined from the measured pair spectrum using the known cross section for pair production in hydrogen (see Sec. I F). The spectra for the two different beam energies are shown in Fig. 2. The full curve in Fig. 2 is a thin-target bremsstrahlung spectrum taken from Ref. 24 with a correction for finite target thickness. ${ }^{25}$ The dashed curve takes into account our limited measuring accuracy, which is $\pm 6 \%$ at $5.5 \mathrm{GeV}$. The

TABLE I. (A) Number of events found in two scans. (B) Number of events corrected for scanning losses. The numbers of 3- and 5 -prong events given in the table correspond to a flux of $9.1 \times 10^{7}$ equivalent quanta with $E_{\gamma}>0.1 \mathrm{GeV}$. The 7-prong events correspond to $8.6 \times 10^{7}$ equivalent quanta. The scanning volume used had a length of $45 \mathrm{~cm}$.

\begin{tabular}{|c|c|c|c|}
\hline \multirow{2}{*}{$\begin{array}{c}\text { Hypothesis considered } \\
\text { by GRIND }\end{array}$} & \multirow{2}{*}{$\begin{array}{l}\text { Number of } \\
\text { constraints }\end{array}$} & \multicolumn{2}{|c|}{ Number of events } \\
\hline & & (A) Found & (B) Corrected \\
\hline \multirow{4}{*}{$\begin{array}{ll}\text { 1. } & \gamma p \rightarrow p \pi^{+} \pi^{-} \\
\text {2. } & \gamma p \rightarrow p \pi^{+} \pi^{-} \pi^{0} \text { a } \\
\text { 3. } & \gamma p \rightarrow n \pi^{+} \pi^{+} \pi^{-\mathrm{a}} \\
\text { 4. } & \text { Ambiguous between } \\
& \text { hypotheses } 2 \text { and } 3 \mathrm{a}\end{array}$} & 3 & 18780 & 19020 \\
\hline & 0 & 6770 & 6800 \\
\hline & 0 & 3020 & 3050 \\
\hline & 0 & 1310 & 1330 \\
\hline \multirow{3}{*}{$\begin{array}{ll}\text { 5. } & \gamma p \rightarrow p \pi^{+} \pi^{+} \pi^{-} \pi^{-} \\
\text {6. } & \gamma p \rightarrow p \pi^{+} \pi^{+} \pi^{-} \pi^{-} \pi^{0 \mathrm{a}} \\
\text { 7. } & \gamma p \rightarrow n \pi^{+} \pi^{+} \pi^{+} \pi^{-} \pi^{-\mathrm{a}}\end{array}$} & 3 & 590 & 590 \\
\hline & 0 & 600 & 600 \\
\hline & 0 & 250 & 250 \\
\hline $\begin{array}{l}\text { 8. Ambiguous between } \\
\text { hypotheses } 6 \text { and } 7 \mathrm{a}\end{array}$ & 0 & 200 & 200 \\
\hline 9. $\gamma p \rightarrow p 3 \pi^{+} 3 \pi^{-}$ & 3 & 18 & 18 \\
\hline \multirow{2}{*}{$\begin{array}{l}\text { 10. } \gamma p \rightarrow p 3 \pi^{+} 3 \pi^{-} \pi^{0 a} \\
\text { 11. } \gamma p \rightarrow n 4 \pi^{+} 3 \pi^{-a}\end{array}$} & 0 & 16 & 16 \\
\hline & 0 & 7 & 7 \\
\hline 12. $\gamma p \rightarrow p K^{+} K^{-}$ & 3 & 104 & 105 \\
\hline $\begin{array}{l}\text { 13. Ambiguous between } \\
\text { hypotheses } 1 \text { and } 12\end{array}$ & 3 & 8 & 8 \\
\hline \multirow{2}{*}{$\begin{array}{l}\text { 14. } \gamma p \rightarrow p K^{0} \bar{K}^{0 \mathrm{a}} \\
\text { 15. Ambiguous between } \\
\text { hypothesis } 14 \text { and } \\
\text { other hypotheses }\end{array}$} & 0 & 23 & \\
\hline & 0 & 11 & \\
\hline $\begin{array}{l}\text { 16. Remaining strange- } \\
\text { particle hypotheses }\end{array}$ & & 1152 & \\
\hline \multicolumn{4}{|l|}{ Unassigned events: } \\
\hline 3-prong events & \multicolumn{2}{|c|}{$\begin{array}{l}\text { No fit with proton } \\
\text { No fit without proton } \\
\text { Unmeasurable }\end{array}$} & $\begin{array}{r}427 \\
16 \\
510\end{array}$ \\
\hline 5-prong events & it & & $\begin{array}{l}18 \\
89\end{array}$ \\
\hline 7-prong events & it & & $\begin{array}{r}1 \\
10\end{array}$ \\
\hline
\end{tabular}

a The events from these reactions are contaminated by events with two or more unobserved secondary neutrals.

A detailed list of strange-particle events will be given in a second paper.

${ }^{24}$ H. D. Schulz, DESY Report No. 66/16, 1966 (unpublished).

${ }^{26}$ G. Lutz and H. D. Schulz, DESY Report No. 67/29, 1967 (unpublished).

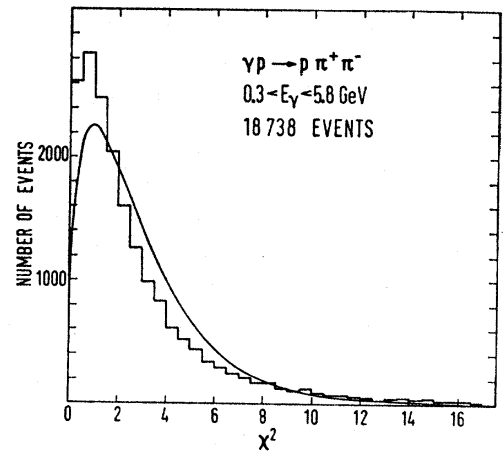

FIG. 3. Distribution of $\chi^{2}$ obtained from kinematical fits of events $\gamma p \rightarrow p \pi^{+} \pi^{-}$. The curve is the theoretical $\chi^{2}$ distribution for three degrees of freedom. The shift of the experimental distribution to lower values of $\chi^{2}$ is due to a slight overestimation of the measuring errors by the reconstruction programs.

theoretical curves are normalized to the experimental spectrum above $3 \mathrm{GeV}$. The deviations from the calculated spectrum at lower energies are mainly due to the beam hardener.

\section{Bubble Chamber}

The $85-\mathrm{cm}$ bubble chamber at DESY is an improved version of the Saclay $81-\mathrm{cm}$ bubble chamber. The dimensions are $85 \mathrm{~cm} \times 40 \mathrm{~cm} \times 40 \mathrm{~cm}$. The illuminated volume covers $70 \mathrm{~cm} \times 34 \mathrm{~cm} \times 40 \mathrm{~cm}$. The beam enters the chamber through two windows, one of $0.5-\mathrm{mm}$ stainless steel and one of 3-mm aluminum. Particles produced in the first window with momenta below $2 \mathrm{GeV} / c$ are swept out by a clearing magnet. The chamber was located in a magnetic field of about $22 \mathrm{kG}$.

\section{Scanning and Measuring}

All the films were scanned twice. In this way we obtained a scanning efficiency of $\geq 99 \%$ for three and more prong events. Some special topologies, e.g., 3 prongs with invisible proton and 1 prong with $V^{0}$, are more difficult to detect and the corresponding cross sections have been corrected separately.

The scanning volume had a mean length of $45 \mathrm{~cm}$. The minimum track length available for measurement was about $16 \mathrm{~cm}$ in the forward direction and $10 \mathrm{~cm}$ in the backward direction. The events were measured on digitized measuring projectors. The geometrical reconstruction was done in the six laboratories by three different geometry programs. The results from all groups were compared and were found to agree with one another.

\section{E. Kinematic Analysis and Event Identification}

\section{Evaluation of Hypotheses}

The kinematical reconstruction was done with the CERN program "GRIND." The interaction hypotheses considered by GRIND are given in Table I. 
In our experiment, the direction but not the energy of the incident photon is known. For hypotheses for which all outgoing particles are observed the photon energy can be calculated from energy and momentum balance (four equations) and one is left with a 3-constraint (3C) fit (hypotheses 1, 5, 9, and 12 of Table I). Figure 3 shows the $\chi^{2}$ distribution for events of the type $\gamma p \rightarrow p \pi^{+} \pi^{-}$. For comparison, we give the theoretical $\chi^{2}$ distribution for three degrees of freedom (full curve).

Considering hypotheses with unobserved neutral particles, one can calculate the energy of the incident photon and the momentum vector of the neutral particle or system of neutral particles, after having fixed its mass. Since no additional equation is then left which enables a kinematical fit $(0 \mathrm{C})$, one cannot distinguish events with one neutral particle (singleneutral events) from events with two and more neutral particles (multineutral events). Therefore reactions of the type

$$
\gamma p \rightarrow p \pi^{+} \pi^{-} \pi^{0} \pi^{0},
$$

for example, were analyzed as if only a single $\pi^{0}$ were produced. In most of these cases the photon energy evaluated by GRIND comes out too low. The photon energy of reactions with a single neutral particle is calculated correctly. Thus we are able to study resonance production from single-neutral events, e.g.,

$$
\gamma p \rightarrow p \omega \rightarrow p \pi^{+} \pi^{-} \pi^{0} .
$$

In general, multineutral events contribute to the background below resonances, which are produced in single-neutral reactions. ${ }^{19}$

\section{Event Identification}

The selection between the hypotheses which were kinematically acceptable according to GRIND was made in the following way:

(1) The agreement of the calculated and the observed ionization of all tracks was checked. (We could separate pions from protons up to about 1.4 $\mathrm{GeV} / c$ on the scanning table.) In case of disagreement the respective hypothesis was disregarded.

The remaining hypotheses were treated as follows:

(2) All 3C hypotheses with a probability greater than 0.001 were accepted. If there was a $0 \mathrm{C}$ hypothesis in addition, this hypothesis was neglected.

(3) If an event did not have an accepted 3C fit, all remaining $0 \mathrm{C}$ hypotheses were accepted. Events with two or more 0C hypotheses were classified as ambiguous.

A small number of events gave no acceptable hypothesis (no-fit events). Few events were unmeasurable for geometrical reasons. Column A of Table I shows the number of events obtained from the event identification. Column $\mathrm{B}$ gives the numbers of events after corrections for scanning losses.

We have looked for possible biases in the kinematic reconstruction and event identification procedure by inspecting the experimental $\chi^{2}$ distributions and by making detailed Monte Carlo calculations with the program FAKE. ${ }^{26}$ The result was:

(a) 3C Reactions: The percentage of events which are wrongly interpreted is less than $1 \%$.

(b) 0C Reactions: The fraction of wrongly interpreted events is about $1 \%$.

A special inaccuracy occurs in events of the type

$$
\gamma p \rightarrow p \pi^{+} \pi^{-} \pi^{0}
$$

with a forward-going $\pi^{0}$. For these events small errors in the measured transverse momenta lead to large errors in the calculated longitudinal momenta of the $\pi^{0}$ and of the photon. If the $\pi^{0}$ originates from a resonance, the resonance peak will be smeared out due to the shift in the $\pi^{0}$ momentum. We estimate from FAKE calculations that for $10-15 \%$ of all events $\gamma p \rightarrow p \pi^{+} \pi^{-} \pi^{0}$ the error of the $\pi^{0}$ and photon energy is noticeable. The $\omega$ and $\eta$ cross sections are corrected for this effect.

\section{F. Calculation of Cross Sections}

The cross sections for hadron production were obtained by relating the number of hadronic events to the number of $e^{+} e^{-}$pairs observed in the same scanning volume, using the cross section for $e^{+} e^{-}$pair production from theory.

\section{Cross Section for Electron-Positron Pair Production}

The cross section for coherent and incoherent pair production on hydrogen was calculated in the Born approximation according to Wheeler and Lamb (WL). ${ }^{27}$ We used the formulas of WL for $\epsilon \leq 1.5$ and $\gamma \leq 1.5$, where $\gamma$ and $\epsilon$ are the screening parameters defined in Ref. 27. For $\gamma>1.5$ and $\epsilon>1.5$, Eq. (5) of Ref. 28 was taken. The results are given in Table II.

Four types of corrections must be considered:

(1) Molecular effects due to differences between the atomic wave function used by WL and the wave function of the real $\mathrm{H}_{2}$ molecule have been estimated for bremsstrahlung by Bernstein and Panofsky $(+2.7 \%){ }^{29}$ Bernstein and Panofsky applied wave functions from the Heitler-London approximation. Calculations for pair production with various other molecular wave

${ }^{26}$ G. R. Lynch, University of California Lawrence Radiation Laboratory Report No. UCRL-10335 (unpublished); E. Raubold, revised version of FAKE (private communication).

${ }^{27} \mathrm{~J}$. A. Wheeler and W. E. Lamb, Jr., Phys. Rev. 55, 858 (1939); ibid. 101, 1836 (1956).

${ }_{28}$ B. Rossi, High Energy Particles (Prentice-Hall, Inc., Englewood Cliffs, N. J., 1965), p. 80.

${ }^{29}$ D. Bernstein and W. K. H. Panofsky, Phys. Rev. 102, 522 (1956). 
functions are in $\operatorname{progress}^{30}$ and tend to give much lower values for the correction.

(2) The contributions of exchange effects and $\gamma e$ interaction have been estimated by Joseph and Rohrlich. ${ }^{31}$ They get an energy-independent correction of $-16 \%$ to the incoherent cross section of WL. However, Suh and Bethe ${ }^{32}$ have shown that the effects should vanish as $\ln \left(E_{\gamma}\right) / E_{\gamma}$. A recent calculation by Mork ${ }^{33}$ confirms the results of Suh and Bethe. Mork has found explicitly that the contributions of both effects cancel above $10 \mathrm{MeV}$.

Thus molecular effects, exchange effects, and $\gamma e$ interaction can be neglected.

(3) The retardation effect in incoherent pair production is included in Mork's calculations. It leads to a correction of the WL total cross sections of $-1.5 \%$ at $0.5 \mathrm{GeV}$ and decreases with increasing energy. ${ }^{30}$

(4) Mork and Olsen have calculated the radiative corrections to pair production, and get a $+0.93 \%$ contribution to the high-energy cross section. ${ }^{34}$

In the present experiment we do not account for the retardation effect or radiative corrections. Thereby a small uncertainty of about $1 \%$ is introduced. Apart from the above corrections the use of the WL formulas should be correct to within $1 \%$, above $0.3 \mathrm{GeV}$. Both errors are small compared to the statistical errors.

\section{Flux Determination and Calculation of Cross Sections}

The photon energy spectrum was calculated from the measured pair spectrum and the pair-production cross section. The total photon flux has been determined from counting $e^{+} e^{-}$pairs with total energies greater than $50 \mathrm{MeV}$ on every 100th good picture. The flux

TABLE II. Cross sections for coherent $\left(\sigma_{p}\right)$ and incoherent $\left(\sigma_{e}\right)$ pair production on hydrogen according to Wheeler and Lamb and to Rossi (see text).

\begin{tabular}{cccc}
\hline $\begin{array}{c}E_{\gamma} \\
(\mathrm{GeV})\end{array}$ & $\begin{array}{c}\sigma_{p} \\
(\mathrm{mb})\end{array}$ & $\begin{array}{c}\sigma_{e} \\
(\mathrm{mb})\end{array}$ & $\begin{array}{c}\sigma_{n}+\sigma_{e} \\
(\mathrm{mb})\end{array}$ \\
\hline 0.1 & 6.09 & 6.09 & 12.19 \\
0.15 & 6.76 & 6.81 & 13.57 \\
0.2 & 7.15 & 7.30 & 14.45 \\
0.25 & 7.45 & 7.66 & 15.11 \\
0.3 & 7.66 & 7.95 & 15.61 \\
0.4 & 7.97 & 8.39 & 16.36 \\
0.5 & 8.19 & 8.70 & 16.88 \\
0.7 & 8.45 & 9.12 & 17.57 \\
1.0 & 8.69 & 9.50 & 18.20 \\
1.5 & 8.90 & 9.86 & 18.77 \\
2.0 & 9.00 & 10.07 & 19.08 \\
3.0 & 9.11 & 10.30 & 19.42 \\
4.0 & 9.17 & 10.43 & 19.60 \\
5.0 & 9.21 & 10.51 & 19.72 \\
6.0 & 9.23 & 10.57 & 19.80 \\
\hline \hline
\end{tabular}

${ }^{30}$ T. M. Knasel, thesis, Harvard University, 1967 (unpublished); and private communication.

${ }_{31}$ J. Joseph and F. Rohrlich, Rev. Mod. Phys. 30, 354 (1958).

${ }^{32}$ K. S. Suh and H. A. Bethe Phys. Rev. 115, 672 (1959).

${ }^{33}$ K. J. Mork, Phys. Rev. 160, 1065 (1967).

${ }^{34}$ K. J. Mork and H. Olsen, Phys. Rev. 140, B1661 (1965).

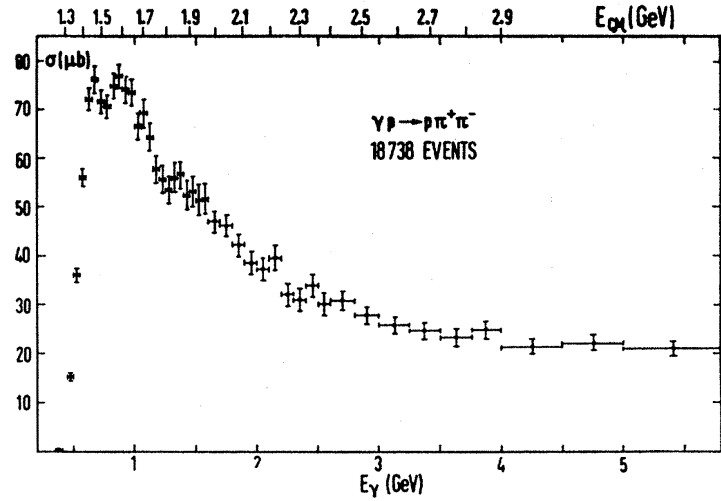

FIG. 4. Total cross section for the reaction $\gamma p \rightarrow p \pi^{+} \pi^{-}$as a function of the photon energy $E_{\gamma}$ in the lab system. $E_{\mathrm{c} . \mathrm{m} \text {. is the }}$ total energy in the center-of-mass system.

was corrected for scanning losses $(0.5 \%)$ and for pairs produced by nonbeam photons outside the beam region (1\%). The photon-energy spectrum is shown in Figs. 2(a) and 2(b). The total flux was $9.1 \times 10^{7}$ equivalent quanta. It was calculated by integrating the energy spectra of Fig. 2 above $0.1 \mathrm{GeV}$ and then dividing by $E_{\gamma}{ }^{\max }$. The uncertainties of the photon spectrum due to limited statistics are $2 \%$ below $3.5 \mathrm{GeV}$, about $5 \%$ for $3.5 \mathrm{GeV}<E_{\gamma}<5.0 \mathrm{GeV}$, and about $10 \%$ for $E_{\gamma}>5$ $\mathrm{GeV}$.

For the calculation of cross sections, the unmeasurable events of Table I were distributed among the different channels in the ratio of the measurable events. The three-prong no-fit events with a proton were divided among the reactions (1a), (2a), and ( $\gamma p \rightarrow p e^{+} e^{-}$ or $\gamma p \rightarrow p \mu^{+} \mu^{-}$) in the ratio 0.25:0.35:0.40 according to FAKE results and inspection of the effective-mass distributions.

A more detailed description of the experimental procedure is given in Ref. 35 .

\section{TOTAL CROSS SECTIONS}

Figure 4 shows the total cross section for the reaction

$$
\gamma p \rightarrow p \pi^{+} \pi^{-}
$$

as a function of the photon energy $E_{\gamma}$. Upper limits for the cross sections of the reactions

$$
\begin{aligned}
& \gamma p \rightarrow p \pi^{+} \pi^{-} \pi^{0}, \\
& \gamma p \rightarrow n \pi^{+} \pi^{+} \pi^{-}
\end{aligned}
$$

are shown in Fig. 5 as functions of $E_{\gamma}$. The points in this figure are upper limits for the following reason:

The values were obtained from all events which were consistent with hypothesis (2) or (3), respectively (see Sec. I E), including all ambiguous events (Table I). The points therefore certainly contain all events which truly belong to reaction (2a) or (3) and for which the

${ }^{35}$ H. Spitzer, DESY Internal Report No. F1/4, 1967; and Stanford Linear Accelerator Center Report No. SLAC Trans-79, 1968 (unpublished). 

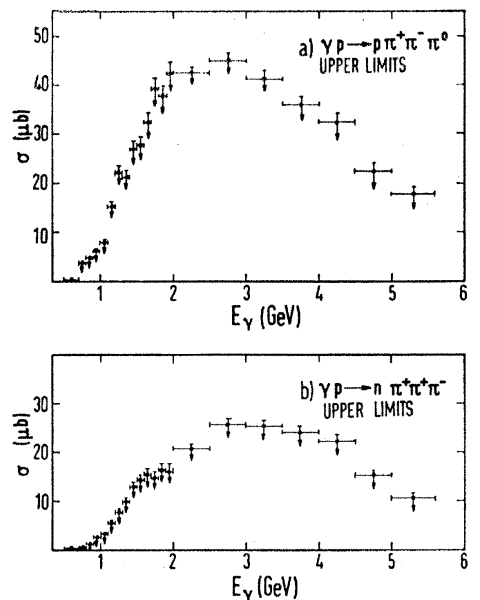

FIG. 5. Upper limits for the cross sections of the reactions (a) $\gamma p \rightarrow p \pi^{+} \pi^{-} \pi^{0}$ and (b) $\gamma p \rightarrow n \pi^{+} \pi^{+} \pi^{-}$as functions of the photon energy $E_{\gamma}$. The points contain all events from categories (2) and (4) of Table I (for $\gamma p \rightarrow p \pi^{+} \pi^{-} \pi^{0}$ ) and all events of categories (3) and (4) (for $\gamma p \rightarrow n \pi^{+} \pi^{+} \pi^{-}$).

primary momentum could be determined correctly. In addition, the points include events with more than one neutral particle which cannot be distinguished from events with one neutral secondary.

The Cambridge Bubble Chamber Group ${ }^{20}$ has tried to separate the multineutral from the single-neutral events by analyzing the laboratory spectra of threeand five-prong events. They find that their sample of hypothesis (2) contains about $50 \%$ multineutral events [the same fraction was used for hypothesis (3)]. Therefore the cross sections for reactions (2a) and (3) given in Ref. 20 are much lower than our upper limits. We have not attempted to separate the multineutral events, since accurate cross-section measurements for the reactions $(2 a),(3),(6)$, and (7) will soon be possible with monoenergetic photon beams.

Figures 6(a) and 6(d) show the cross sections for the reactions

$$
\begin{aligned}
& \gamma p \rightarrow p \pi^{+} \pi^{+} \pi^{-} \pi^{-}, \\
& \gamma p \rightarrow p \pi^{+} \pi^{+} \pi^{+} \pi^{-} \pi^{-} \pi^{-} .
\end{aligned}
$$

The points labeled " $\pi N$ data" in Figs. (6a) and (6d) represent theoretical predictions by Satz. ${ }^{36}$ Satz combined the vector-dominance model, the quark model, and the statistical model to predict cross sections for reactions (4) and (5) from the experimental cross sections for the corresponding reactions $\pi^{ \pm} p \rightarrow N \pi \pi \pi \pi$ and $\pi^{+} p \rightarrow p \pi \pi \pi \pi \pi \pi$, respectively. There is good agreement between the predictions of Satz and our experimental values.

In Figs. 6(b) and 6(c) upper limits are given for the cross sections of the reactions

$$
\begin{aligned}
& \gamma p \rightarrow p \pi^{+} \pi^{+} \pi^{-} \pi^{-} \pi^{0}, \\
& \gamma p \rightarrow n \pi^{+} \pi^{+} \pi^{+} \pi^{-} \pi^{-} .
\end{aligned}
$$

${ }^{36}$ H. Satz, Phys. Letters 25B, 27 (1967).
The errors given in Figs. 4-6 are statistical. The systematic errors in the cross sections for reactions (1a), (4), and (5) due to absolute normalization are $\lesssim 5 \%$ for $E_{\gamma}<5 \mathrm{GeV}$ and about $10 \%$ for $E_{\gamma}>5 \mathrm{GeV}$.

\section{THE REACTION $\gamma p \rightarrow p 0^{0}$}

\section{A. Mass Distributions and Fitting Procedure}

The reaction

$$
\gamma p \rightarrow p \pi^{+} \pi^{-}
$$

is dominated by $\Delta^{++}(1236)$ production at lower energies and by $\rho$ production at higher energies. Figures 7 (a) and 7(b) show distributions of the $\pi^{+} \pi^{-}$effective mass $M\left(\pi^{+} \pi^{-}\right)$for twelve intervals of $E_{\gamma}$. While no resonant structure is present at lower energies, $\rho^{0}$ production is clearly observed above $E_{\gamma}=1.1 \mathrm{GeV}$.

For obtaining the total and differential production cross sections for the reaction

$$
\begin{aligned}
& \gamma p \rightarrow p p^{0} \\
& \searrow \\
& \pi^{+} \pi^{-},
\end{aligned}
$$

the following fitting procedure was used:

The density distribution $d N\left(M^{2}\left(p \pi^{+}\right), M^{2}\left(\pi^{+} \pi^{-}\right)\right)$of events in the Dalitz plot $M^{2}\left(p \pi^{+}\right)$versus $M^{2}\left(\pi^{+} \pi^{-}\right)$

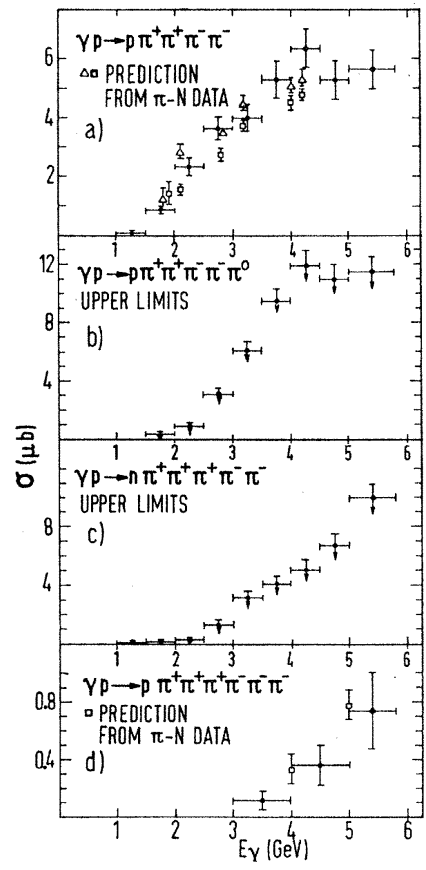

Fig. 6. Cross sections for five- and seven-prong events as a function of the photon energy $E_{\gamma \cdot}$. (a) Cross section for $\gamma p \rightarrow$ $p \pi^{+} \pi^{+} \pi^{-} \pi^{-}$. (b) and (c) Upper limits for the cross sections for reactions $\gamma p \rightarrow p \pi^{+} \pi^{+} \pi^{-} \pi^{-} \pi^{0}$ and $\gamma p \rightarrow n \pi^{+} \pi^{+} \pi^{+} \pi^{-} \pi^{-}$, respectively. The points contain all events from categories (6) and (8) of Table I (for $\gamma p \rightarrow p \pi^{+} \pi^{+} \pi^{-} \pi^{-} \pi^{0}$ ) and all events of categories (7) and (8) (for $\gamma p \rightarrow n \pi^{+} \pi^{+} \pi^{+} \pi^{-} \pi^{-}$). (d) Cross section for $\gamma p \rightarrow p \pi^{+} \pi^{+} \pi^{+} \pi^{-} \pi^{-} \pi^{-}$. The points labeled "prediction from $\pi N$ data" are taken from calculations by Satz (Ref. 36) (see text). 
FIG. 7. Reaction $\gamma p \rightarrow p \pi^{+} \pi^{-}$ (a) Effective-mass distributions $M\left(\pi^{+} \pi^{-}\right)$for seven intervals of the photon energy below 1.1 $\mathrm{GeV}$. The curves are the sum of the contributions from Lorentzinvariant phase space and the reflections from $\gamma p \rightarrow \Delta^{++} \pi^{-}$ and $\gamma p \rightarrow \Delta^{0} \pi^{+}$including interference between $\Delta^{++}$and $\Delta^{0}$ amplitudes. For $1.0<E_{\gamma}<1.1$ $\mathrm{GeV}$ the curve contains also the resonance distribution for $\rho^{0}$. The curves were obtained by the fitting method described in Sec. VIII A. (b) Distributions of $M\left(\pi^{+} \pi^{-}\right)$for five intervals above $1.1 \mathrm{GeV}$. Meaning of the curves: - - - - Superposition of $\rho$-resonance distribution (i), Lorentz-invariant phase space and $\Delta^{++}(1236)$ reflection (see Sec. III A). - - The same but with distribution (ii).

The same but with distribution (iii). ... Sum of phase space and $\Delta^{++}(1236)$ reflection from method (i) $\left(1.1<E_{\gamma}<1.4 \mathrm{GeV}\right)$ and method (ii) $\left(E_{\gamma}>1.4 \mathrm{GeV}\right)$, respectively.
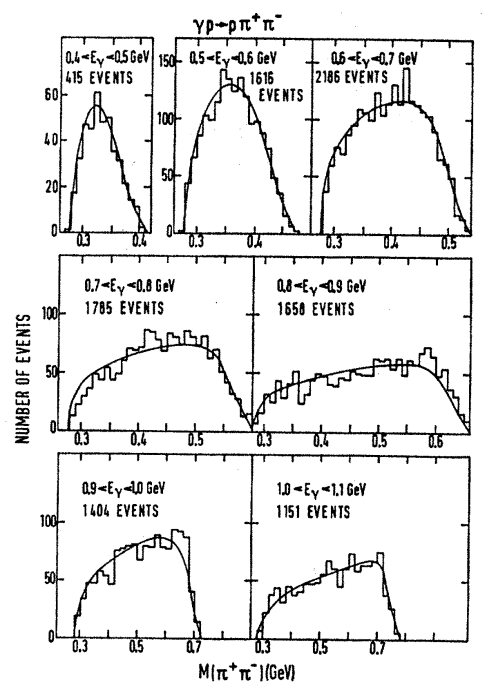

(a)
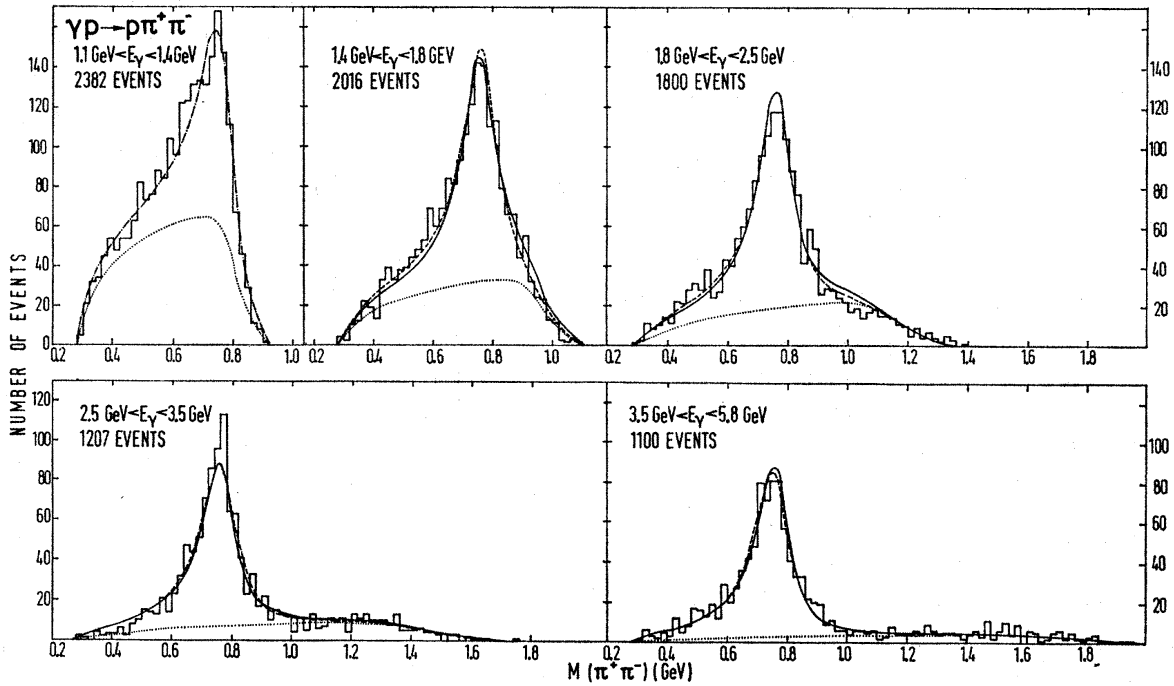

(b) was assumed to be a sum of three noninterfering terms,

$$
\begin{aligned}
& d N\left(M^{2}\left(p \pi^{+}\right), M^{2}\left(\pi^{+} \pi^{-}\right)\right)=\left[a_{\Delta} f_{\Delta}\left(M\left(p \pi^{+}\right)\right)\right. \\
& \left.+a_{\rho} f_{\rho}\left(M\left(\pi^{+} \pi^{-}\right)\right) W\left(\cos \theta_{H}\right)+a_{\mathrm{PS}} f_{\mathrm{PS}}\right] \\
& \times\left(1 / E_{\mathrm{c.m} .}{ }^{2} E_{\gamma}\right) d M^{2}\left(p \pi^{+}\right) d M^{2}\left(\pi^{+} \pi^{-}\right) \\
& M\left(p \pi^{+}\right)=p \pi^{+} \text {effective mass, } \\
& E_{\mathrm{c} . \mathrm{m} .}=\text { total energy in the center-of-mass system. }
\end{aligned}
$$

The parameters $a_{\Delta}, a_{\rho}$, and $a_{\mathrm{PS}}$ are the contributions (number of events) of $\Delta^{++}, \rho^{0}$, and background production to be determined. $f_{\Delta}$ and $f_{\rho}$ are resonance distributions for $\Delta^{++}$and $\rho^{0}$ production. $f_{\mathrm{PS}}$ is the Lorentzinvariant phase-space distribution. $W\left(\cos \theta_{H}\right)$ describes the $\rho$ decay distribution in the helicity system (see below). In this system one has a linear relation between the cosine of the decay angle $\cos \theta_{H}$ and $M^{2}\left(p \pi^{+}\right)$. The distributions $f_{\Delta}, f_{\rho} \cdot W$, and $f_{\mathrm{PS}}$ are each normalized to unity when integrated over the whole Dalitz plot.
The parameters were determined both by least-squares and maximum-likelihood fits. The inclusion of terms describing $f^{0}$ and $\Delta^{0}$ production had a negligible effect on the $\rho$ cross section. Details of the fitting procedure are given in the Appendix and in Ref. 35 .

Three choices for the form $f_{\rho}$ of the $\pi^{+} \pi^{-}$mass distribution resulting from $\rho$ decay were tried in the fits:

(i) A Breit-Wigner distribution with an energydependent width according to Jackson. ${ }^{37}$

(ii) A Breit-Wigner distribution as in (i), multiplied by a term $\left(M_{\rho} / M\left(\pi^{+} \pi^{-}\right)\right)^{4}$. This factor was proposed by Ross and Stodolsky ${ }^{38}$ to account for the diffractive character of the $\rho^{0}$ production in the framework of a vector-dominance model.

${ }^{37}$ J. D. Jackson, Nuovo Cimento 34, 1644 (1964).

${ }^{38}$ M. Ross and L. Stodolsky, Phys. Rev. 149, 1172 (1966). 


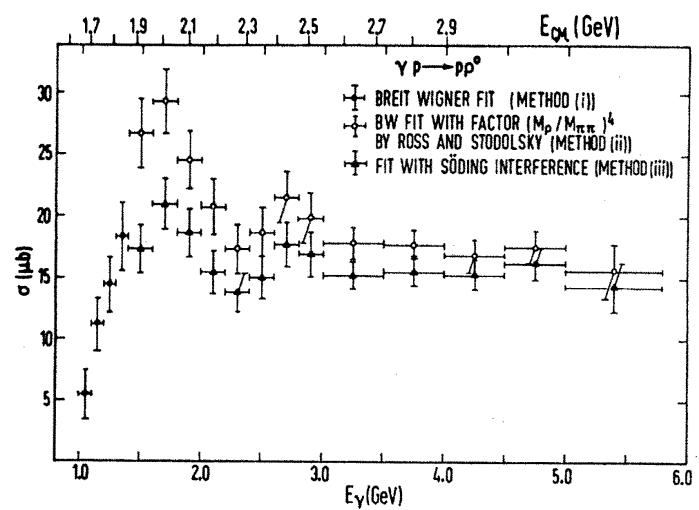

FIg. 8. Total cross section for the reaction $\gamma p \rightarrow p \rho^{0}$ as a function of the photon energy $E_{\gamma}$. The different points were obtained by three fitting methods as indicated in the figure (see Sec. III A).

(iii) A Breit-Wigner distribution as in (i) plus a Drell-type background interfering with the BreitWigner amplitude. ${ }^{39}$ The relative phase of the $\rho$ amplitude and the background amplitude was fixed, ${ }^{40}$ whereas the amount of background was fitted.

Below $E_{\gamma}=1.4 \mathrm{GeV}$, distribution (i) gave acceptable fits to the data. Above $1.4 \mathrm{GeV}$, the fit with (i) was poor and gave too low values for the $\rho$ mass. Method (ii) gave acceptable fits above $1.4 \mathrm{GeV}$ [see broken curves in Fig. 7(b)] and values for the $\rho$ mass, which decreased slightly with increasing photon energy from $(778 \pm 4)$ to $(762 \pm 5) \mathrm{MeV}$. This slight variation is an indication that the Ross-Stodolsky diffraction model does not describe all of our $\rho$ production data. Obviously, fitting data at $E_{\gamma}$ as low as $1.4 \mathrm{GeV}$ and without restricting the production angle to the forward diffraction region might stress the limits of validity of a pure diffraction-type model too much.

Method (iii) gave acceptable fits above $1.4 \mathrm{GeV}$ [see full curves in Fig. 7(b) ] and values for the $\rho$ mass between $(759 \pm 6)$ and $(783 \pm 5) \mathrm{MeV}$ with an average of $770 \mathrm{MeV}^{41}$ and no systematic dependence on the photon energy. The width of the $\rho$ meson was kept fixed at $143 \mathrm{MeV}$ for all three fits. (This value was obtained as an average from several preliminary fits with adjustable mass and width for the $\rho$ meson at $E_{\gamma}$ above $1.8 \mathrm{GeV}$.) Our mass resolution of $\pm 7 \mathrm{MeV}$ at $M\left(\pi^{+} \pi^{-}\right)=770 \mathrm{MeV}$ does not contribute considerably to the fitted width.

\footnotetext{
${ }^{39}$ P. Söding, Phys. Letters 19, 702 (1965).

${ }^{40} \mathrm{It}$ was assumed that at the resonance $\left[M\left(\pi^{+} \pi^{-}\right)=770 \mathrm{MeV}\right]$ the $\rho$ production amplitude is purely imaginary, corresponding to a diffractionlike production process. The interference of the $\rho$ amplitude, having a rapidly varying phase in the $\rho$ resonance region, with a background amplitude of relatively slowly varying phase leads to a distortion and displacement of the $\rho$ resonance peak. It is easy to show that if one in addition assumes vector dominance, the $\rho$ resonance peak will appear displaced downward in mass.

${ }_{41}$ A. H. Rosenfeld, N. Barash-Schmidt, A. Barbaro-Galtieri, L. R. Price, M. Roos, P. Söding, W. J. Willis, and C. G. Wohl, Rev. Mod. Phys. 40, 77 (1968).
}

\section{B. Cross Sections}

In the fits performed to determine cross sections, the $\rho$ mass was kept fixed as follows: methods (i) and (iii), $M_{\rho}=770 \mathrm{MeV}$; method (ii), $M_{\rho}=778 \mathrm{MeV}$ for $1.4<E_{\gamma}$ $<3.5 \mathrm{GeV}, M_{\rho}=770 \mathrm{MeV}$ for $3.5<E_{\gamma}<4.5 \mathrm{GeV}$, $M_{\rho}=762 \mathrm{MeV}$ for $4.5<E_{\gamma}<5.8 \mathrm{GeV}$. Figure 8 shows the $\rho$-production cross section as a function of $E_{\gamma}$. For $E_{\gamma}<1.4 \mathrm{GeV}$ we give the values obtained by the fit with (i), since methods (ii) and (iii) should only be applied at higher energies. The results from methods (ii) and (iii) are shown for $E_{\gamma}>1.4 \mathrm{GeV}$. (At higher energies the production angular distribution is peaked towards the forward direction, where the Ross-Stodolsky model should be better applicable.) The difference between the points from methods (ii) and (iii) allows an estimate of the systematic errors for the $\rho$ cross section due to the fact that the form of the background and of the $\pi^{+} \pi^{-}$mass distribution from $\rho$ decay is not precisely known.

Above $2 \mathrm{GeV}$ the $\rho$ cross section is fairly constant with energy. This energy dependence cannot be explained by the one-pion-exchange model but rather indicates a diffractionlike mechanism for $\rho$ production. A fit of the form

$$
\sigma\left(E_{\gamma}\right)=A E_{\gamma}{ }^{-n}
$$

above $2 \mathrm{GeV}$ yielded

$$
\begin{aligned}
A & =18.2 \pm 1.7 \mu \mathrm{b}, \\
n & =0.08 \pm 0.07,
\end{aligned}
$$

where $E_{\gamma}$ is measured in $\mathrm{GeV}$; averages of the points from methods (ii) and (iii) were used.

Figure 9 shows the differential cross section $d \sigma / d \Delta^{2}$ for $\rho$ production for five $E_{\gamma}$ intervals. The corresponding angular distributions in the center-of-mass system are shown in Fig. 10. The numerical values for $d \sigma / d \Delta^{2}$ and $d \sigma / d \Omega$ are listed in Tables III and IV, respectively. The values for $d \sigma / d \Delta^{2}$ were obtained by the fitting method (ii). This method yielded values of $d \sigma / d \Delta^{2}$ somewhat different from our previous results, ${ }^{6}$ which were obtained by method (i). For $d \sigma / d \Omega$ we used method (ii) for $\cos \theta_{\text {c.m. }}>0.7$ only. For $\cos \theta_{\text {c.m. }}<0.7$ we applied method (i), which led to better fits. The errors in the $\rho$ cross sections in Figs. 8-12 are the errors of the parameters fitted by the maximum-likelihood program. Systematic errors due to the uncertainty of the resoance shape used are not included.

The arrows at the most forward point in Figs. 9(c)$9(\mathrm{e})$ and $10(\mathrm{c})-(10 \mathrm{e})$ indicate a possible scanning loss due to very short proton tracks. Evidence that the forward cross section is most likely underestimated in Figs. 9(c)-9(e) and 10(c)-10(e) comes from a sparkchamber experiment. ${ }^{42}$ This experiment shows that $d \sigma / d \Delta^{2}$ can be fitted by an exponential (see below) down

${ }^{42}$ H. Blechschmidt, J. P. Dowd, B. Elsner, K. Heinloth, K. H. Höhne, S. Raither, J. Rathje, D. Schmidt, J. H. Smith, and J. H. Weber, Nuovo Cimento 52A, 1348 (1967). 


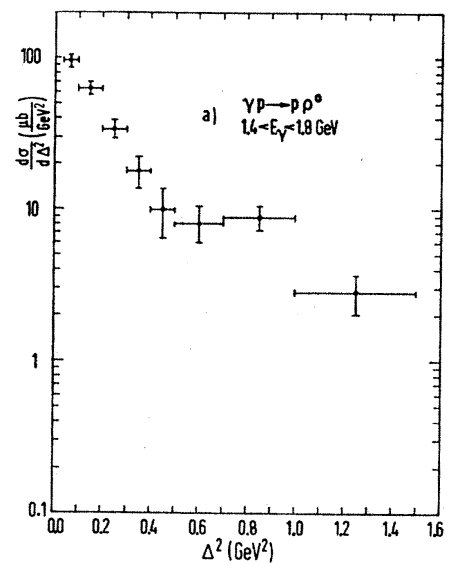

(a)

FIG. 9. Reaction $\gamma p \rightarrow p \rho^{0}$. Differential cross sections $d \sigma / d \Delta^{2}$ for five photon energy intervals. $\Delta^{2}$ is the square of the four-momentum transfer between incoming and outgoing proton. The arrows near the forward direction indicate possible scanning losses.

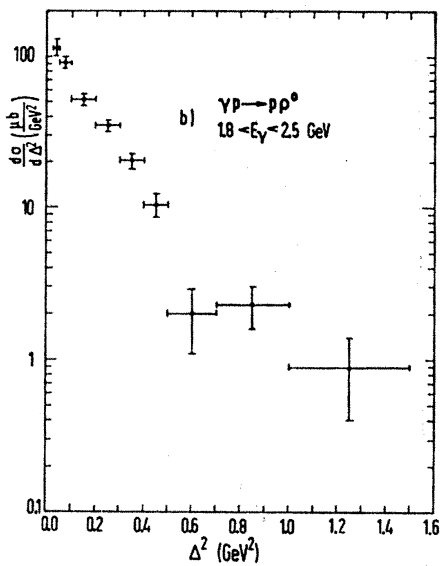

(b)

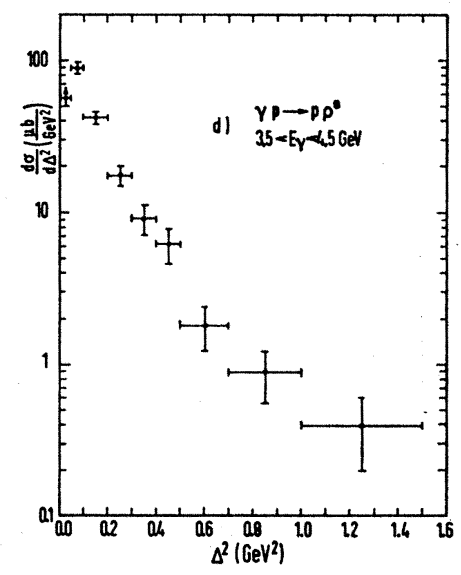

(d)

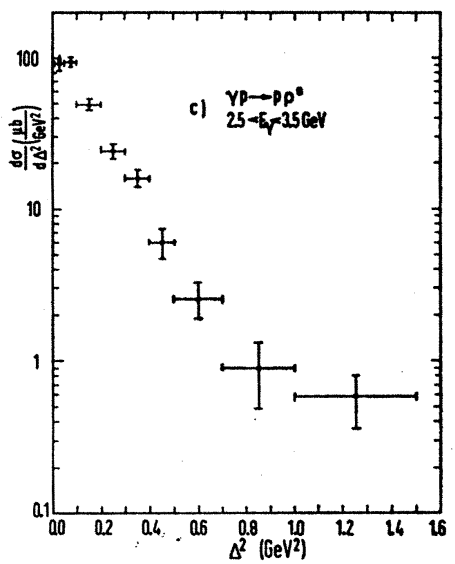

(c)

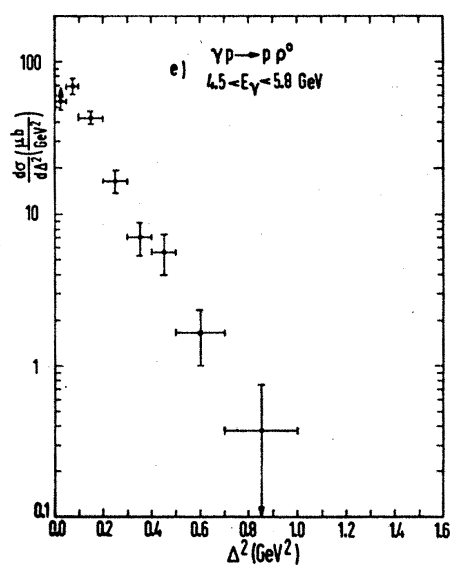

(e) to the smallest values of $\Delta^{2}$ kinematically allowed. The total cross sections shown in Figs. 4 and 8 and the numbers of events in column B of Table $I$ have been corrected accordingly. ${ }^{43}$ The correction has not been applied in our previous publications on $\rho$ meson production. ${ }^{2,3,6}$
The differential cross section $d \sigma / d \Delta^{2}$ (Fig. 9) was fitted to an exponential of the form

$$
d \sigma / d \Delta^{2}=A \exp \left(-B \Delta^{2}\right)
$$

for $0.05 \mathrm{GeV}^{2}<\Delta^{2}<0.5 \mathrm{GeV}^{2}$. The values for $A$ and $B$ resulting from this fit are given in Table $\mathrm{V}$ for five $E_{\gamma}$

TABLE III. Reaction $\gamma p \rightarrow p \rho^{0}$. Differential cross sections $d \sigma / d \Delta^{2}\left(\mu \mathrm{b} / \mathrm{GeV}^{2}\right)$ for five intervals of the photon energy $E_{\gamma}$.

\begin{tabular}{|c|c|c|c|c|c|}
\hline$\underset{\left(\mathrm{GeV}^{2}\right)}{\stackrel{\Delta^{2}}{E_{\gamma}}} E_{(\mathrm{GeV})}$ & $1.4-1.8$ & $1.8-2.5$ & $2.5-3.5$ & $3.5-4.5$ & $4.5-5.8$ \\
\hline$\Delta_{\min ^{2}}-0.05$ & $\ldots$ & $116.0 \pm 14.0$ & $92.0 \pm 8.4^{\mathrm{s}}$ & $57.6 \pm 8.7^{\mathrm{a}}$ & $55.2 \pm 7.1$ \\
\hline $0.05-0.1$ & $97.3 \pm 10.3^{b}$ & $91.4 \pm 7.5$ & $94.8 \pm 7.0$ & $88.6 \pm 7.9$ & $69.6 \pm 7.5$ \\
\hline $0.1-0.2$ & $62.7 \pm 6.4$ & $52.1 \pm 4.2$ & $49.2 \pm 3.6$ & $41.7 \pm 3.9$ & $42.7 \pm 4.1$ \\
\hline $0.2-0.3$ & $33.9 \pm 4.7$ & $35.0 \pm 3.1$ & $24.0 \pm 2.7$ & $17.4 \pm 2.6$ & $16.5 \pm 2.6$ \\
\hline $0.3-0.4$ & $17.7 \pm 4.2$ & $20.3 \pm 2.5$ & $16.0 \pm 2.0$ & $9.2 \pm 2.0$ & $7.1 \pm 1.7$ \\
\hline $0.4-0.5$ & $9.9 \pm 3.5$ & $10.5 \pm 1.8$ & $6.0 \pm 1.4$ & $6.2 \pm 1.6$ & $5.6 \pm 1.7$ \\
\hline $0.5-0.7$ & $8.1 \pm 2.1$ & $2.0 \pm 0.9$ & $2.6 \pm 0.7$ & $1.81 \pm 0.58$ & $1.68 \pm 0.65$ \\
\hline $0.7-1.0$ & $8.8 \pm 1.6$ & $2.3 \pm 0.7$ & $0.90 \pm 0.41$ & $0.88 \pm 0.33$ & $0.37 \pm 0.37$ \\
\hline $1.0-1.5$ & $2.8 \pm 0.8$ & $0.9 \pm 0.5$ & $0.58 \pm 0.22$ & $0.40 \pm 0.20$ & $\cdots$ \\
\hline$\Delta_{\min }^{2}\left(\mathrm{GeV}^{2}\right)$ & & 0.025 & 0.01 & 0.005 & 0.0025 \\
\hline
\end{tabular}

a These values are not corrected for scanning losses due to very short proton tracks. b Calculated from $0.04 \mathrm{GeV}^{2}<\Delta^{2}<0.1 \mathrm{GeV}^{2}$.

${ }^{43}$ Below $E_{\gamma}=2.5 \mathrm{GeV}$ the scanning losses are small, since most of the recoil proton tracks are visible because of the minimum momentum transfer. 


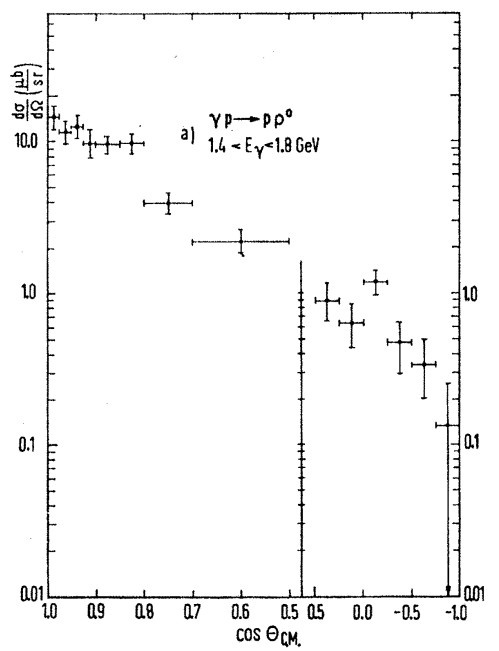

(a)

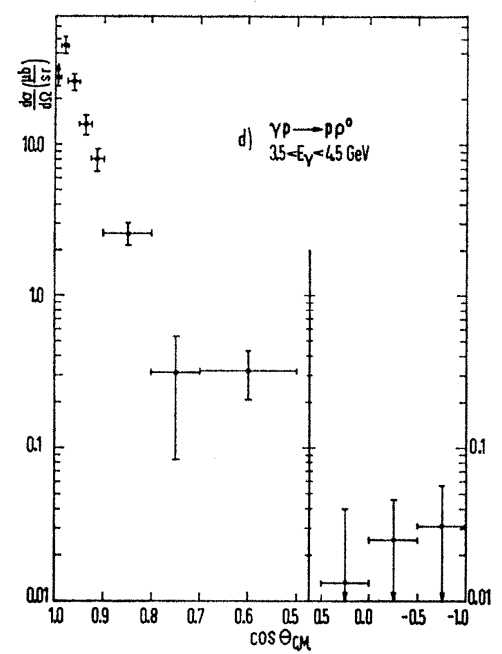

(d)

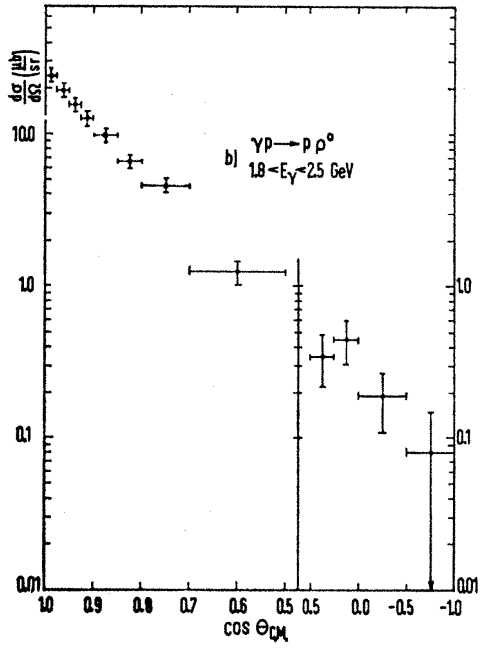

(b)

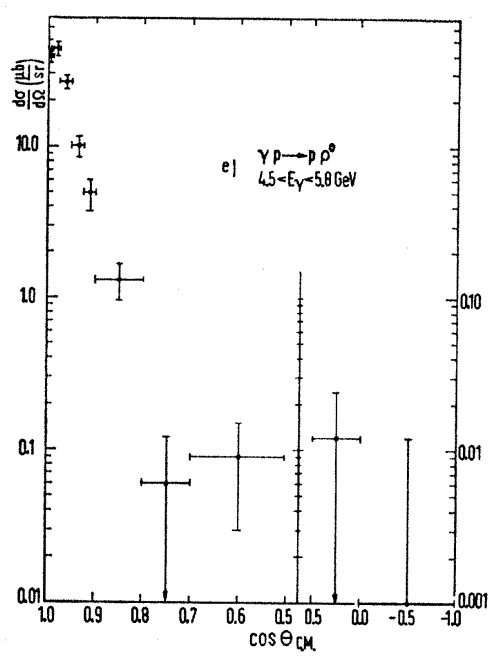

(e)

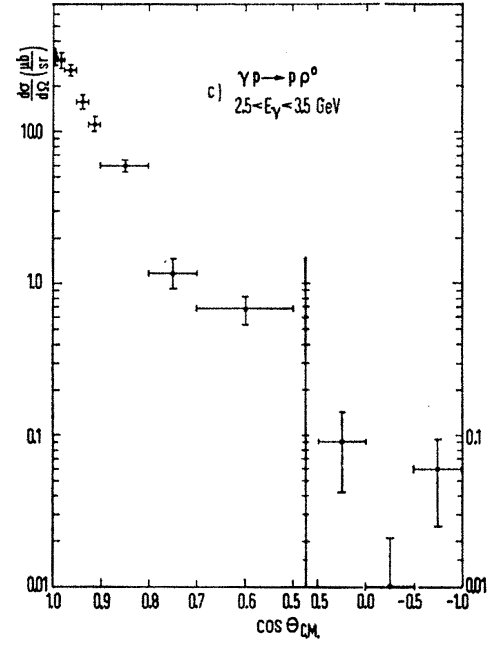

(c)

FIG. 10. Reaction $\gamma p \rightarrow p \rho^{0}$. Differential cross sections $d \sigma / d \Omega$ for five photon-energy intervals; $\theta_{\text {e.m. }}$ is the angle between ingoing and outgoing proton in the over-all center-of-mass system. The arrows indicate possible scanning losses.

intervals. $B$ seems to increase with increasing $E_{\gamma}$. At beam energies of 4-5 GeV the values of the slope $B$ have the same magnitude as those measured for elastic $\pi p$ scattering. It must be noted that the values for the slope $B$ are averages over the whole $\rho$ region. Actually we find a strong variation of $B$ with the $\pi^{+} \pi^{-}$mass. This can be seen in Fig. 11, which shows $d \sigma / d \Delta^{2}$ for six intervals of $M\left(\pi^{+} \pi^{-}\right)$and $E_{\gamma}>2.5 \mathrm{GeV}$. The results of a fit with Eq. (8) to these distributions are given in Table VI. The observed dependence of the slope $B$ on the $\pi^{+} \pi^{-}$mass is not unexpected if one assumes that a diffractive $\rho$-production amplitude interferes with a slowly varying background amplitude. ${ }^{39,40}$ Approximate calculations of this effect predict variations of $B$ quite similar in magnitude to those observed. The dependence of the slope on the $\pi^{+} \pi^{-}$mass is connected with an increase of the observed $\rho$ mass with increasing $\Delta^{2}$.

The differential cross sections show diffractionlike peaking in the forward direction. In order to see whether possible $s$-channel effects (e.g., intermediate isobar formation) contribute to $\rho$ production we have plotted, in Fig. 12, the $\rho$-production cross section as a function of $E_{\gamma}$ for $\cos \theta_{\text {c.m. }}<0.7$, i.e., for production angles outside the diffraction peak. In Fig. 12 there is an enhancement of two standard deviations at the position of the $\Delta(2420)$. Below $E_{\gamma}=2 \mathrm{GeV}$ the corresponding $\rho$ production cross section for $-1<\cos \theta_{\text {c.m. }}<0$ shows no significant structure.

\section{Decay Distributions}

We now discuss the $\rho$ decay distribution in three different coordinate systems. The three systems differ in the choice of the $z$ axis. In the Jackson system the $z$ direction is chosen as the direction of the incident photon in the $\pi^{+} \pi^{-}$c.m. system. In the helicity system the $z$ direction is chosen opposite to the direction of the outgoing proton in the $\pi^{+} \pi^{-}$c.m. system (i.e., equal to the direction of flight of the $\rho$ meson in the over-all 
TABLE IV. Reaction $\gamma p \rightarrow p \rho^{0}$. Differential cross sections $d \sigma / d \Omega(\mu \mathrm{b} / \mathrm{sr})$ for five intervals of the photon energy $E_{\gamma}$.

\begin{tabular}{|c|c|c|c|c|}
\hline \multicolumn{2}{|c|}{$\cos \theta_{\text {c.m. }}$} & \multicolumn{2}{|c|}{$1.4 \mathrm{GeV}<E_{\gamma}<1.8 \mathrm{GeV}$} & $1.8 \mathrm{GeV}<E_{\gamma}<2.5 \mathrm{GeV}$ \\
\hline \multicolumn{2}{|c|}{$\begin{array}{l}0.975-1.0 \\
0.95-0.975 \\
0.925-0.95 \\
0.9-0.925 \\
0.85-0.9 \\
0.8-0.85 \\
0.7-0.8 \\
0.5-0.7 \\
0.25-0.5 \\
0.0-0.25 \\
(-0.25)-0.0 \\
(-0.5)-(-0.25) \\
(-0.75)-(-0.5) \\
(-1.0)-(-0.75)\end{array}$} & \multicolumn{2}{|c|}{$\begin{array}{l}14.5 \pm 2.5 \\
11.8 \pm 2.1 \\
12.6 \pm 2.1 \\
9.8 \pm 2.0 \\
9.55 \pm 1.25 \\
9.68 \pm 1.36 \\
3.92 \pm 0.63 \\
2.19 \pm 0.35 \\
0.89 \pm 0.24 \\
0.64 \pm 0.20 \\
1.18 \pm 0.22 \\
0.47 \pm 0.17 \\
0.34 \pm 0.15 \\
0.13 \pm 0.13\end{array}$} & $\begin{array}{c}24.1 \pm 2.1 \\
19.5 \pm 1.8 \\
15.7 \pm 1.6 \\
12.7 \pm 1.4 \\
9.74 \pm 0.97 \\
6.55 \pm 0.73 \\
4.55 \pm 0.47 \\
1.24 \pm 0.21 \\
0.35 \pm 0.13 \\
0.45 \pm 0.14 \\
0.19 \pm 0.08 \\
\{0.08 \pm 0.06\end{array}$ \\
\hline \multicolumn{2}{|c|}{$\cos \theta_{\text {c.m. }}$} & $2.5 \mathrm{GeV}<E_{\gamma}<3.5 \mathrm{GeV}$ & $3.5 \mathrm{GeV}<E_{\gamma}<4.5 \mathrm{GeV}$ & $4.5 \mathrm{GeV}<E_{\gamma}<5.8 \mathrm{GeV}$ \\
\hline $\begin{array}{r}0.9875- \\
0.975= \\
0.95= \\
0.925= \\
0.9= \\
0.8= \\
0.7= \\
0.5= \\
0.0= \\
(-0.5)= \\
(-1.0)-(-\end{array}$ & $\begin{array}{l}1.0 \\
0.9875 \\
0.975 \\
0.95 \\
0.925 \\
0.9 \\
0.8 \\
0.7 \\
0.5 \\
0.0 \\
-0.5)\end{array}$ & $\begin{array}{c}30.4 \pm 3.1 \mathrm{a} \\
29.5 \pm 3.7 \\
25.5 \pm 2.0 \\
15.7 \pm 1.7 \\
11.2 \pm 1.4 \\
5.9 \pm 0.5 \\
1.18 \pm 0.27 \\
0.67 \pm 0.14 \\
0.09 \pm 0.05 \\
0.0 \pm 0.02 \\
0.06 \pm 0.03\end{array}$ & $\begin{array}{cl}27.6 & \pm 3.6 \mathrm{a} \\
45.2 & \pm 5.5 \\
26.0 & \pm 3.1 \\
13.6 & \pm 1.8 \\
8.0 & \pm 1.4 \\
2.56 & \pm 0.41 \\
0.31 & \pm 0.23 \\
0.32 & \pm 0.11 \\
0.013 \pm 0.026 \\
0.025 \pm 0.021 \\
0.031 \pm 0.026\end{array}$ & $\begin{array}{cl}39.5 & \pm 4.4 \mathrm{a} \\
43.9 \pm 4.7 & \\
26.3 & \pm 2.6 \\
10.1 & \pm 1.6 \\
4.9 & \pm 1.2 \\
1.30 \pm 0.35 \\
0.06 \pm 0.06 \\
0.36 \pm 0.26 \\
0.09 \pm 0.06 \\
0.012 \pm 0.012 \\
0.0 \pm 0.012\end{array}$ \\
\hline
\end{tabular}

a These values are not corrected for scanning losses due to very short proton tracks.

c.m. system). In the Adair system the $z$ direction is chosen as the direction of the photon momentum in the over-all c.m. system.

The $y$ direction is the normal to the production plane, defined by the cross product $\mathbf{p}_{\text {in }} \times \mathbf{p}_{\text {out }}$ of the momenta of incoming and outgoing protons. The $x$ direction is given by $\mathbf{x}=\mathbf{y} \times \mathbf{z}$. The decay angles $\theta$ and $\phi$ are defined in the desired coordinate system as follows: $\theta$ is the angle between the $z$ direction and the outgoing $\pi^{+}$in the $\pi^{+} \pi^{-}$c.m. system. $\phi$ is the corresponding azimuth angle with $\phi=0$ in the production plane ( $x z$ plane);

$$
\begin{aligned}
& \cos \theta=\boldsymbol{\pi}^{+} \cdot \mathbf{z}, \\
& \cos \phi=\mathbf{y} \cdot\left(\mathbf{z} \times \boldsymbol{\pi}^{+}\right) /\left|\mathbf{z} \times \boldsymbol{\pi}^{+}\right|, \\
& \sin \phi=-\mathbf{x} \cdot\left(\mathbf{z} \times \boldsymbol{\pi}^{+}\right) /\left|\mathbf{z} \times \boldsymbol{\pi}^{+}\right|,
\end{aligned}
$$

where $\mathbf{x}, \mathbf{y}, \mathbf{z}$ are the unit vectors of the coordinate system, and $\pi^{+}$is a unit vector in the direction of flight of the $\pi^{+}$in the $\pi^{+} \pi^{-}$c.m. system. For the three systems the angles are labeled with subscripts as $\theta_{J}, \phi_{J}$, $\theta_{H}, \phi_{H}$, and $\theta_{A}, \phi_{A}$, respectively.

TABLE V. Reaction $\gamma p \rightarrow p \rho^{0}$. Fit of $A \exp \left(-B \Delta^{2}\right)$ to the differential cross section $d \sigma / d \Delta^{2}$ for $0.05 \mathrm{GeV}^{2}<\Delta^{2}<0.5 \mathrm{GeV}^{2}$.

\begin{tabular}{ccc}
\hline \hline $\begin{array}{c}E_{\gamma} \\
(\mathrm{GeV})\end{array}$ & $\begin{array}{c}A \\
\left(\mu \mathrm{b} / \mathrm{GeV}^{2}\right)\end{array}$ & $\begin{array}{c}B \\
\left(\mathrm{GeV}^{-2}\right)\end{array}$ \\
\hline $1.4-1.8$ & $140.7 \pm 19.0$ & $5.75 \pm 0.65$ \\
$1.8-2.5$ & $128.8 \pm 11.3$ & $5.43 \pm 0.39$ \\
$2.5-3.5$ & $146.8 \pm 13.0$ & $6.92 \pm 0.43$ \\
$3.5-4.5$ & $149.3 \pm 18.5$ & $8.10 \pm 0.69$ \\
$4.5-5.8$ & $129.7 \pm 16.2$ & $7.90 \pm 0.66$ \\
\hline \hline
\end{tabular}

Figure 13 shows distributions of the $\rho$-decay angle $\cos \theta_{H}$ for two intervals of the production angle and the photon energy $E_{\gamma}$. It is seen that for small production angles the decay distribution is compatible with $\sin ^{2} \theta_{H}$, which is expected for complete spin alignment of the $\rho$ meson along its direction of flight (helicity $= \pm 1$ ). For larger production angles this is no longer the case.

Figure 14 shows the forward-backward asymmetry $R=(F-B) /(F+B)$ for all events of reaction (1a) as functions of the $\pi^{+} \pi^{-}$mass for five $E_{\gamma}$ intervals. $F$ and $B$ are the numbers of events with $\cos \theta_{J}>0$ and $\cos \theta_{J}<0$, respectively. In the $\rho$ meson mass region, $R$ is consistent

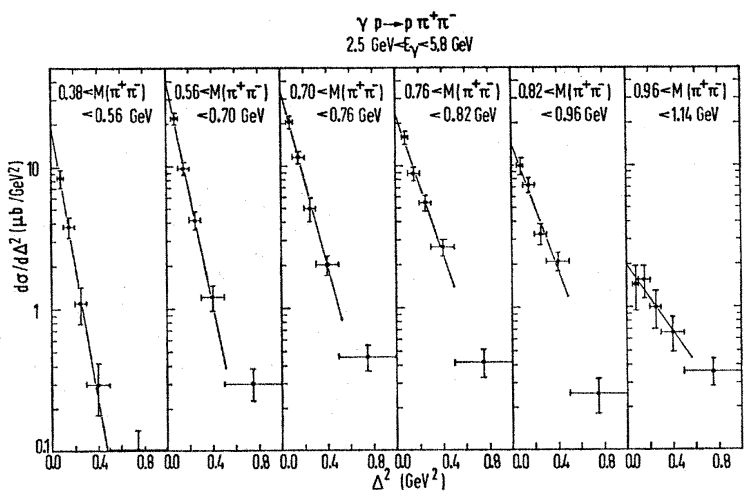

Fig. 11. Reaction $\gamma p \rightarrow p \pi^{+} \pi^{-}$at photon energies $2.5<E_{\gamma}<5.8$ $\mathrm{GeV}$. Differential cross section $d \sigma / d \Delta^{2}$ for six intervals of $M\left(\pi^{+} \pi^{-}\right)$. The points contain all events of reaction $\gamma p \rightarrow p \pi^{+} \pi^{-}$which do not form a $\Delta^{++}$. The respective numbers of $\Delta^{++}$events as determined by fits to the corresponding mass distributions $M\left(p \pi^{+}\right)$were subtracted. The curves are fits by an exponential $A \exp \left(-B \Delta^{2}\right)$ for $0.05<\Delta^{2}<0.5 \mathrm{GeV}^{2}$. 


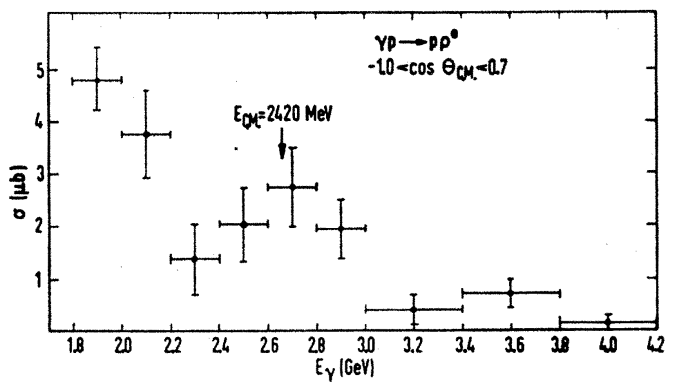

FIG. 12. Reaction $\gamma p \rightarrow p \rho^{0}$. Cross section for $-1.0<\cos \theta_{\text {c.m. }}$. $<0.7$ as a function of the photon energy $E_{\gamma} . E_{\text {e.m. }}$ is the total c.m. energy.

with zero. This means that there is no indication for significant production of even-parity dipion states at masses in the neighborhood of the $\rho$ resonance. The corresponding data on $\pi^{-} p \rightarrow \pi^{+} \pi^{-} n$, on the contrary, show strong forward-backward asymmetries, which are interpreted as being due to a strong (possibly resonant) $I=0, J^{P}=0^{+}$pion-pion partial wave and to some $I=0$, $J^{P}=2^{+}$contribution (tail of the $f$ resonance). In our experiment $f^{0}$ production is very weak; see Sec. VII.

The decay angular distribution $W(\cos \theta, \phi)$ is conveniently expressed by the spin-space density matrix elements $\rho_{i k}$ of the $\rho$ meson ${ }^{44}$

$$
\begin{aligned}
W(\cos \theta, \phi) & =(3 / 4 \pi)\left[\frac{1}{2}\left(1-\rho_{00}\right)+\frac{1}{2}\left(3 \rho_{00}-1\right) \cos ^{2} \theta\right. \\
& \left.-\rho_{1-1} \sin ^{2} \theta \cos 2 \phi-\sqrt{2} \operatorname{Re} \rho_{10} \sin 2 \theta \cos \phi\right] .
\end{aligned}
$$

This formula holds for all three coordinate systems defined above. The matrix elements $\rho_{00}, \rho_{1-1}$, and $\operatorname{Re} \rho_{10}$ for the $\rho$ meson were determined in all three coordinate systems by maximum-likelihood fits to the data. The fitting procedure is described in the Appendix. The results are listed in Table VII for various intervals of the production angle in the c.m. system and for four intervals of $E_{\gamma}$. The results for the matrix elements in the helicity system are also shown in Fig. 15. The errors
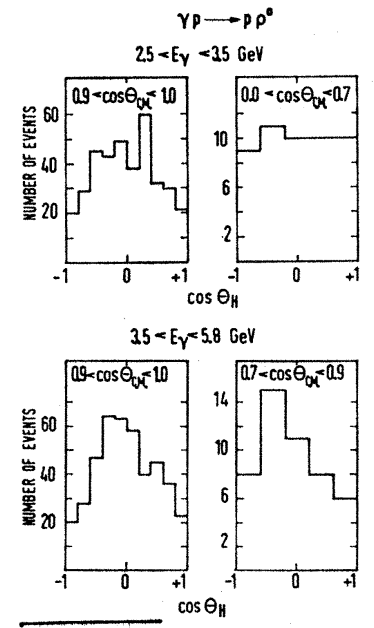

Fig. 13. Reaction $\gamma p \rightarrow p \rho^{0}$. $\rho$ decay distributions $W\left(\cos \theta_{H}\right)$ in the helicity system for two intervals of the c.m. production angle $\cos \theta_{\text {c.m. }}$ at two photon-energy intervals. The distributions were taken from the mass region $0.68<M\left(\pi^{+} \pi^{-}\right)$ $<0.84 \mathrm{GeV}$ without background subtraction.

${ }^{44} \mathrm{~K}$. Gottfried and J. D. Jackson, Nuovo Cimento 33, 309 (1964).

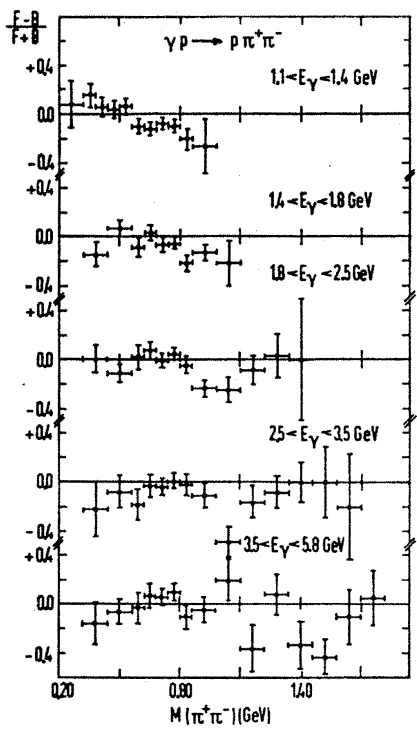

FIG. 14. Reaction $\gamma p \rightarrow p \pi^{+} \pi^{-}$. Forward-backward asymmetry $R=(F-B) /(F+B)$ as a function of the $\pi^{+} \pi^{-}$mass for five intervals of $E_{\gamma} . F$ and $B$ are the numbers of events with $\cos \theta_{J}>0$ and $\cos \theta_{J}<0$, respectively, where $\theta_{J}$ is the $\rho$-decay angle in the Jackson system (see Sec. III C).

given in Fig. 15 and Table VII are purely statistical and do not take into account possible uncertainties in the background subtraction.

For $\cos \theta_{\text {c.m. }} \geq 0.7$ and $E_{\gamma}>1.8 \mathrm{GeV}$, the density matrix elements are seen to be roughly consistent with "conservation of helicity" (i.e., $\rho_{00}{ }^{H}=\rho_{1-1}{ }^{H}=\operatorname{Re} \rho_{10}{ }^{H}=0$ in the helicity system). ${ }^{45}$ For comparison we include in Fig. 15 the predictions following from the assumption $m_{\gamma}=m_{\rho}, m_{p}=m_{p^{\prime}}$, where $m_{\gamma}, m_{\rho}, m_{p}$, and $m_{p^{\prime}}$ are the spin components of the'photon, the $\rho$ meson, the incident proton, and the outgoing proton, respectively, relative to the photon direction in the c.m. system. This assumption has been used in the "strong-absorption model" (SAM) by Eisenberg et al. ${ }^{46}$ In the helicity frame used for Fig. 15, the density matrix elements predicted by

TABLE VI. Reaction $\gamma p \rightarrow p \pi^{+} \pi^{-}$at photon energies 2.5 $\mathrm{GeV}<E_{\gamma}<5.8 \mathrm{GeV}$. Fit of $A \exp \left(-B \Delta^{2}\right)$ to the differential cross sections from Fig. 11 for $0.05 \mathrm{GeV}^{2}<\Delta^{2}<0.5 \mathrm{GeV}^{2}$ as a function of $M\left(\pi^{+} \pi^{-}\right)$.

\begin{tabular}{cc}
\hline \hline $\begin{array}{c}M\left(\pi^{+} \pi^{-}\right) \\
(\mathrm{GeV})\end{array}$ & $\begin{array}{c}B \\
\left(\mathrm{GeV}^{-2)}\right.\end{array}$ \\
\hline $0.38-0.56$ & $10.74 \pm 1.2$ \\
$0.56-0.70$ & $9.06 \pm 0.57$ \\
$0.70-0.76$ & $7.17 \pm 0.47$ \\
$0.76-0.82$ & $5.41 \pm 0.46$ \\
$0.82-0.96$ & $5.01 \pm 0.54$ \\
$0.96-1.14$ & $2.62 \pm 0.95$ \\
\hline
\end{tabular}

45 The density matrix elements in the Jackson, helicity, and Adair system, respectively, are labeled by superscripts $J, H$, and $A$.

${ }^{46}$ Y. Eisenberg, E. E. Ronat, A. Brandstetter, A. Levy, and E. Gotsman, Phys. Letters 22, 217 (1966); 22, 223 (1966). 


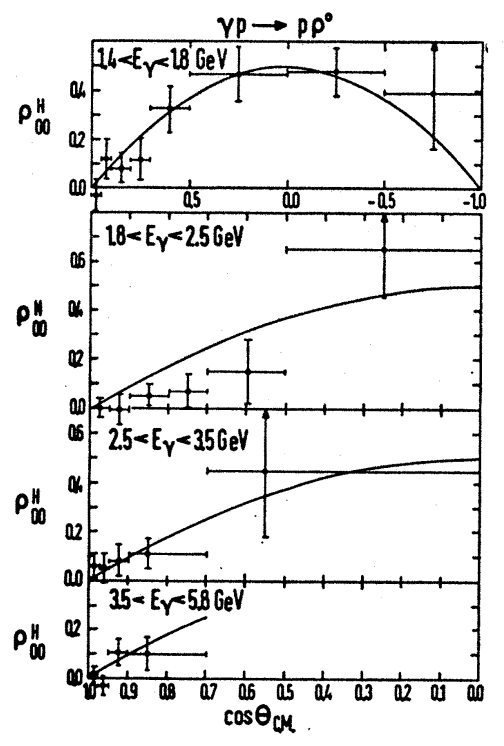

(a)

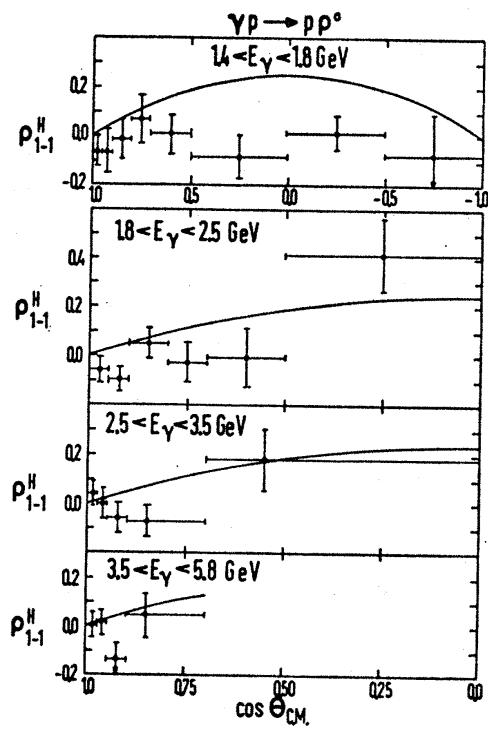

(b)
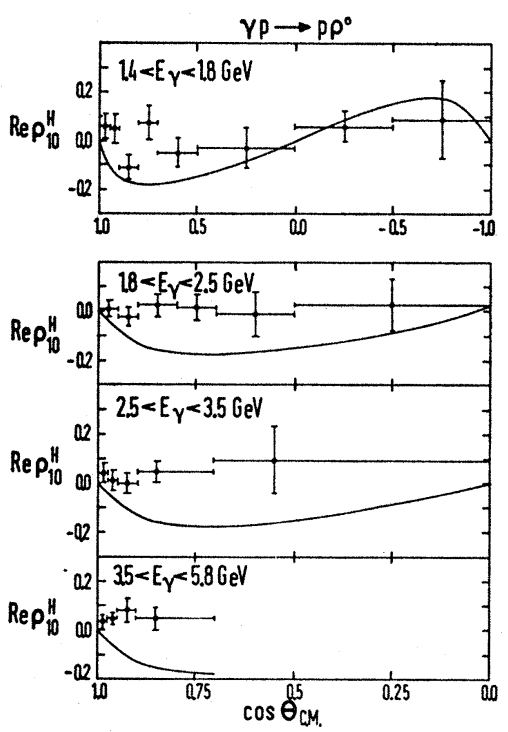

(c)

FIG. 15. Reaction $\gamma p \rightarrow p \rho^{0}$ in four photon-energy intervals. Density matrix elements of the $\rho^{0}$ in the helicity system as functions of the c.m. production angle $\cos \theta_{\text {c.m. }}$., (a) $\rho_{00}{ }^{H}$, (b) $\rho_{1-1}{ }^{B}$, (c) $\operatorname{Re} \rho_{10}{ }^{H}$. The curves are from Ref. 47.

SAM are given by ${ }^{47}$

$$
\begin{aligned}
\rho_{00}{ }^{H} & =\frac{1}{2} \sin ^{2} \theta_{\text {c.m. }}, \\
\rho_{1-1}{ }^{H} & =\frac{1}{4} \sin ^{2} \theta_{\text {c.m. }}, \\
\operatorname{Re} \rho_{10}{ }^{H} & =(-1 / 4 \sqrt{2}) \sin 2 \theta_{\text {c.m. }} .
\end{aligned}
$$

In the case of $\rho_{00}{ }^{H}$ one finds reasonable agreement, whereas for $\rho_{1-1}{ }^{H}$ and $\operatorname{Re} \rho_{10}{ }^{H}$ there are discrepancies. In the Adair system, the above assumption amounts simply to $\rho_{00}{ }^{A}=\rho_{1-1}{ }^{A}=\operatorname{Re} \rho_{10}{ }^{A}=0 .{ }^{47}$ This is, in general, not true for the data, as can be seen from Table VII.

We have looked for a dependence of the density matrix elements on the $\pi \pi$ mass. Such a dependence is expected if one assumes that a diffractive $\rho$ amplitude interferes with a Drell-type background. ${ }^{48}$ The experimental matrix elements ${ }^{49}$ are compatible with independence of the $\pi^{+} \pi^{-}$mass. But the errors are much larger than the effects predicted in Ref. 48.

\section{Summary}

The photoproduction of $\rho$ mesons in the reaction $\gamma p \rightarrow p \rho^{0}$ shows a diffractionlike behavior. The total cross section above $E_{\gamma}=2 \mathrm{GeV}$ is approximately constant. It can be described by $\sigma\left(E_{\gamma}\right)=18.2 E_{\gamma}{ }^{-0.08}$ (where $\sigma$ is measured in $\mu \mathrm{b}$ and $E_{\gamma}$ in $\mathrm{GeV}$ ). The differential cross sections $d \sigma / d \Delta^{2}$ at $E_{\gamma}=4-5 \mathrm{GeV}$ have the same slope of about $8 \mathrm{GeV}^{-2}$ as in elastic $\pi p$ scattering. The region below $E_{\gamma}=2 \mathrm{GeV}$ is not well understood. The determination of total cross sections suffers from the lack of knowledge of the exact $\rho$ resonant

${ }^{47}$ G. Kramer, DESY Report No. 67/32, 1967 (unpublished).

48 A. S. Krass, Phys. Rev. 159, 1496 (1967).

${ }^{49}$ Here, the density matrix elements have been determined from all events after $\Delta^{++}$subtraction. shape and of the influence of background processes. The data indicate a weak $s$-channel production of $\gamma p \rightarrow \Delta(2420) \rightarrow p \rho^{0}$. The experimental density matrix elements have been compared with two assumptions
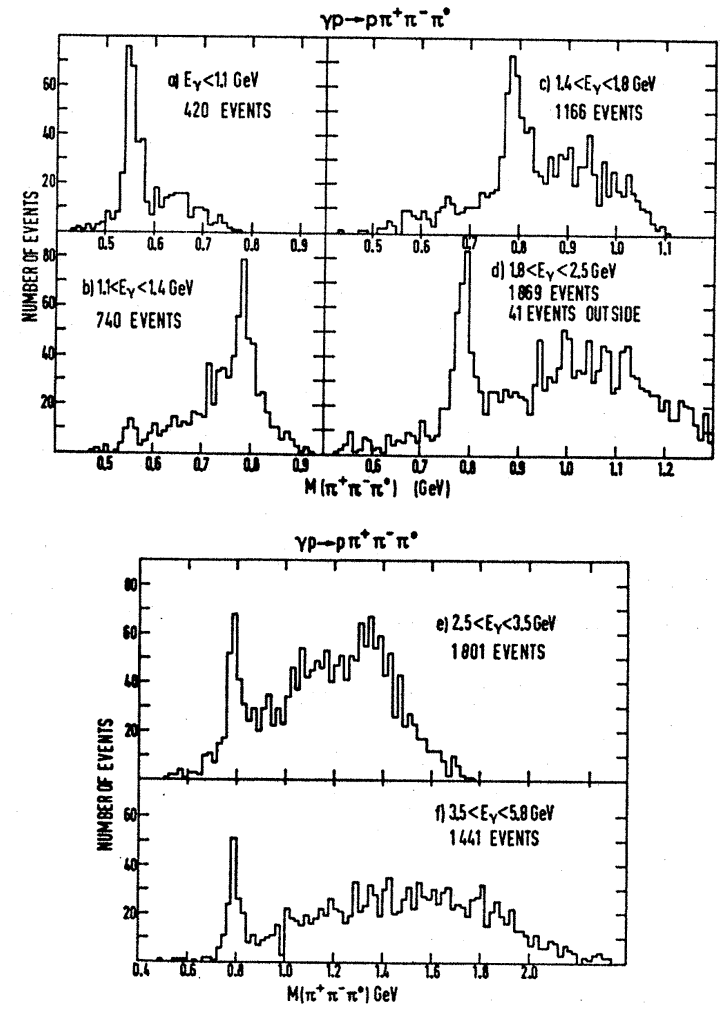

FIG. 16. Effective-mass distributions $M\left(\pi^{+} \pi^{-} \pi^{0}\right)$ for all events compatible with hypothesis $\gamma p \rightarrow p \pi^{+} \pi^{-} \pi^{0}$ for six intervals of $E_{\gamma}$. 
TABLE VII. Reaction $\gamma p \rightarrow \not \rho^{0}$. Density matrix elements of the $\rho^{0}$ in three different reference systems.

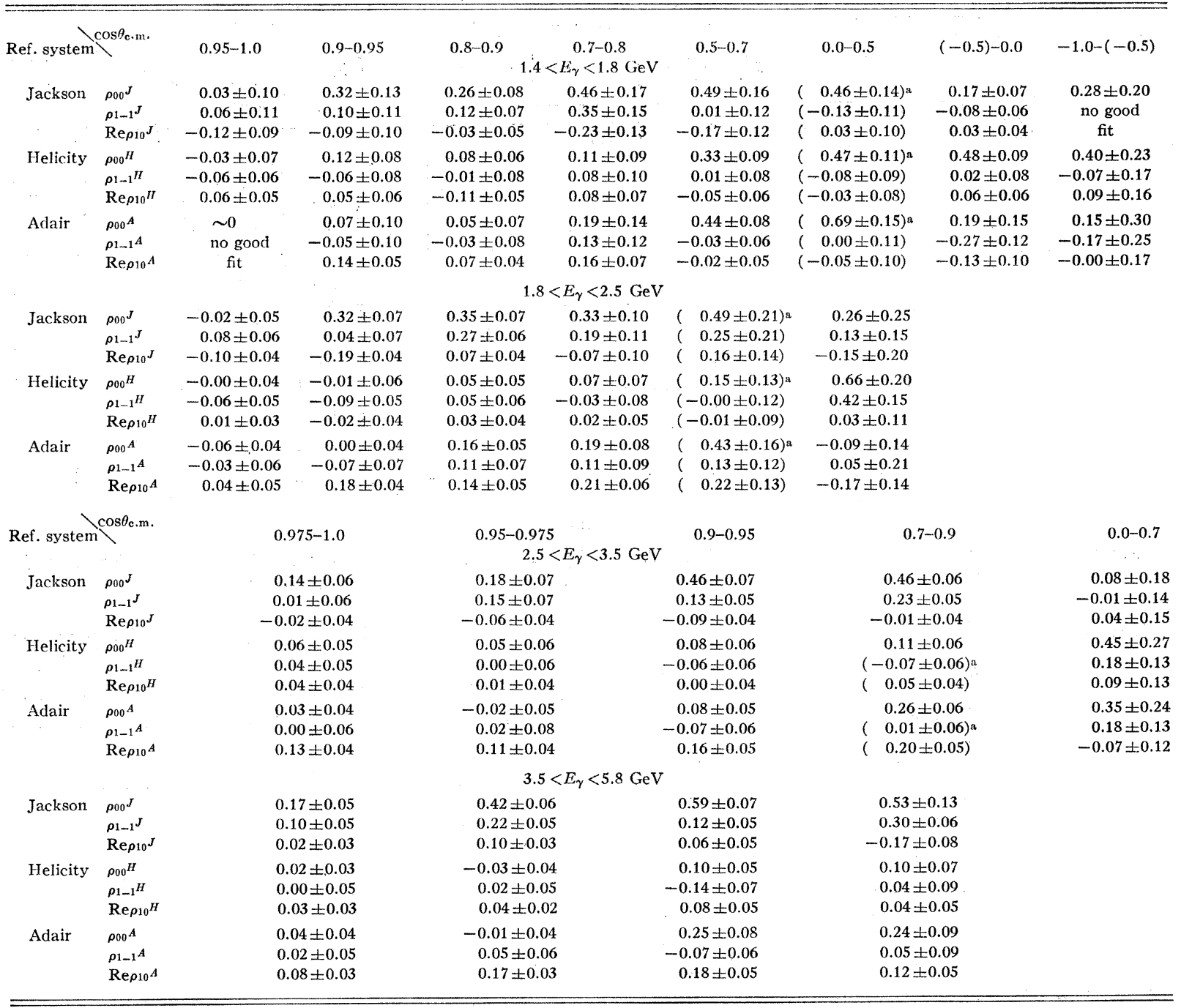

a The decay distribution is asymmetric.

on the spin dependence of $\rho$ production: (a) "spin independence" (i.e., $m_{\gamma}=m_{\rho}$, where $m$ denotes the spin components along the $\gamma$ direction in the c.m. system) and (b) "helicity conservation" $\left(\lambda_{\gamma}=\lambda_{\rho}\right.$, where $\lambda$ denotes the helicities). No over-all agreement is found. The latter assumption is favored by our data.

\section{THE REACTION $\gamma p \rightarrow p \omega$}

Photoproduction of the $\omega$ meson

$$
\gamma p \rightarrow p \omega
$$

was studied in the reaction

$$
\gamma p \rightarrow p \pi^{+} \pi^{-} \pi^{0}
$$

Figure 16 shows the $\pi^{+} \pi^{-} \pi^{0}$ effective-mass distributions for six $E_{\gamma}$ intervals. One observes clear evidence for $\eta$ and $\omega$ production. Figure 17 shows the cross section for $\omega$ production in reaction (2b) as a function of $E_{\gamma}$. The cross sections were obtained by counting the events in the resonance peak. The fraction of events in the tails outside the peak, which are lost by this method, has been determined by FAKE calculations and has been appropriately corrected $(10-15 \%)$. The errors of the points include an estimated uncertainty due to background subtraction. The observed cross sections have been multiplied by a factor $1 / 0.90$ to account for the unobserved decay modes of the $\omega^{41}$ In addition, a correction was applied for scanning losses due to short proton tracks in a similar way as for the $\rho$ cross section.

Above $2.1 \mathrm{GeV}$ the total cross section was fitted to a sum of two terms of the form

$$
\sigma=A E_{\gamma}^{-1.6}+B E_{\gamma}^{-0.08}
$$

The first term describes the typical energy dependence of processes which are believed to go via one-meson 
exchange. The exponent -1.6 is taken as an average from the compilation of Morrison. ${ }^{50}$ The second term has the energy dependence of diffractive processes. The exponent -0.08 was taken from the energy dependence of $\rho$ production (see Sec. III). The result of the fit is

$$
\begin{aligned}
& A=18.4 \pm 5.8 \mu \mathrm{b}, \\
& B=1.9 \pm 0.9 \mu \mathrm{b},
\end{aligned}
$$

where $E_{\gamma}$ is measured in $\mathrm{GeV}$.

An analysis more refined than the one presented above has been made in Ref. 51. It is based on the assumption that the $\omega$ production proceeds by both diffraction and one-pion exchange (OPE), the latter being modified by initial- and final-state absorption. The contribution of OPE is determined from the energy dependence of the total cross section. This analysis leads to an $\omega$ decay width $\Gamma(\omega \rightarrow \pi \gamma)=0.7 \pm 0.2$ $\mathrm{MeV}$ (most probable value).

Figures 18 and 19 show the differential cross section $d \sigma / d \Delta^{2}$ and $d \sigma / d \Omega$ (in the c.m. system), respectively, for various $E_{\gamma}$ intervals. The distributions of Fig. 18 have been fitted to an exponential of the form of Eq. (8). The results of the fit and the range of $\Delta^{2}$ used are given in Table VIII.

Figures 20-22 show for two $E_{\gamma}$ intervals the experimental values for the spin density matrix elements of the $\omega$ as functions of the c.m. production angle $\theta_{\text {c.m. }}$ in the Jackson, helicity, and Adair system. The decay angles of the $\omega$ are defined in the same way as for the $\rho$ if one substitutes the direction of the $\pi^{+}$from the $\rho$ decay by the normal to the $\omega$ decay plane (given by the vector product $\left.\pi^{+} \times \pi^{-}\right)$. The decay angular distribution is given by Eq. (9). The fitting procedure is described in Appendix B.

\section{REACTION $\gamma p \rightarrow p \phi$}

For all three-prong events we have tried a kinematical fit to the hypothesis

$$
\gamma p \rightarrow p K^{+} K^{-} .
$$

Figure 23(a) shows the $K^{+} K^{-}$mass distributions for all events which gave a good fit for this hypothesis and could not be excluded on the basis of ionization. The distribution shows evidence for $\phi$ production. Figure 23(b) shows $\phi$ production in the $K^{0} \bar{K}^{0}$ mass distribution from all events which were compatible with the hypothesis

$$
\gamma p \rightarrow p K^{0} \bar{K}^{0}
$$

with at least one visible $K^{0}$ decay in the chamber. After correction for the neutral decay mode of the $K_{s}{ }^{0}$ and for decays outside the chamber, the numbers of events in the $\phi$ region of the $K^{+} K^{-}$and $K^{0} \bar{K}^{0}$ mass distribution for photon energies below $3.5 \mathrm{GeV}$ agree with

${ }^{50}$ D. R. O. Morrison, Phys. Letters 22, 528 (1966).

${ }^{51}$ K. Schilling and F. Storim, DESY Report No. 68/23, 1968 (unpublished).

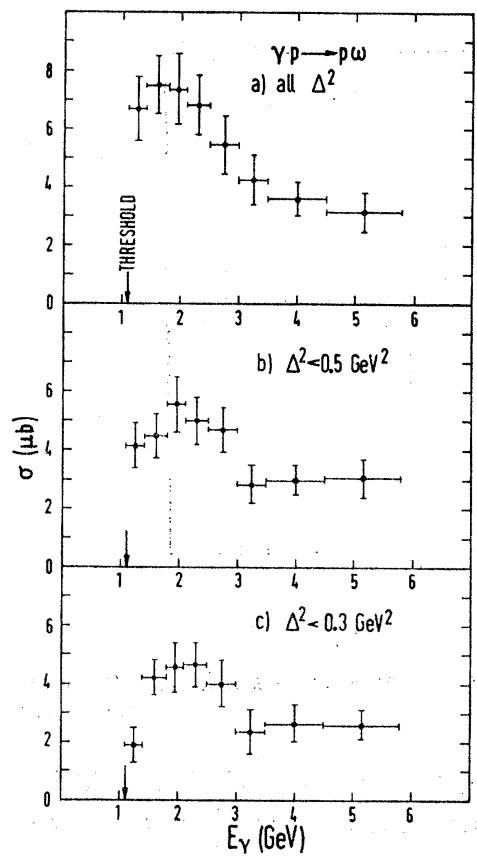

FIG. 17. Cross section for $\gamma p \rightarrow p \omega$ as a function of the photon energy. (a) for all $\Delta^{2}$, (b) for $\Delta^{2}<0.5 \mathrm{GeV}^{2}$, (c) for $\Delta^{2}<0.3 \mathrm{GeV}^{2}$. $\Delta^{2}$ is defined as in the caption of Fig. 9.

the known decay branching ratio of the $\phi^{41}$ For $E_{\gamma}>3.5 \mathrm{GeV}$ some of the $p K^{0} \bar{K}^{0}$ events may have been lost in the scan. Therefore the cross section for $\phi$ production

$$
\gamma p \rightarrow p \phi
$$

has been calculated from events with the charged decay mode $\phi \rightarrow K^{+} K^{-}$alone. The cross section as a function of $E_{\gamma}$ is shown in Fig. 23(c). The cross sections have been corrected by a factor $1 / 0.473$ for the non- $K^{+} K^{-}$ decay modes of the $\phi^{41}$ The cross sections agree with our results from half the statistics. ${ }^{5}$ From a careful reanalysis of the whole sample we have found that the preliminary value for $E_{\gamma}>3.5 \mathrm{GeV}$ given at the Stanford Conference ${ }^{9}$ was too low.

The differential cross sections $d \sigma / d \Delta^{2}$ and $d \sigma / d \Omega$ (in the c.m. system) for $\phi$ production are shown in Figs. 24(a)-24(d) for two $E_{\gamma}$ intervals. The cross section $d \sigma / d \Delta^{2}$ was fitted to an exponential of the form Eq. (8) for $\Delta^{2}<1.0 \mathrm{GeV}^{2}$. The values for $A$ and $B$ re-

TABLE VIII. Reactions $\gamma p \rightarrow p \omega$ and $\gamma p \rightarrow p \phi$. Fit of $A \exp \left(-B \Delta^{2}\right)$ to the differential cross sections $d \sigma / d \Delta^{2}$.

\begin{tabular}{ccccc}
\hline Reaction & $\begin{array}{c}E_{\gamma} \\
(\mathrm{GeV})\end{array}$ & $\begin{array}{c}A \\
\left(\mu \mathrm{b} / \mathrm{GeV}^{2}\right)\end{array}$ & $\begin{array}{c}B \\
\left(\mathrm{GeV}^{-2}\right)\end{array}$ & $\begin{array}{c}\text { Range of } \\
\Delta^{2} \text { used } \\
\left(\mathrm{GeV}^{2}\right)\end{array}$ \\
\hline \multirow{3}{*}{$\gamma p \rightarrow p \omega^{0}$} & $1.4-1.8$ & $43.9 \pm 12.6$ & $6.3 \pm 1.4$ & $0.04-0.5$ \\
& $1.8-2.5$ & $37.6 \pm 8.1$ & $5.4 \pm 1.0$ & $0.02-0.5$ \\
& $2.5-5.8$ & $28.4 \pm 5.2$ & $7.6 \pm 1.2$ & $0.02-0.5$ \\
$\gamma p \rightarrow p \phi^{0}$ & $1.58-2.5$ & $1.13 \pm 0.7$ & $2.7 \pm 1.1$ & $0.1-1.0$ \\
& $2.5-5.8$ & $1.6 \pm 0.6$ & $3.5 \pm 0.9$ & $0.026-1.0$ \\
\hline
\end{tabular}




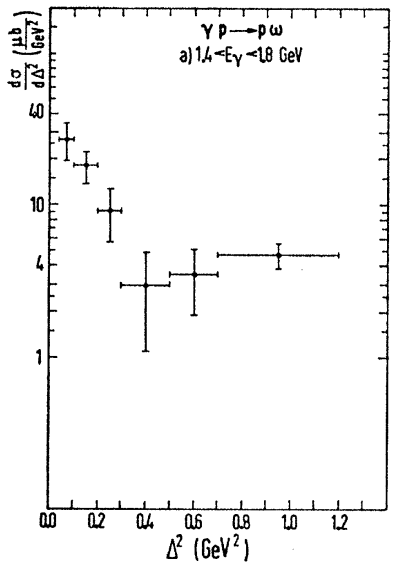

(a)

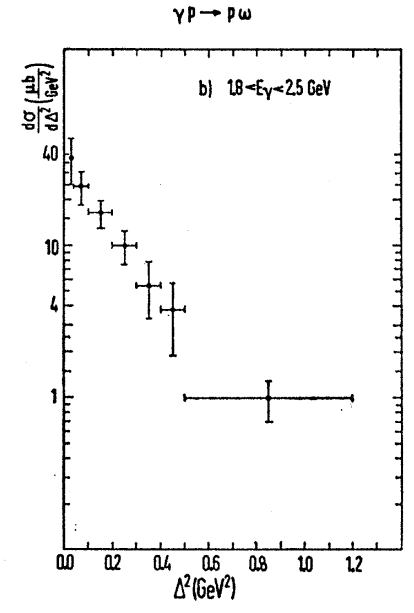

(b)

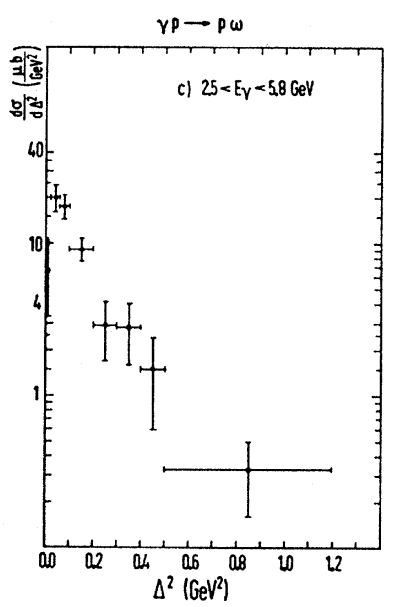

(c)

FIG. 18. Reaction $\gamma p \rightarrow p \omega$. Differential cross sections $d \sigma / d \Delta^{2}$ for three photon-energy intervals. The arrow in Fig. 18(c) indicates possible scanning losses due to short proton tracks.

sulting from this fit are included in Table VIII. The slope $B$ comes out much smaller than for $\rho$ and $\omega$ production. However, it should be kept in mind that the slope was fitted at values of $\Delta^{2}$ up to $1 \mathrm{GeV}^{2}$ and was averaged over $E_{\gamma}$ from 2.5 to $5.8 \mathrm{GeV}$. The $\phi$ decay distributions in the helicity system (see Sec. III C) are shown in Fig. 25 for $E_{\gamma}>1.58 \mathrm{GeV}$ (threshold) and for two intervals of the production angle in the c.m. system.

\section{COMPARISON OF $\varrho, \omega$, AND $\phi$ PRODUCTION}

From the near constancy of the $\rho$ production cross section as a function of energy and from the $\rho$ decay angular distributions, it follows that one-pion exchange does not contribute noticeably to $\rho$ production above $2 \mathrm{GeV}$. This conclusion has already been discussed in a previous paper ${ }^{6}$ and is confirmed by the complete data presented here. On the other hand, the constancy

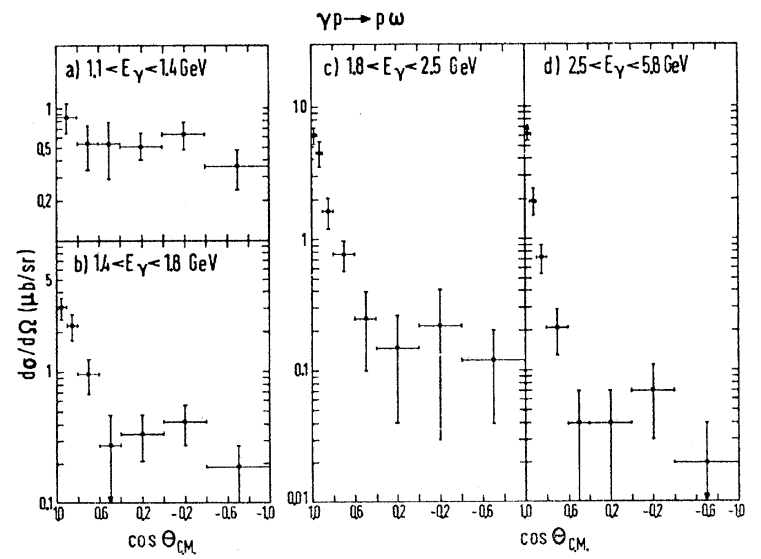

FIG. 19. Reaction $\gamma p \rightarrow p \omega$. Differential cross section $d \sigma / d \Omega$ for four photon-energy intervals; $\theta_{\text {c.m. }}$ is the angle between incoming and outgoing proton in the c.m. system. The arrow in Fig. 19(d) indicates possible scanning losses due to short proton tracks. of the cross section and the forward peaking of the production angular distribution is well in accordance with a diffractionlike mechanism. Figure 8 gives for the cross section for $\rho$ diffraction production above 3 $\mathrm{GeV}$

$$
\sigma_{\text {diff }}(\gamma p \rightarrow p \rho)=16.5 \pm 2.0 \mu \mathrm{b}
$$

In the case of $\omega$ production, neither a one-pion-exchange model nor a diffraction model alone seems to describe the data well. The fit to Eq. (10) mentioned in Sec. IV assumes that an incoherent mixture of both these processes is present. The estimate of $\sigma_{\text {diff }}$ from this method

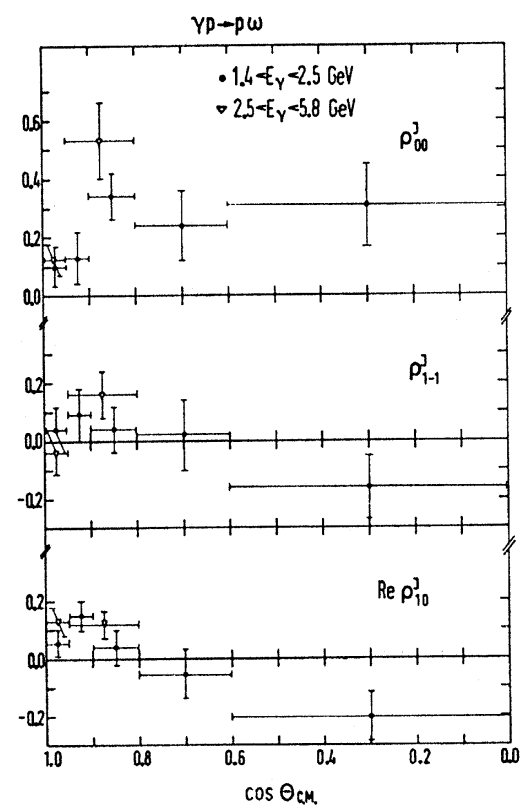

FIG. 20. Reaction $\gamma p \rightarrow p \omega$. Density matrix elements of the $\omega$ in the Jackson system (see text) as a function of the c.m. production angle $\cos \theta_{\text {c.m. }}$. The density matrix elements are given for two photon-energy intervals as indicated in the figure. 


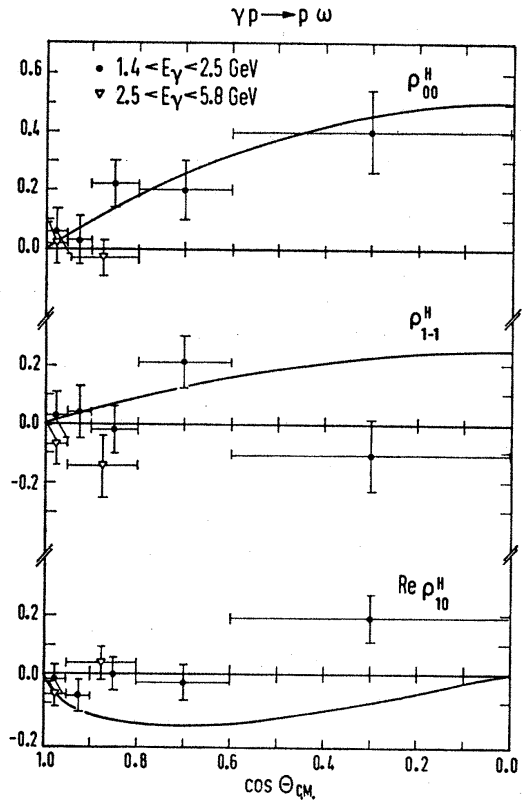

FIG. 21. Same as in Fig. 20 but in the helicity system. The curves are from Ref. 47. (See Sec. III C.)

is

$$
\sigma_{\text {diff }}(\gamma p \rightarrow p \omega)=1.7 \pm 0.8 \mu \mathrm{b}
$$

for $E_{\gamma}$ above $3 \mathrm{GeV}$. The error is statistical only, and does not take into account the fact that this picture is probably an oversimplification of the processes involved.

The errors of the $\phi$ production cross section [Fig. 23 (c) ] are very large due to the small number of events. The points are consistent with constancy. If we assume pure diffraction production, we find, above $E_{\gamma}=3.5 \mathrm{GeV}$,

$$
\sigma_{\mathrm{diff}}(\gamma p \rightarrow p \phi)=0.45 \pm 0.13 \mu \mathrm{b}
$$

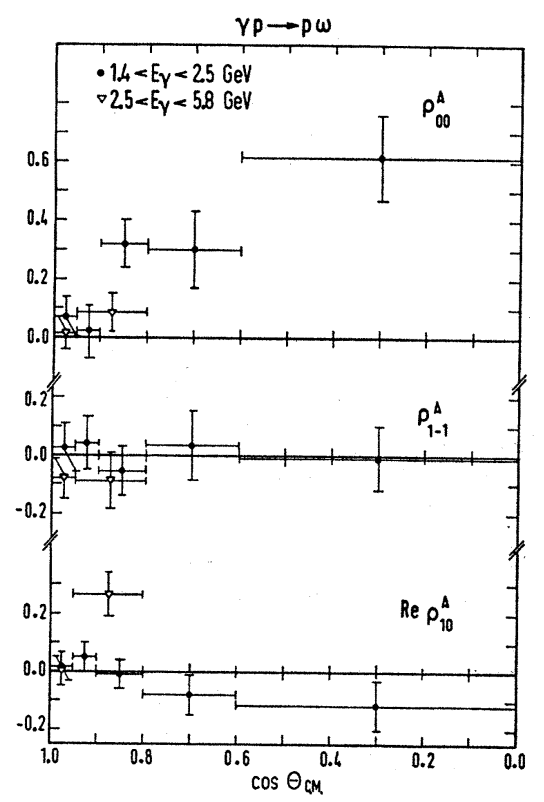

FIG. 22. Same as in Fig. 20 but in the Adair system.
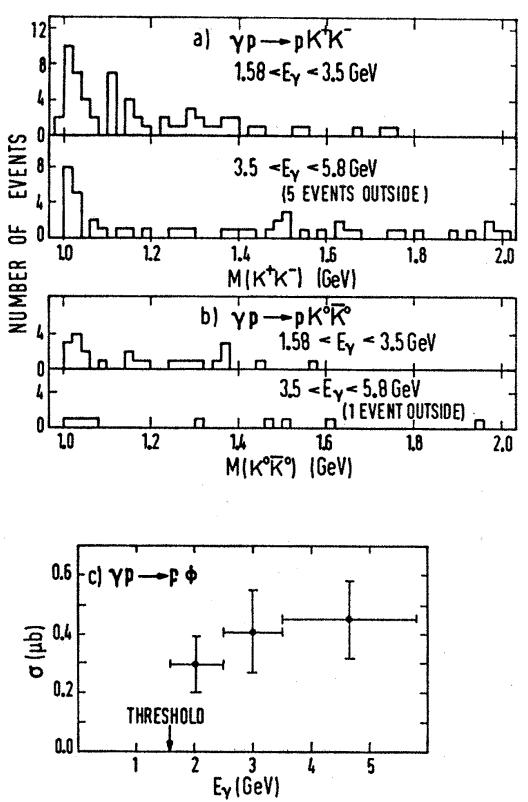

Frg. 23. Reaction $\gamma p \rightarrow p K^{+} K^{-}$and $\gamma p \rightarrow K^{0} \bar{K}^{0}$. Effectivemass distributions of (a) the $K^{+} K^{-}$combination and (b) the $K^{0} \bar{K}^{0}$ combination. (c) Total cross section for reaction $\gamma p \rightarrow p \phi$ as a function of the photon energy $E_{\gamma}$.

A way to describe the diffractive production of vector mesons is offered by the vector-dominance model (VDM), in which the incident photon is coupled to virtual vector mesons which are then scattered diffractively by the proton. (See Ref. 52 for a compilation of literature.) This model gives a relation between the cross section for diffractive photoproduction of vector mesons and vector-meson diffraction scattering by protons

$$
\sigma_{\text {diff }}(\gamma p \rightarrow p V) \simeq \frac{1}{4} \alpha\left(\gamma v^{2} / 4 \pi\right)^{-1} \sigma(V p \rightarrow V p),
$$

where $V$ signifies one of the vector mesons $\rho, \omega, \phi$, and $\alpha \simeq 1 / 137 . \gamma_{V}{ }^{-1}$ is the $V-\gamma$ coupling constant. Equation
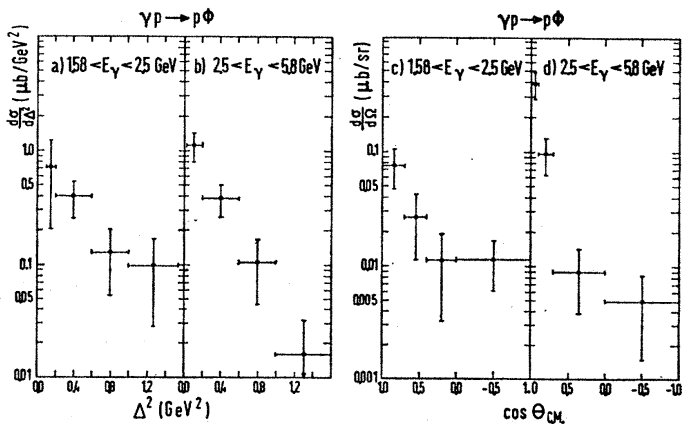

FIG. 24. Reaction $\gamma p \rightarrow p \phi$. (a) and (b) Differential cross sections $d \sigma / d \Delta^{2}$ for two photon-energy intervals. (c) and (d) Differential cross sections $d \sigma / d \Omega$ in the c.m. system for two photonenergy intervals.

${ }^{52}$ H. Joos, Acta Phys. Austriaca Suppl. IV, p. 320 (1967). 


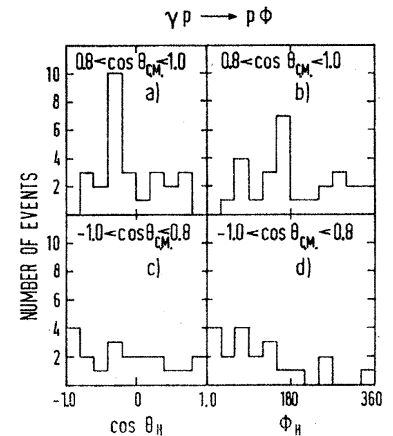

FIG. 25. Reaction $\gamma p \rightarrow p \phi$. Decay distributions in the helicity system for all events with $M(K \bar{K})<1.06 \mathrm{GeV}$ and all photon energies. No background is subtracted. (a) $\cos \theta_{H}$ distribution and (b) $\phi_{H}$ distribution for c.m. production angles $0.8<\cos \theta_{\mathrm{c} . \mathrm{m} .}<1.0$ (c) $\cos \theta_{H}$ distribution and (d) $\phi_{H}$ distribution for c.m. production angles $-1.0<\cos \theta_{\text {c.m. }}<0.8$. The angles $\theta_{H}$ and $\phi_{H}$ are defined in analogy to the ones for the $\rho^{0}$ meson. The distributions are not corrected for unobserved decays.

(11) holds also for differential cross sections, especially

$$
\begin{aligned}
& \left.\frac{d \sigma}{d \Delta^{2}}(\gamma p \rightarrow p V)\right|_{\Delta^{2}=0} \\
& \left.\simeq \frac{1}{4} \alpha\left(\frac{\gamma V^{2}}{4 \pi}\right)^{-1} \frac{d \sigma}{d \Delta^{2}}(V p \rightarrow V p)\right|_{\Delta^{2}=0} .
\end{aligned}
$$

In order to apply Eq. (12) for a comparison with our experimental results, one needs information on $\gamma_{V}$ and $d \sigma / d \Delta^{2}(V p \rightarrow V p)$. The coupling constant $\gamma_{\rho}$ has been determined directly from the leptonic $\rho$ decays ${ }^{53}$ and from a study of the reaction

$$
e^{+} e^{-} \rightarrow \pi^{+} \pi^{-}
$$

with storage rings. ${ }^{54,55}$ An average value is $\gamma_{\rho}{ }^{2} / 4 \pi$ $=0.47 \pm 0.12 . .^{56}$ The coupling constants for $\omega$ and $\phi$ have not yet been accurately measured. The most recent theoretical estimate gives the relation ${ }^{57}$

$$
\gamma_{\rho}^{-2}: \gamma_{\omega}^{-2}: \gamma_{\phi}^{-2}=9: 0.65: 1.33 \text {. }
$$

Values for the elastic vector-meson-proton diffraction cross sections in the forward direction can be obtained from the total vector-meson-proton cross sections using the optical theorem,

$$
\left.\frac{d \sigma}{d \Delta^{2}}(V p \rightarrow \mid r p)\right|_{\Delta^{2}=0}=\frac{\sigma_{T^{2}}(V p)}{16 \pi} .
$$

${ }^{53}$ S. C. C. Ting, in Proceedings of the 1967 International Symposium on Electron and Photon Interactions at High Energies (Stanford Linear Accelerator Center, Stanford, Calif., 1968), p. 452.

${ }_{54}$ V. L. Auslander, G. I. Budker, J. N. Pestov, V. A. Sidorov, A. N. Skrinsky, and A. G. Khabakhpashev, Phys. Letters 25B, 433 (1967).

${ }_{55} \mathrm{~J}$. E. Augustin, J. C. Bizot, J. Buon, J. Haissinski, D. Lalanne, P. C. Marin, J. Perez-y-Jorba, F. Rumpf, E. Silva, and S. Tavernier, Phys. Rev. Letters 20, 126 (1968).

${ }_{56}$ H. Joos, in Proceedings of the Heidelberg International Conference on Elementary Particles, edited by $\mathrm{H}$. Filthuth (NorthHolland Publishing Co., Amsterdam, 1968), p. 355.

${ }^{57}$ R. J. Oakes and J. J. Sakurai, Phys. Rev. Letters 19, 1266 (1967).
The total $\rho$-meson-nucleon and $\phi$-meson-nucleon cross sections have been measured recently by the absorption of these mesons in heavy nuclei. ${ }^{58}$ The results are in accordance with quark-model predictions. ${ }^{56,59-61} \mathrm{We}$ therefore use the quark-model cross sections $\sigma_{T}(\rho p)$ $=28 \mathrm{mb}, \sigma_{T}(\omega p)=28 \mathrm{mb}$, and $\sigma_{T}(\phi p)=11.5 \mathrm{mb}^{56}$ at about $4.5 \mathrm{GeV}$.

Inserting Eq. (14) into Eq. (12) and using the quarkmodel cross sections for $\sigma_{T}(V p)$ and Eq. (13) for $\left(\gamma_{v^{2}} / 4 \pi\right)$, with $\left(\gamma_{\rho}^{2} / 4 \pi\right)=0.47$, one obtains, for the right-hand side of Eq. (12),

$$
\begin{aligned}
d \sigma /\left.d \Delta^{2}\right|_{\Delta^{2}}=0 & =155 \mu \mathrm{b} / \mathrm{GeV}^{2} \text { for } \gamma p \rightarrow p \rho \\
& =11 \mu \mathrm{b} / \mathrm{GeV}^{2} \text { for } \gamma p \rightarrow p \omega \\
& =3.8 \mu \mathrm{b} / \mathrm{GeV}^{2} \text { for } \gamma p \rightarrow p \phi .
\end{aligned}
$$

The value for the $\rho$ meson is in good agreement with the extrapolated differential $\rho$-production cross section (see Table V) which has values between 130 and 150 $\mu \mathrm{b} / \mathrm{GeV}^{2}$. The value for the $\omega$ meson is also consistent with the photoproduction data given in Table VIII: At $4 \mathrm{GeV}$ the diffraction part of the $\omega$ cross section is estimated as roughly $50 \%$ of the total cross section, leading to a value of $\simeq 13 \mu \mathrm{b} / \mathrm{GeV}^{2}$, with a rather large error of $\pm 6 \mu \mathrm{b} / \mathrm{GeV}^{2}$.

The value for the $\phi$ meson of $3.8 \mu \mathrm{b} / \mathrm{GeV}^{2}$ should be compared with $1.6 \pm 0.6 \mu \mathrm{b} / \mathrm{GeV}^{2}$ from Table VIII.

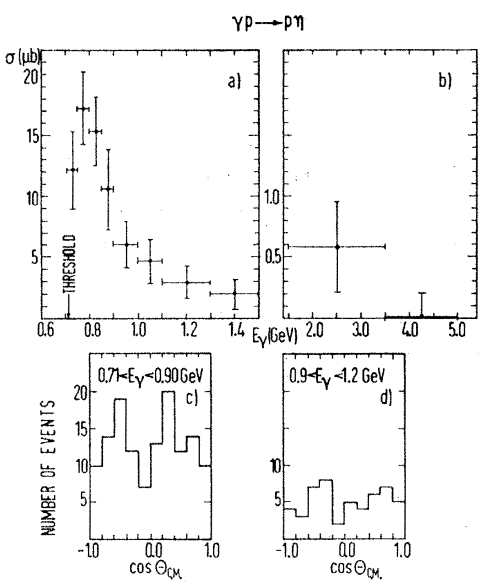

Frg. 26. Reaction $\gamma p \rightarrow p \eta$. (a) Total cross section as a function of the photon energy below $E_{\gamma}=1.5 \mathrm{GeV}$. (b) Total cross section above $E_{\gamma}=1.5 \mathrm{GeV}$. Production angular distribution in the center-of-mass system for two photon-energy intervals. (c) $0.71<E_{\gamma}<0.90 \mathrm{GeV}$ and (d) $0.9<E_{\gamma}<1.2 \mathrm{GeV}$. In Figs. 26(c) and 26 (d) all events in the mass region $0.52<M\left(\pi^{+} \pi^{-} \pi^{0}\right)<0.565 \mathrm{GeV}$ are included without background subtraction.

${ }^{58}$ J. G. Asbury, U. Becker, W. K. Bertram, P. Joos, M. Rohde, A. J. S. Smith, C. L. Jordan, and S. C. C. Ting, Phys. Rev. Letters 19, $865(1967) ; 20,1134(\mathrm{E})(1968)$; and S. C. C. Ting, DESY Report No. 68/29, 1968 (unpublished).

${ }^{59}$ H. Joos, Phys. Letters 24B, 103 (1967).

${ }^{60}$ K. Kajantie and J. S. Trefil, Phys. Letters 24B, 106 (1967).

${ }^{61}$ Theoretical attempts to derive vector-meson-nucleon cross sections have also been made with Regge-pole models [see P. G. O. Freund, Nuovo Cimento 44A, 411 (1966); 48A, 541 (1967)]; F. Buccella and M. Colocci, Phys. Letters 24B, 61 (1967). 
There is a discrepancy by roughly a factor of 2 . Since the input data suffer from large systematic $\left[\sigma_{T}(\phi p)\right]$ and statistical $[\sigma(\gamma p \rightarrow p \phi)]$ errors, it seems too early to say how serious this discrepancy is. ${ }^{61 a}$

The $\Delta^{2}$ dependence of $\rho, \omega$, and $\phi$ photoproduction has been discussed by Margolis. ${ }^{62}$ Margolis used the VDM and a broken-SU(3) quark model to relate differential cross sections $d \sigma / d \Delta^{2}(\gamma p \rightarrow p V)$ to differential cross sections for elastic $\pi^{+}, \pi^{-}$, and $K^{+}$scattering on protons. In this approach the experimental $\Delta^{2}$ dependence of $\gamma p \rightarrow p V$ is correctly reproduced up to $\Delta^{2}=1$ $\mathrm{GeV}^{2}$ for all three vector mesons.

The CEA group has fitted their data on $\rho$ and $\omega$ production with the strong-absorption model. ${ }^{19,46}$ Analogous fits to our differential cross sections for $\rho$ and $\omega$ production have been carried out by Moebes ${ }^{63}$ and give similar results for the parameters of the model [e.g., for $\rho$ production $R=(0.71 \pm 0.02) f$, $d=(0.09 \pm 0.01) f, \epsilon=0.3$ (constant), $\mu / \Delta=1.40 \pm 0.14$, $C_{\rho}=1.96 \pm 0.13$ with the notations of Ref. 46].

\section{PHOTOPRODUCTION OF OTHER MESONIC RESONANCES}

\section{A. Production of $\boldsymbol{\eta}$ and $X^{0}$}

Production of $\eta$ mesons is observed in the $\pi^{+} \pi^{-} \pi^{0}$ mass distributions of the reaction $\gamma p \rightarrow p \pi^{+} \pi^{-} \pi^{0}$ (see Fig. 16). Figure 26 shows the cross section for the reaction

$$
\gamma p \rightarrow p \eta
$$

as a function of $E_{\gamma}$. The experimental values are corrected for the unobserved decay modes of the $\eta$ using a branching ratio of $\Gamma\left(\eta \rightarrow \pi^{+} \pi^{-} \pi^{0}\right) / \Gamma(\eta \rightarrow$ all $)=0.224$. The cross section shows a characteristic peaking at about $0.8 \mathrm{GeV}$. This is generally attributed to the formation of an intermediate $S_{11}$ isobar state at 1570 $\mathrm{MeV}$ (see, e.g., Ref. 64). The c.m. production angular

61a Note added in proof. Recently, $\gamma_{\rho}, \gamma_{\omega}$, and $\gamma_{\phi}$ have been determined from leptonic decays and storage-ring experiments. The resulting a verage values are $\gamma_{\rho}^{2} / 4 \pi=0.52_{-0.06}^{+0.07}$ and $\gamma_{\rho}{ }^{-2}: \gamma_{\omega}{ }^{-2}: \gamma_{\phi}{ }^{-2}$ $=9:(1.00 \pm 0.21):\left(1.54 \ldots 0.40^{+0.43}\right)$. [S.C.C. Ting, rapporteur's talk at the Fourteenth International Conference on High-Energy Physics, Vienna 1968, DESY Internal Report No. F31/4, 1968 (unpublished)]. Taking these values of $\gamma_{V}$ instead of the above used ones, one calculates for the right-hand side of Eq. (12) $d \sigma / d \Delta^{2} \mid \Delta^{2}=0$ $=(140 \pm 14),(15.3 \pm 3.6)$, and $(4.0 \pm 1.1) \mu \mathrm{b} / \mathrm{GeV}^{2}$ for $\rho, \omega$, and $\phi$ photoproduction, respectively, where the quoted errors are solely due to the uncertainty of $\gamma_{V}$. For $\rho$ and $\omega$ photoproduction one finds again agreement with our experimental results. New counter measurements of the differential cross section for $\phi$ photoproduction on protons have been reported at $5.2,6,13$, and $16 \mathrm{GeV}$, e.g. $d \sigma /\left.d \Delta^{2}(\gamma p \rightarrow p \phi)\right|_{\Delta^{2}=0}=(2.75 \pm 0.4) \mu \mathrm{b} / \mathrm{GeV}^{2}$ at $E_{\gamma}=5.2 \mathrm{GeV}$ [S. C. C. Ting (unpublished)]. Our points at $2.5<E_{\gamma}<5.8 \mathrm{GeV}$ agree within errors with the counter results at higher energies. The new value for the $\phi$ forward cross section at $5.2 \mathrm{GeV}$ seems to be compatible with the above calculated one, if one considers the uncertainty of $\gamma_{\phi}$ and $\sigma_{T}(\phi p)$.

62 B. Margolis, CERN Report No. TH-901, 1968 (unpublished).

${ }^{63} \mathrm{~J}$. Moebes, thesis, Physikalisches Institut, Bonn, Report No. 3-03, 1968 (unpublished).

${ }^{64}$ R. K. Logan and F. Uchiyama-Campbell, Phys. Rev. 153 1634 (1967).
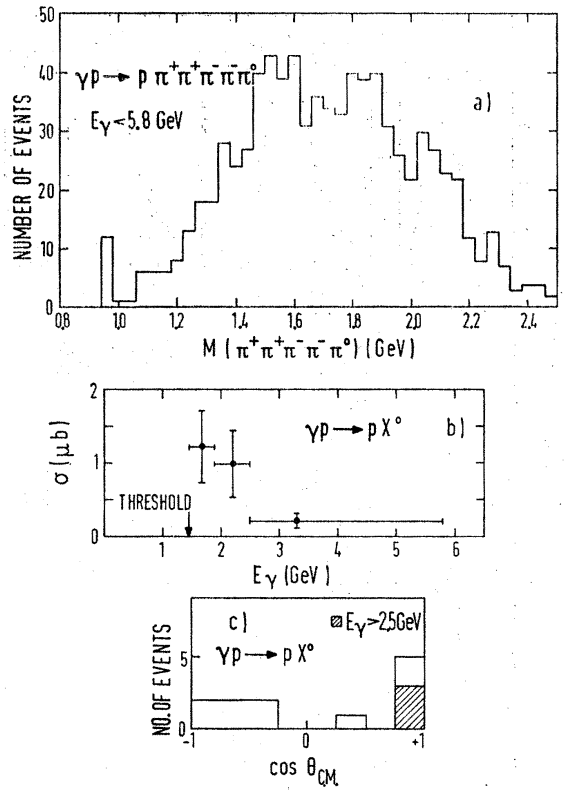

FIG. 27. Reaction $\gamma p \rightarrow p X^{0}$. (a) Effective-mass distribution of the $\pi^{+} \pi^{+} \pi^{-} \pi^{-} \pi^{0}$ combination from all events compatible with the hypothesis $\gamma p \rightarrow p \pi^{+} \pi^{+} \pi^{-} \pi^{-} \pi^{0}$. (b) Total cross section for $\gamma p \rightarrow p X^{0}$ as a function of the photon energy. (c) Production angular distribution in the c.m. system for the events in the $X^{0}$ mass region. The events with photon energies above $2.5 \mathrm{GeV}$ are shaded.

distribution of the $\eta$ is also shown in Fig. 26 for two $E_{\gamma}$ intervals.

The $\pi^{+} \pi^{+} \pi^{-} \pi^{-} \pi^{0}$ effective-mass distribution for the reaction

$$
\gamma p \rightarrow p \pi^{+} \pi^{+} \pi^{-} \pi^{-} \pi^{0}
$$

is shown in Fig. 27(a). There is evidence for the production of $X^{0}(958)$ whose decay mode

$$
\begin{aligned}
& X^{0} \rightarrow \eta \pi^{+} \pi^{-} \\
& \pi^{+} \pi^{-} \pi^{0}
\end{aligned}
$$

is observed. The cross section for $X^{0}$ production in the reaction

$$
\gamma p \rightarrow p X^{0}
$$

is shown in Fig. 27(b). The experimental values have been corrected for the unobserved decay modes of the $X^{0}$ using a branching ratio of ${ }^{41}$

$$
\Gamma\left(X^{0} \rightarrow \pi^{+} \pi^{+} \pi^{-} \pi^{-} \pi^{0}\right) / \Gamma\left(X^{0} \rightarrow \text { all }\right)=0.105 \text {. }
$$

The c.m. production angular distribution for the $X^{0}$ is shown in Fig. 27(c).

\section{B. Production of $f, f^{\prime}, A_{1}, A_{2}$, and $B$ Mesons}

We observe weak indications of $f(1260)$ meson production in the reaction $\gamma p \rightarrow p \pi^{+} \pi^{-}$, as can be seen from Figs. 7 and 40 for $2.5<E_{\gamma}<3.5 \mathrm{GeV}$. Estimates of the cross section for the reaction

$$
\gamma p \rightarrow p f
$$




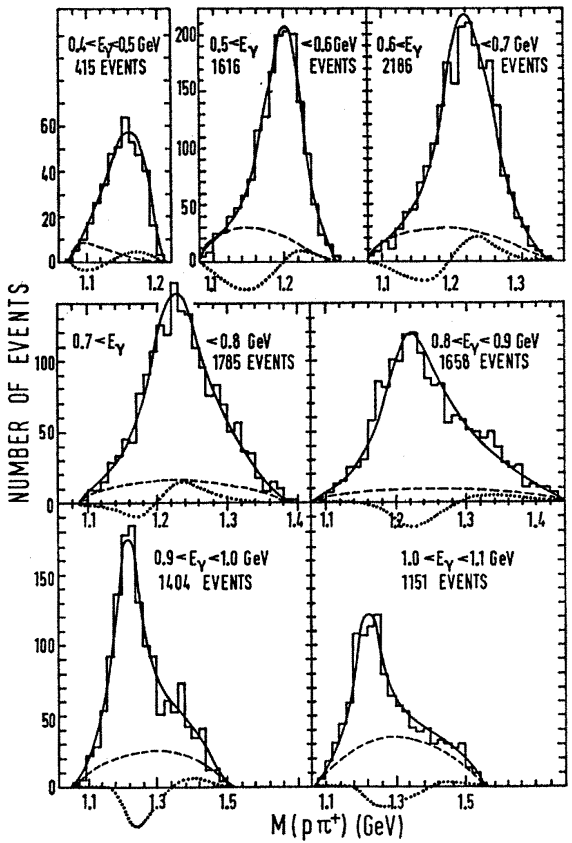

(a)

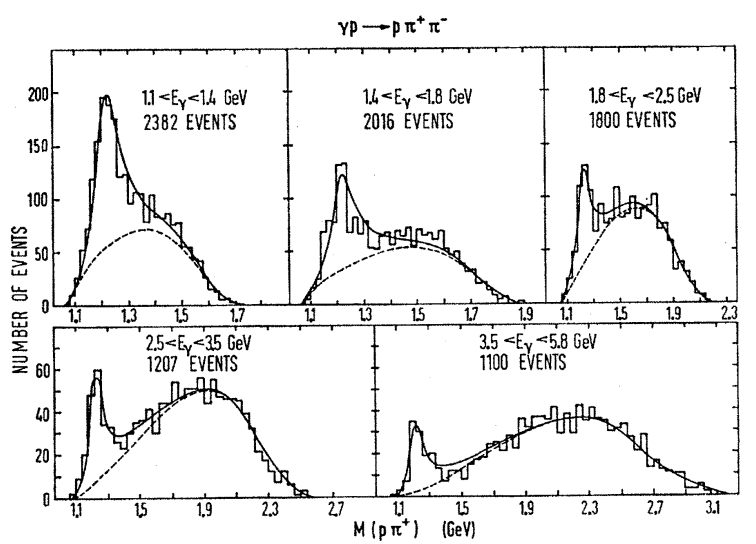

(b)

FIG. 28. Reaction $\gamma p \rightarrow p \pi^{+} \pi^{-}$. (a) Effective-mass distributions $M\left(p \pi^{+}\right)$for seven intervals of the photon energy $E_{\gamma}$ below 1.1 $\mathrm{GeV}$. The curves were obtained by the fitting procedure described in Sec. VIII A. The full curves are the sum of the contributions from Lorentz-invariant phase space, the resonance distribution for $\Delta^{++}$, and the reflection from $\gamma p \rightarrow \Delta^{0} \pi^{+}$including interference between $\Delta^{++}$and $\Delta^{0}$ amplitudes. For $1.0<E_{\gamma}<1.1 \mathrm{GeV}$ the full curve contains also the reflection from $\gamma p \rightarrow p \rho^{0}$. The dotted curves show the interference term. The dashed curves give the sum of phase space and the reflection from $\Delta^{0}$. For $1.0<E_{\gamma}<1.1$ $\mathrm{GeV}$ the dashed curve contains also the reflection from $\rho^{0}$. (b) Effective-mass distributions $M\left(p \pi^{+}\right)$for five intervals of the photon energy $E_{\gamma}$ above $1.1 \mathrm{GeV}$. The full curves are the sum of the contributions from Lorentz-invariant phase space, the resonance distribution for $\Delta^{++}$, and the reflections from $\gamma p \rightarrow \Delta^{0} \pi^{+}$ and $\gamma p \rightarrow p \rho^{0}$; the dashed curves show the sum of phase space and the reflections from $\Delta^{0}$ and $\rho^{0}$ production.

are listed in Table IX(a). The values have been corrected for the unobserved decay mode $f \rightarrow \pi^{0} \pi^{0}$ by a factor $\frac{3}{2}$. Figure 40 indicates that the events contributing to the $f^{0}$ signal are produced at large momentum transfers $\left(\Delta^{2}>0.3 \mathrm{GeV}^{2}\right)$.
We have looked for photoproduction of $f^{\prime}(1514)$ in the reaction

$$
\begin{aligned}
\gamma p \rightarrow p f^{\prime} & \\
& \searrow K^{+} K^{-} .
\end{aligned}
$$

Figure 23(a) shows the respective $K^{+} K^{-}$mass distribution. There are $3 \pm 2$ events above background at the position of the $f^{\prime}$ in the photon energy interval $3.5<E_{\gamma}<5.8 \mathrm{GeV}$. The resulting cross section is $0.15 \pm 0.09 \mu \mathrm{b}$. The value has been corrected for the non- $K^{+} K^{-}$decay modes by a factor $1 / 0.36 .{ }^{41}$

We have also looked for the photoproduction of $A_{1}$, $A_{2}$, and $B$ mesons in the reactions listed in Table IX(b). No significant production of these resonances was found. Table IX(b) gives upper limits for the cross sections of the reactions listed.

\section{THE REACTION $\gamma p \rightarrow \Delta(1236) \pi$}

\section{A. Fitting Procedure}

As mentioned in Sec. III, we observe strong $\Delta^{++}(1236)^{65}$ production in the reaction

$$
\gamma p \rightarrow p \pi^{+} \pi^{-} \text {. }
$$

Figures 28 and 29 show the $p \pi^{+}$and $p \pi^{-}$effectivemass distributions for twelve $E_{\gamma}$ intervals. There is evidence for the production of $\Delta^{++}$in the reaction

$$
\begin{array}{r}
\gamma p \rightarrow \Delta^{++} \pi^{-} \\
\searrow p \pi^{+}
\end{array}
$$

in all energy intervals, whereas the evidence for $\Delta^{0}$ production in

is weak.

$$
\begin{array}{r}
\gamma p \rightarrow \Delta^{0} \pi^{+} \\
\searrow \pi^{-}
\end{array}
$$

Below $1 \mathrm{GeV}$ (i.e., below the $\rho$ threshold) the $\Delta^{0}$ and $\Delta^{++}$bands in the Dalitz plot for reaction (1a) overlap strongly. Therefore it is important to take possible

\begin{tabular}{|c|c|c|c|}
\hline Reaction & $\begin{array}{c}E_{\gamma} \\
(\mathrm{GeV})\end{array}$ & $\begin{array}{c}\sigma \\
(\mu \mathrm{b})\end{array}$ & $\begin{array}{l}\text { Confidence } \\
\text { level }(\%)\end{array}$ \\
\hline & (a) & & \\
\hline$\gamma p \rightarrow p f$ & $\begin{array}{l}2.5-3.5 \\
3.5-4.5 \\
4.5-5.8\end{array}$ & $\begin{array}{l}0.90 \pm 0.45 \\
0.40 \pm 0.30 \\
0.06 \pm 0.30\end{array}$ & \\
\hline$\gamma p \rightarrow p f^{\prime}$ & $\begin{array}{r}3.5-5.8 \\
\text { (b) }\end{array}$ & $0.15 \pm 0.09$ & \\
\hline $\begin{array}{l}\gamma p \rightarrow p A_{1}{ }^{0} \\
\gamma p \rightarrow p A_{2}{ }^{0}\end{array}$ & $\begin{array}{l}1.7-5.8 \\
2.2-5.8\end{array}$ & $\begin{array}{l}<0.35 \\
<0.35\end{array}$ & $\begin{array}{l}90 \\
90\end{array}$ \\
\hline 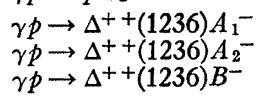 & $\begin{array}{l}2.5-5.8 \\
2.9-5.8 \\
2.7-5.8\end{array}$ & $\begin{array}{l}<0.35 \\
<0.40 \\
<0.15\end{array}$ & $\begin{array}{l}90 \\
90 \\
90\end{array}$ \\
\hline
\end{tabular}

TABlE IX. (a) Cross sections for reactions $\gamma p \rightarrow p f^{0}$ and $\gamma p \rightarrow p f^{\prime}$. (b) Upper limits for the cross sections of $A_{1}, A_{2}$, and $B$ meson production.

${ }^{65}$ In the following, we abbreviate $\Delta(1236)$ by $\Delta$. 
TABLE X. Reaction $\gamma p \rightarrow \Delta^{++} \pi^{-}$. Differential cross sections $d \sigma / d \Delta^{2}\left(\mu \mathrm{b} / \mathrm{GeV}^{2}\right)$ for various intervals of the photon energy.

\begin{tabular}{|c|c|c|c|c|c|c|c|}
\hline$\Delta^{2}\left(\mathrm{GeV}^{2}\right) \backslash E_{\gamma}(\mathrm{GeV})$ & $0.4-0.5$ & $0.5-0.6$ & $0.6-0.7$ & $0.7-0.8$ & $0.8-0.9$ & $0.9-1.0$ & $1.0-1.1$ \\
\hline $\begin{array}{c}\left(\Delta^{2}\right)_{\min }-0.05 \\
0.05-0.1 \\
0.1-0.15 \\
0.15-0.2 \\
0.2-0.25 \\
0.25-0.3 \\
0.3-0.35 \\
0.35-0.4 \\
0.4-0.45 \\
0.45-0.5\end{array}$ & $\begin{array}{l}60 \pm 10 \\
76 \pm 10 \\
43 \pm 7 \\
13 \pm 5\end{array}$ & $\begin{array}{r}193 \pm 18 \\
311 \pm 18 \\
200 \pm 15 \\
102 \pm 12 \\
53 \pm 9 \\
14 \pm 6 \\
9 \pm 7 \\
3 \pm 4\end{array}$ & $\begin{array}{r}225 \pm 21 \\
340 \pm 27 \\
270 \pm 23 \\
202 \pm 19 \\
146 \pm 15 \\
107 \pm 13 \\
71 \pm 11 \\
45 \pm 9 \\
16 \pm 7\end{array}$ & $\begin{array}{l}156 \pm 17 \\
249 \pm 23 \\
166 \pm 21 \\
134 \pm 19 \\
102 \pm 17 \\
86 \pm 16 \\
81 \pm 15 \\
86 \pm 14 \\
40 \pm 10 \\
36 \pm 9\end{array}$ & $\begin{array}{r}117 \pm 19 \\
195 \pm 22 \\
193 \pm 20 \\
131 \pm 19 \\
119 \pm 18 \\
105 \pm 18 \\
48 \pm 17 \\
71 \pm 17 \\
51 \pm 15 \\
50 \pm 14\end{array}$ & $\begin{array}{r}96 \pm 18 \\
155 \pm 18 \\
136 \pm 18 \\
124 \pm 17 \\
122 \pm 16 \\
85 \pm 16 \\
58 \pm 14 \\
39 \pm 15 \\
29 \pm 15 \\
29 \pm 15\end{array}$ & $\begin{array}{r}90 \pm 17 \\
103 \pm 16 \\
74 \pm 16 \\
116 \pm 17 \\
83 \pm 14 \\
78 \pm 16 \\
50 \pm 13 \\
51 \pm 14 \\
47 \pm 13 \\
30 \pm 12\end{array}$ \\
\hline$\Delta^{2}\left(\mathrm{GeV}^{2}\right) \backslash E_{\gamma}(\mathrm{GeV})$ & $1.1-1.4$ & $1.4-1.8$ & $1.8-2.5$ & $2.5-3.5$ & $3.5-5.8$ & & \\
\hline $\begin{array}{r}\left(\Delta^{2}\right)_{\min }-0.1 \\
0.1-0.2 \\
0.2-0.3 \\
0.3-0.4 \\
0.4-0.5\end{array}$ & $\begin{array}{l}82 \pm 8 \\
60 \pm 8 \\
40 \pm 7 \\
14 \pm 6 \\
14 \pm 6\end{array}$ & $\begin{array}{r}81 \pm 6 \\
39 \pm 6 \\
16 \pm 4 \\
6 \pm 4 \\
5 \pm 4\end{array}$ & $\begin{array}{r}42 \pm 4 \\
19 \pm 4 \\
7 \pm 3 \\
0 \pm 1 \\
0 \pm 1\end{array}$ & $\begin{array}{r}25 \pm 3 \\
8 \pm 3 \\
2 \pm 1 \\
3 \pm 3 \\
0 \pm 3\end{array}$ & $\begin{array}{r}10 \pm 3 \\
5.5 \pm 2 \\
1.5 \pm 2 \\
1.5 \pm 2 \\
0 \pm 1\end{array}$ & & \\
\hline
\end{tabular}

interference effects between the $\Delta^{++}$and $\Delta^{0}$ production amplitudes into account. ${ }^{66}$ We have introduced the interference between the $\Delta^{++}$and $\Delta^{0}$ amplitudes in the following way:

The density distribution in the Dalitz plot was described as a function of the photon energy $E_{\gamma}$ by the expression

$$
\begin{aligned}
& {\left[d N / d M^{2}\left(p \pi^{+}\right) d M^{2}\left(p \pi^{-}\right) d E_{\gamma}\right]} \\
& =\left[S\left(E_{\gamma}\right) / E_{\text {c.m. }}{ }^{2} E_{\gamma}\right]\left[A_{++}{ }^{2}\left|B_{++}\right|{ }^{2}+A_{0}{ }^{2}\left|B_{0}\right|^{2}\right. \\
& \quad+2 \alpha A_{++} A_{0} \operatorname{Re}\left(B_{++} B_{0}{ }^{*}\right)+A_{\left.\mathrm{Ps}^{2}{ }^{2} C_{\mathbf{P s}^{2}}\right] .}
\end{aligned}
$$

With the normalizations described below, $A_{++}$and $A_{0}$ are the moduli of the $\Delta^{++}$and $\Delta^{0}$ contributions to the total amplitude. The functions $B_{++}$and $B_{0}$ are Breit-Wigner amplitudes; for instance, for the $\Delta^{++37}$ :

$$
\begin{gathered}
B_{++}\left(M^{2}\left(p \pi^{+}\right), M^{2}\left(p \pi^{-}\right), E_{\gamma}\right)=C_{++} b_{++}, \\
b_{++}=\left(\frac{M\left(p \pi^{+}\right) \Gamma W}{q}\right)^{1 / 2} \frac{e^{i \phi}+}{\left[M_{\Delta^{2}}-M^{2}\left(p \pi^{+}\right)\right]+i M_{\Delta} \Gamma} .
\end{gathered}
$$

$C_{++}$is the normalization constant determined by integrating over the whole Dalitz plot and over the $E_{\gamma}$ interval used:

$$
C_{++}=(n / I)^{1 / 2},
$$

$n$ is the total number of events in the $E_{\gamma}$ interval,

$$
I=\int \frac{S\left(E_{\gamma}\right)}{E_{\mathrm{c} . \mathrm{m} .}{ }^{2} E_{\gamma}}\left|b_{++}\right|^{2} d M^{2}\left(p \pi^{-}\right) d M^{2}\left(p \pi^{+}\right) d E_{\gamma},
$$

$M\left(p \pi^{+}\right)$is the effective mass of $p \pi^{+}, M_{\Delta}$ is the mass of the $\Delta$ isobar $=1.236 \mathrm{GeV}, \Gamma$ is the energy-dependent width [formulas (A1) and (A6) of Ref. 37 with $\Gamma_{0}=0.120$ $\mathrm{GeV}$ and $a=2.2], q$ is the modulus of the three-momen-

${ }^{66}$ The introduction of interference into our fitting program could remove a difficulty which we encountered before. When we included $\Delta^{0}$ without interference, the fit gave negative values for the $\Delta^{0}$ contribution in most of the $E_{\gamma}$ intervals. tum of the proton in the $\left(p \pi^{+}\right)$c.m. system, and $W\left(M^{2}\left(p \pi^{+}\right), M^{2}\left(p \pi^{-}\right), E_{\gamma}\right)$ is the decay distribution of the $\Delta^{++}$in the helicity system. In this system one has a linear relation between the cosine of the decay angle and $M^{2}\left(p \pi^{-}\right)$. For an isotropic distribution $W=$ constant; $\phi_{++}$is the phase angle, $S\left(E_{\gamma}\right)$ is the energy spectrum of the photons, and $E_{\text {c.m. }}$. is the total energy in the over-all c.m. system. The factor $1 / E_{\gamma}$ in Eq. (15) is due to the flux factor.

The definition of the Breit-Wigner amplitude $B_{0}$ is completely analogous to $B_{++}$. The constant phase-space background $C_{\mathrm{Ps}^{2}}{ }^{2}$ is normalized corresponding to Eq. (16) and added incoherently to the Breit-Wigner terms with a weight $A_{\mathrm{Ps}^{2}}{ }^{2}$. The factor $\alpha$ which appears in the interference term of Eq. (15) was introduced for the following reason: In the Dalitz plot $M^{2}\left(p \pi^{+}\right)$versus $M^{2}\left(p \pi^{-}\right)$the effects of interference may partly have been cancelled, since only two of the five kinematical variables are considered, which characterize a threebody final state. Therefore $0 \leq \alpha \leq 1$ is expected. Equation (15) was fitted to the data using the maximumlikelihood method. The quantities determined by the fit are $A_{++}, A_{0}, A_{\mathrm{PS}}, \alpha$, and the phase difference $\phi=\phi_{++}-\phi_{0}$. In a first series of fits we found $\alpha$ to be well compatible with $\alpha=1$. For our final fits we therefore kept $\alpha=1$ fixed. Details of the fitting program will be given in Ref. 67.

We have checked the stability of the fits by repeating the calculations with several small modifications on the function $N\left(M^{2}\left(p \pi^{+}\right), M^{2}\left(p \pi^{-}\right), E_{\gamma}\right)$ to be fitted: For the decay distribution $W$, various assumptions were tried: (i) isotropic decay, (ii) decay according to the prediction of the Stichel-Scholz model, ${ }^{68}$ (iii) decay according to a best fit to the experimental decay dis-

${ }^{67}$ W. P. Swanson, D. Lüke, and H. Spitzer, DESY Report, 1968 (in preparation)

${ }^{68}$ P. Stichel and M. Scholz, Nuovo Cimento 34, 1381 (1964). (Formulas without high-energy and small-angle approximations are given in Ref. 70.) 


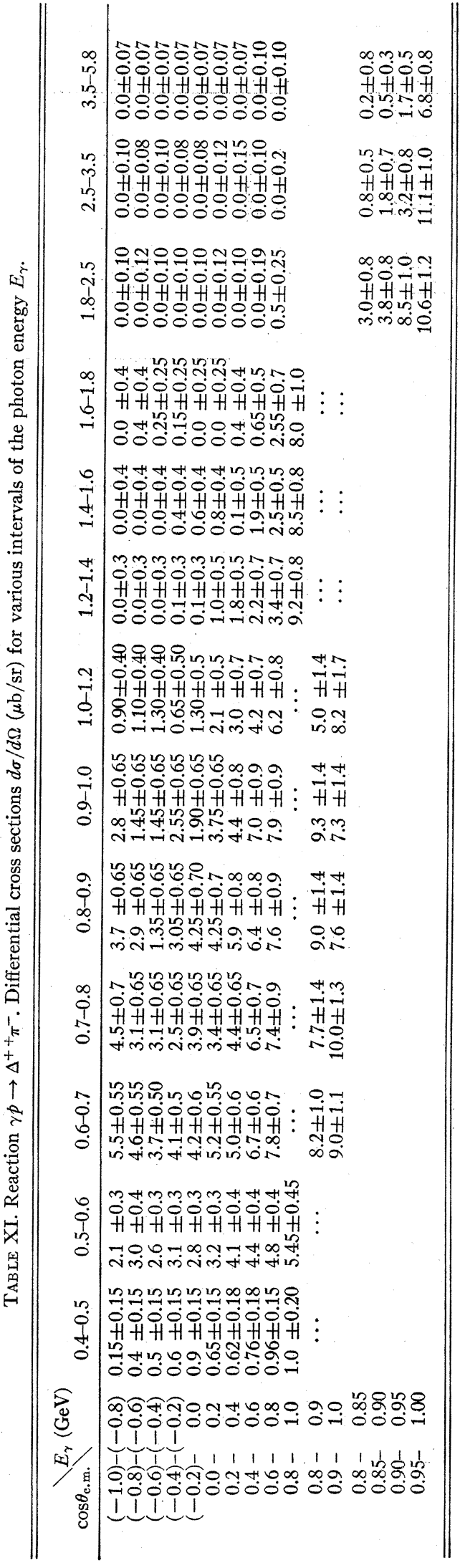

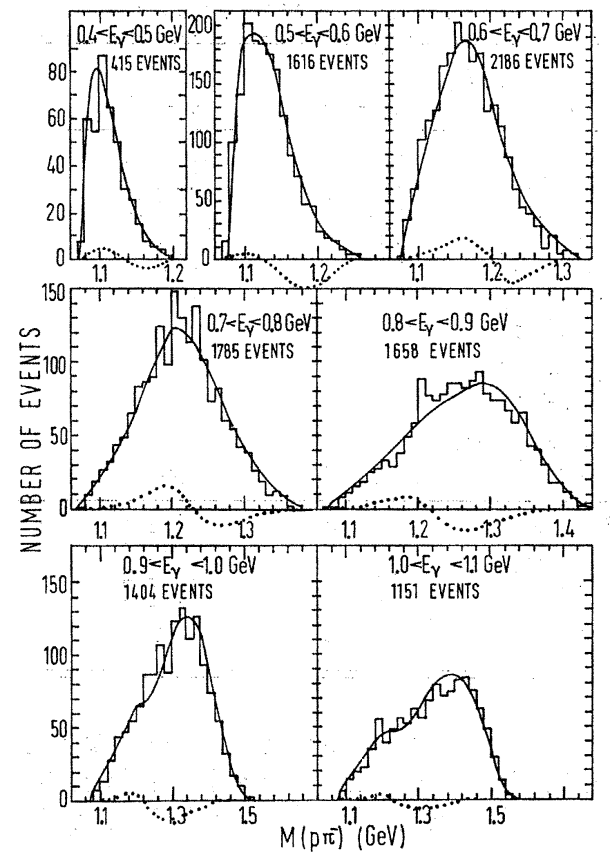

(a)

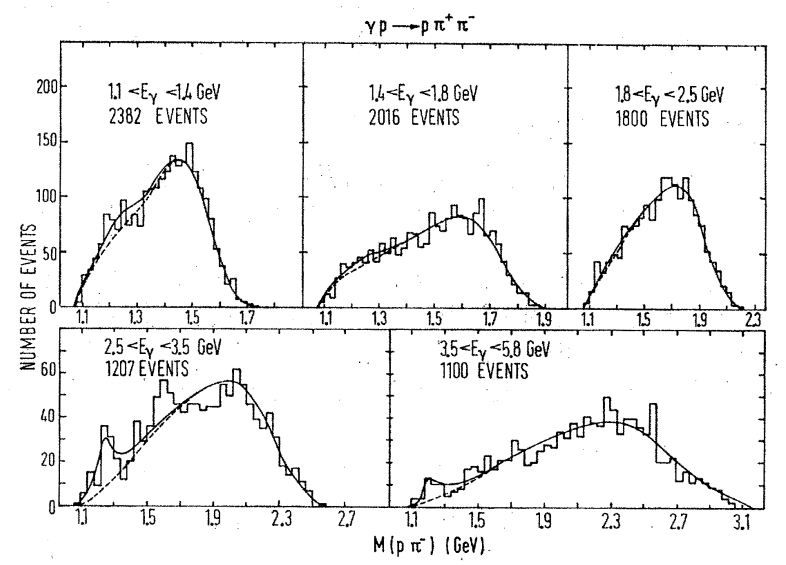

(b)

FIg. 29. Reaction $\gamma p \rightarrow p \pi^{+} \pi^{-}$. (a) Effective-mass distributions $M\left(p \pi^{-}\right)$for seven intervals of the photon energy $E_{\gamma}$ below 1.1 $\mathrm{GeV}$. The curves were obtained by the fitting procedure described in Sec. VIII A. The full curves show the sum of Lorentz-invariant phase space, the resonance distribution for $\Delta^{0}$, and the reflection from $\gamma p \rightarrow \Delta^{++} \pi^{-}$including interference between $\Delta^{++}$and $\Delta^{0} \mathrm{am}-$ plitudes. For $1.0<E_{\gamma}<1.1 \mathrm{GeV}$ the full curve contains also the reflection from $\gamma p \rightarrow p \rho^{0}$. The dotted curves show the interference term. (b) Analogous as in Fig. 28(b) but for $M\left(p \pi^{-}\right)$.

tributions. For the background distribution $C_{\mathrm{PS}}$, we have tried (i) Lorentz-invariant phase space and (ii) Lorentz-invariant phase space modified by an assumed $S$-wave $\pi \pi$ interaction. Also, in some fits the phase difference $\phi$ was kept fixed at $180^{\circ}$. All these modifications produced no changes outside the statistical errors in the values of the fitted parameters.

This fitting method was used below a photon energy of $1.1 \mathrm{GeV}$ to find the total cross sections for reactions (1c) and (1d) as well as the interference term. The dif- 


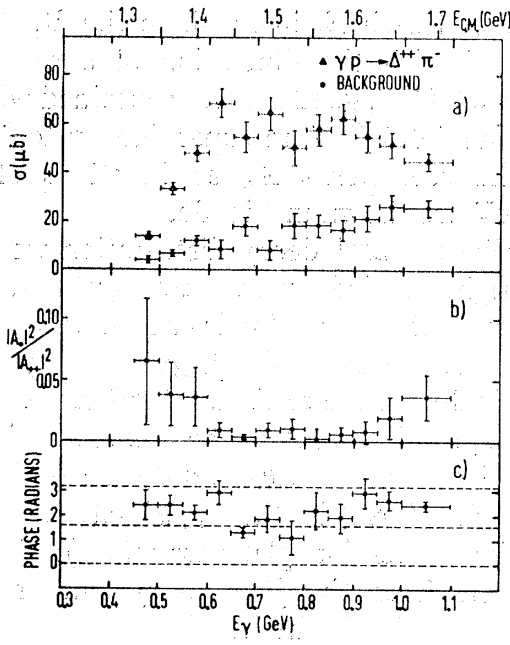

FIG. 30. Reaction $\ddot{\gamma} p \rightarrow p \pi^{+} \pi^{-}$. Cross section for the production of $\Delta(1236)$ as a function of the photon energy $E_{\gamma}$ below $1.1 \mathrm{GeV}$. (a) $\boldsymbol{\Delta}$ : cross section for $\gamma p \rightarrow \Delta^{++} \pi^{-}$; $\phi$ : cross section for phasespace background. (b) $A_{0}$ : amplitude for $\gamma p \rightarrow \Delta^{0} \pi^{+} ; A_{++}$: amplitude for $\gamma p \rightarrow \Delta^{++} \pi^{-} ; \quad \sigma\left(\gamma p \rightarrow \Delta^{0} \pi^{+}\right)-\left(\left|A_{0}\right|^{2} /\left|A_{++}\right|^{2}\right)$ $\times \sigma\left(\gamma p \rightarrow \Delta^{++} \pi^{-}\right)$. (c) Phase angle $\phi$ between the $\Delta^{0}$ and the $\Delta^{++}$ amplitudes as a function of the photon energy $E_{\gamma,} \phi=\phi_{++}-\phi_{0}$.

ferential production cross sections and the decay angular distributions for the $\Delta^{++}[$reaction (1c)] as well as the total cross section above $1.1 \mathrm{GeV}$ were obtained without taking interference into account. [We used the same fitting programs as for reaction $\gamma p \rightarrow p \rho$ (see Appendix).]

\section{B. Results}

Figure 30 shows the cross section for reaction (1c), the background contribution, the ratio $R=\left|A_{0}\right|^{2} \mid$ $\left|A_{++}\right|^{2}$ of the cross sections for $\Delta^{0}$ (decaying into $\left.p \pi^{-}\right)$and $\Delta^{++}$production [see Eq. (15)], and the phase difference $\phi$ as functions of $E_{\gamma}$ below $1.1 \mathrm{GeV}$. The crosssection ratio $R$ is much smaller than the value $R=\frac{1}{9}$ (a factor $\frac{1}{3}$ comes from the decay branching ratio of $\left.\Delta^{0} \rightarrow p \pi^{-}\right)$predicted by the OPE model, quark models, or a model with an intermediate $I=\frac{1}{2}$ isobar.

Figure 31 shows the cross section for $\Delta^{++}$production [reaction (1c)] for photon energies up to $5.8 \mathrm{GeV}$.
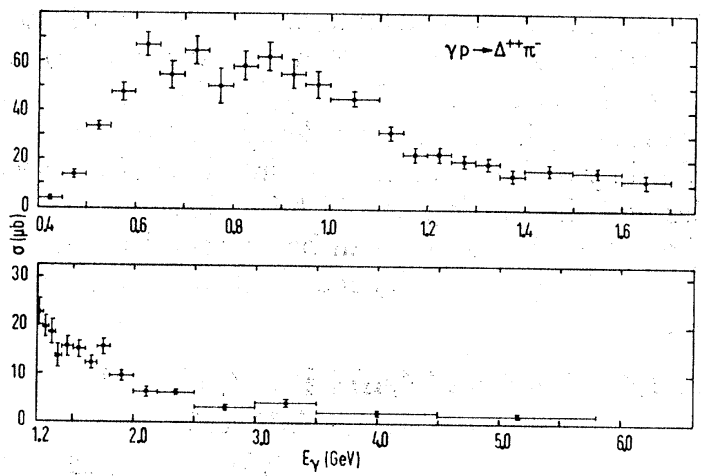

FIG. 31. Cross section for $\gamma p \rightarrow \Delta^{++} \pi^{-}$as a function of the photon energy up to $5.8 \mathrm{GeV}$.
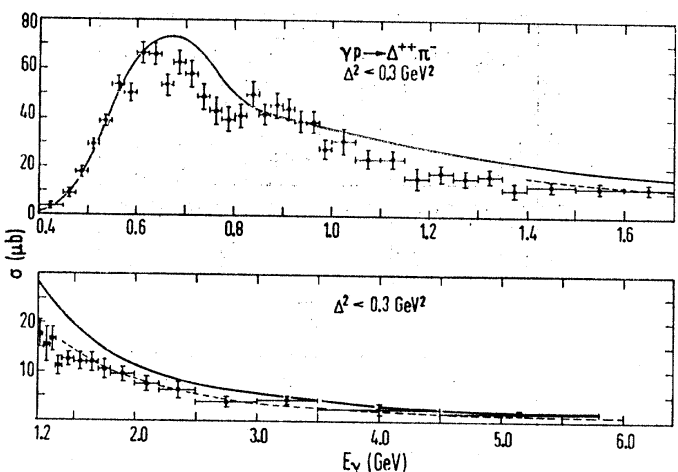

FIg. 32. Reaction $\gamma p \rightarrow \Delta^{++} \pi^{-}$. Cross section for $\Delta^{2}<0.3 \mathrm{GeV}^{2}$, where $\Delta^{2}$ is the square of the four-momentum transfer between incoming proton and $\Delta^{++}$. The full curves are predictions from a model by Lüke et al. (Ref. 70). The dashed curve is an OPE model plus gauge-invariance additions plus absorptive corrections (Ref. 71) (see text).

Figure 32 shows the same cross section for $\Delta^{2}\left(p / \Delta^{++}\right)$ $<0.3 \mathrm{GeV}^{2}$. Figure 33 shows the cross section for the production of $\Delta^{0} \pi^{+}$[reaction (1d)] at photon energies $E_{\gamma}>1.1 \mathrm{GeV}$. It was obtained by neglecting interferences between the amplitudes for $\Delta^{++}, \Delta^{0}$, and $\rho^{0}$ production. Similar $\Delta^{0} \pi^{+}$cross sections were obtained when an interference between $\Delta^{0}$ and $\rho^{0}$ amplitudes was assumed. Comparing the cross sections for reactions (1c) and (1d), one finds a ratio

$$
\underset{\searrow}{\left[\sigma\left(\gamma p \rightarrow \Delta^{0} \pi^{+}\right) / \sigma\left(\gamma p \rightarrow \Delta^{++} \pi^{-}\right)\right]}
$$

compatible with $\frac{1}{9}$ above $1.1 \mathrm{GeV}$ photon energy.
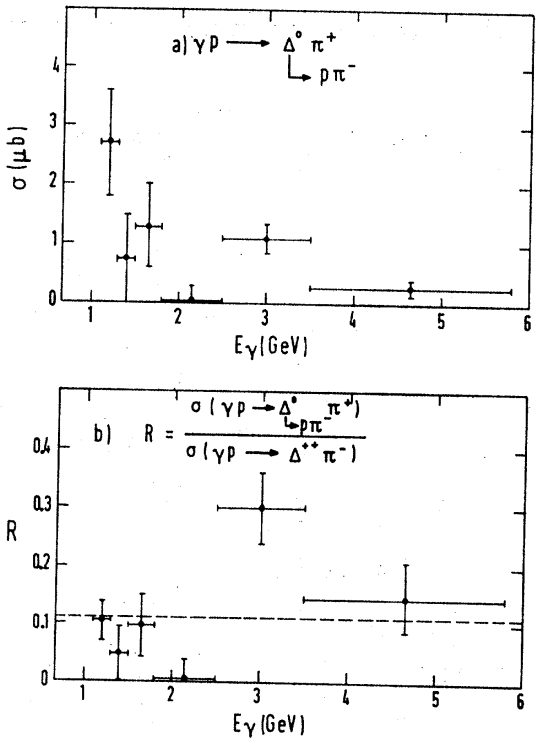

FIG. 33. (a) Cross section for $\gamma p \rightarrow \Delta^{0} \pi^{+}$(with $\Delta^{0} \rightarrow p \pi^{-}$) as a function of the photon energy above $1.1 \mathrm{GeV}$. (b) Ratio $\sigma\left(\gamma p \rightarrow \Delta^{0} \pi^{+}\right) / \sigma\left(\gamma p \rightarrow \Delta^{++} \pi^{-}\right)$as a function of the photon energy $p \pi^{-}$

above $1.1 \mathrm{GeV}$. The dashed line shows the ratio $\frac{1}{9}$. 


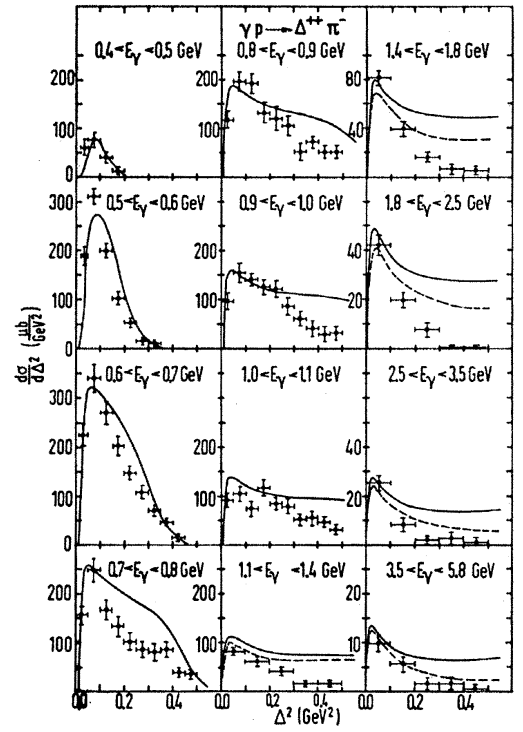

FIG. 34. Reaction $\gamma p \rightarrow \Delta^{++} \pi^{-}$. Differential cross sections $d \sigma / d \Delta^{2}$ for various intervals of the photon energy $E_{\gamma}$. The curves have the same meaning as in Fig. 32 .

The differential cross sections $d \sigma / d \Delta^{2}$ and $d \sigma / d \Omega$ (in the c.m. system) for $\Delta^{++}$production are shown in Figs. 34 and 35 for various $E_{\gamma}$ intervals. The corresponding numbers are given in Tables $\mathrm{X}$ and $\mathrm{XI}$. The differential cross section $d \sigma / d \Omega$ was fitted to an expansion in Legendre polynomials:

$$
d \sigma / d \Omega=(1 / k)^{2} \sum_{n=0}^{N} A_{n} P_{n}\left(\cos \theta_{\text {c.m. }}\right),
$$

where $k$ is the incident c.m. momentum, taken at the

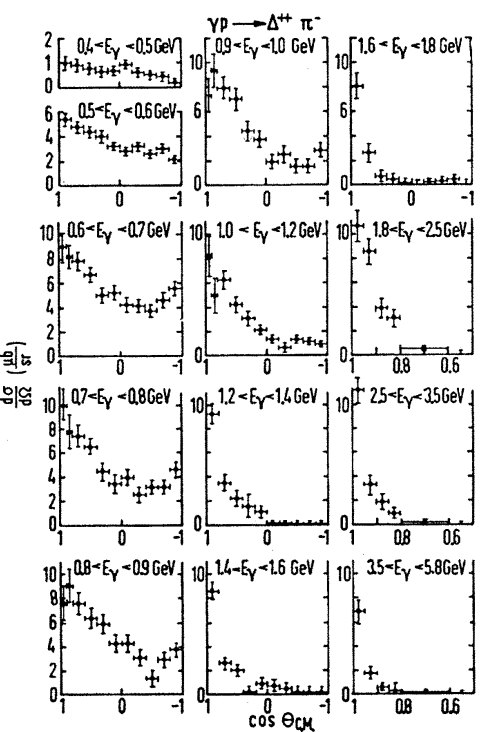

FIg. 35. Reaction $\gamma p \rightarrow \Delta^{++} \pi^{-}$. Differential cross sections $d \sigma / d \Omega$ for various intervals of the photon energy $E_{\gamma} . \theta_{\text {c.m. }}$ is the angle between incoming proton and $\Delta^{++}$in the c.m. system. central value of each $E_{\gamma}$ interval. In the fit, $(1 / k)^{2}$ has been inserted with the dimension of a cross section $(\mu \mathrm{b})$. Below $0.8 \mathrm{GeV}$, good fits were obtained with $N=2$, whereas around $1.8 \mathrm{GeV}$ an expansion up to $N=5$ was needed. The coefficients $A_{n}$ as functions of $E_{\gamma}$ for $E_{\gamma}<1.8 \mathrm{GeV}$ are shown in Fig. 36.

To describe the $\Delta^{++}$decay distribution we used two different right-handed coordinate systems. In the Jackson system the $z$ direction is chosen as the direction of the incident proton in the $p_{\text {out }} \pi^{+}$c.m. system. The $y$ direction is the normal to the production plane, defined by the cross product $\gamma \times \pi^{-}$of the momenta of $\gamma$ and $\pi^{-}$ in the $p_{\text {out }} \pi^{+}$c.m. system. $\theta_{J}$ and $\phi_{J}$ are the polar and azimuth angle of $p_{\text {out }}$ in the $p_{\text {out }} \pi^{+}$c.m. system with $\phi_{J}=0$ in the production plane ( $x z$ plane). The helicity system differs from the Jackson system only in the choice of the $z$ axis. In the helicity system the $z$ direction is chosen opposite to the direction of the $\pi^{-}$in the

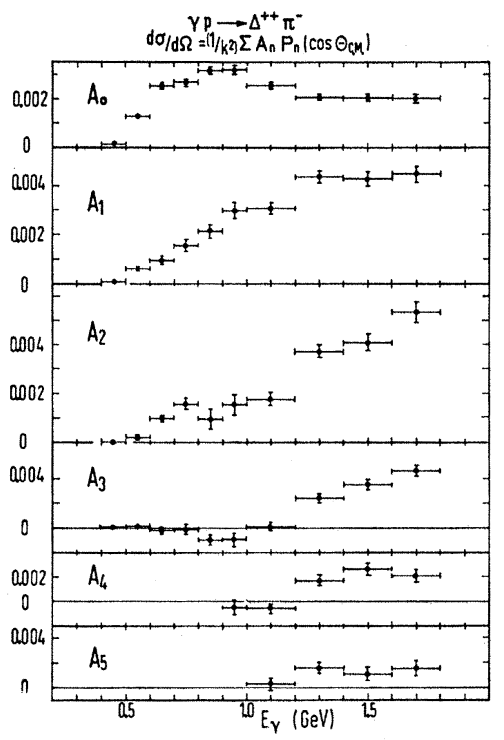

FIg. 36. Reaction $\gamma p \rightarrow \Delta^{++} \pi^{-}$. Coefficients $A_{n}$ of an expansion in Legendre polynomials fitted to the differential cross sections $d \sigma / d \Omega$, as functions of the photon energy $E_{\gamma}$ (see text).

$p_{\text {out }} \pi^{+}$c.m. system (i.e., equal to the direction of flight of the $\Delta^{++}$in the over-all c.m. system). Figure 37 shows the distributions of the $\Delta^{++}$decay angles $\cos \theta_{J}$ and $\phi_{J}$ in the Jackson system for $\Delta^{2}\left(p / \Delta^{++}\right)<0.3 \mathrm{GeV}^{2}$ and for various $E_{\gamma}$ intervals. The decay distributions were obtained by dividing all the data into bins of the decay angles and then fitting the $\Delta^{++}$fraction.

The decay distribution can be expressed in terms of the $\Delta^{++}$spin-space density matrix elements $\rho_{33}, \operatorname{Re} \rho_{3-1}$, $\operatorname{Re} \rho_{31}{ }^{44}$

$$
\begin{aligned}
W(\cos \theta, \phi)= & 3 / 4 \pi\left[\frac{1}{6}\left(1+4 \rho_{33}\right)+\frac{1}{2}\left(1-4 \rho_{33}\right) \cos ^{2} \theta\right. \\
& -(2 / \sqrt{3}) \operatorname{Re} \rho_{3-1} \sin ^{2} \theta \cos 2 \phi \\
& \left.-(2 / \sqrt{3}) \operatorname{Re} \rho_{31} \sin 2 \theta \cos \phi\right] .
\end{aligned}
$$

The three density matrix elements were determined by 
fitting this expression to the experimental data; the results are shown in Fig. 38 in the Jackson system and in Fig. 39 in the helicity system as functions of $E_{\gamma}$ for $\Delta^{2}\left(p / \Delta^{++}\right)<0.3 \mathrm{GeV}^{2}$. The density matrix elements vary rapidly between $E_{\gamma}=0.7$ and $1.5 \mathrm{GeV}$.

\section{Comparison with Theory}

Experimental results on the reaction $\gamma p \rightarrow \Delta^{++} \pi^{-}$can be compared with a number of models:

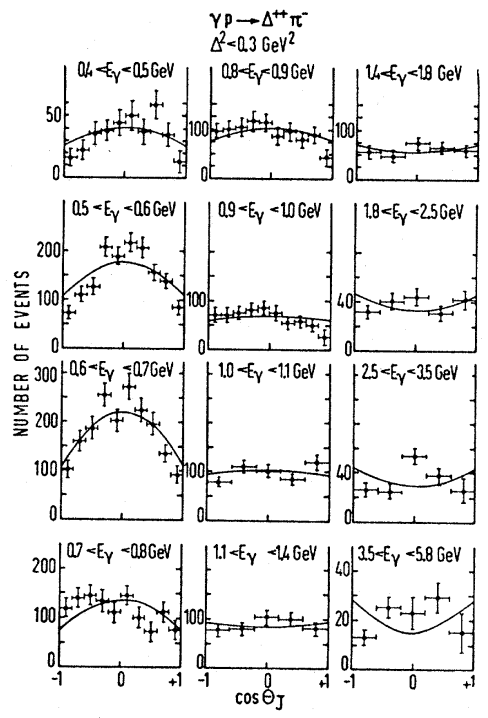

(a)

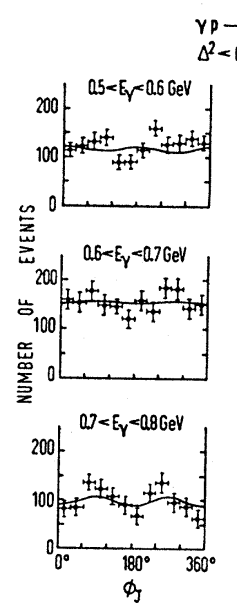

$Y p \rightarrow \Delta^{++} \pi$

$\Delta^{2}<0.36 \mathrm{~V}^{2}$
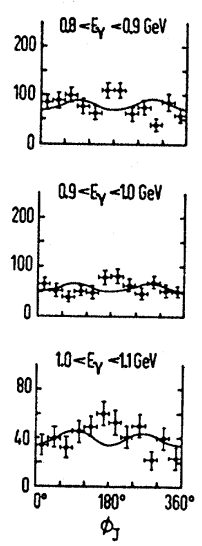

(b)

FIG. 37. Reaction $\gamma p \rightarrow \Delta^{++} \pi^{-}$for $\Delta^{2}<0.3 \mathrm{GeV}^{2}$. Decay angular distribution of the $\Delta^{++}$for various intervals of the photon energy $E_{\gamma}$. The curves have the same meaning as in Fig. 32. (a) Distributions of $\cos \theta_{J}$, in the Jackson system. (b) Distributions of $\phi_{J}$, the azimuth angle in the same system.

(1) Intermediate isobar model: This model assumes that the reaction proceeds via the formation of intermediate higher isobar states, which decay into $\Delta^{++} \pi^{-}$. A model containing the intermediate states $P_{11}(1420)$,

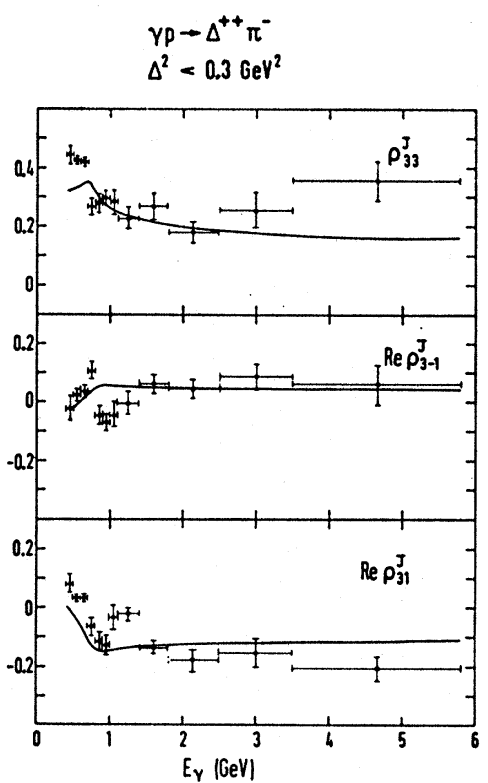

FIg. 38. Reaction $\gamma p \rightarrow \Delta^{++} \pi^{-}$for $\Delta^{2}<0.3 \mathrm{GeV}^{2}$. Decay density matrix elements in the Jackson system as functions of the photon energy $E_{\gamma}$. The curves have the same meaning as in Fig. 32.

$D_{13}$ (1512), $F_{15}$ (1688), and $F_{37}(1924)$ was used by the Cambridge Bubble Chamber Group ${ }^{22}$ to explain their experimental data. They found a rough agreement of the model with their data, however, with significant discrepancies in many details. Our data, when compared with their calculations, show the same discrepancies. ${ }^{69}$

(2) OPE model, with certain terms added by Stichel and $\mathrm{Scholz}^{68}$ to make the model gauge-invariant: Our preliminary data were compared with this model in a

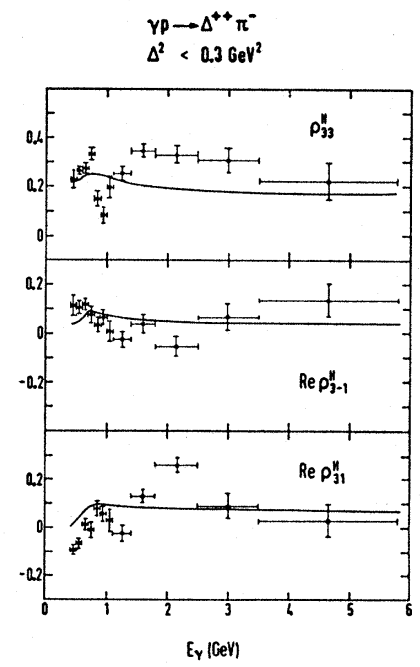

FIG. 39. Reaction $\gamma p \rightarrow \Delta^{++} \pi^{-}$for $\Delta^{2}<0.3 \mathrm{GeV}^{2}$. Decay density matrix elements in the helicity system as functions of the photon energy $E_{\gamma}$. The curves have the same meaning as in Fig. 32.

${ }^{69}$ A detailed comparison will be given in A. Meyer, Berlin (to be published). 
previous paper. ${ }^{4}$ Again, as for model (1), we found qualitative agreement with the data, but disagreement for a number of details.

(3) OPE model with the inclusion of higher isobar states [combination of models (1) and (2)]: As a first step, Scheunert and Stichel ${ }^{70}$ included the isobar states $P_{11}(1400)$ and $D_{13}(1525)$ in model (2). The strength of the contributions of these two isobars were determined by adjusting the model to the experimental $\Delta^{++}$-production cross section for $\Delta^{2}<0.3 \mathrm{GeV}^{2}$. The result of this fit is shown by the full curve in Fig. 32, which describes the data better than the OPE model alone. Also for the differential cross section the model yields a somewhat better agreement with experiment, at least for small values of the momentum transfer squared $\Delta^{2}$. This can be seen from the full curves in Fig. 34. The dashed curves in Figs. 32 and 34 are the results of the OPE model (2) with the inclusion of absorptive corrections. ${ }^{71}$ The predictions of model (3) for the decay angular distributions and the density matrix elements of the $\Delta^{++}$are shown by the curves in Figs. 37, 38, and 39.

(4) Independent-particle quark model. ${ }^{72}$ It has been used to predict the $\Delta^{++}$decay angular distribution at high energies and for forward production of the $\pi^{-}$. The prediction is

$$
W(\cos \theta) \propto 5-3 \cos ^{2} \theta .
$$

Our $\Delta^{++}$decay angular distributions in the Jackson system at high energies seem to be in agreement with this prediction, rather than with the prediction of the OPE model. However, it should be noted that our decay angular distributions in Fig. 37 are taken for $\Delta^{2}<0.3$ $\mathrm{GeV}^{2}$, i.e., not for the extreme forward direction.

(5) The threshold behavior of the cross section $\sigma\left(\gamma p \rightarrow \Delta^{++} \pi^{-}\right)$has been calculated by various authors starting from current algebra. All these calculations require additional assumptions if one wants numerical predictions which can be compared with experiment. Carruthers and Huang ${ }^{73}$ and Ebata ${ }^{74}$ have found that the main contribution to the amplitude of this process is given by the result of the static model of Cutkosky and Zachariasen. ${ }^{75}$ Our experimental cross sections near threshold $\left(E_{\gamma}<0.6 \mathrm{GeV}\right)$ exceed the prediction of Cutkosky and Zachariasen by a factor of $2-3$. By taking only the $s$-wave part of the experimental cross section the disagreement can be reduced.

Another approach with the help of current algebra was made by Narayanaswamy and Renner. ${ }^{76}$ They

${ }^{70}$ D. Lüke, M. Scheunert, and P. Stichel, DESY Report No. 68/7, 1968 (unpublished).

${ }_{71}$ M. P. Locher and W. Sandhas, Z. Physik 195, 461 (1966). ${ }^{72}$ K. Kajantie and J. S. Trefil, Nucl. Phys. B1, 648 (1967). ${ }^{73}$ P. Carruthers and H. W. Huang, Phys. Letters 24B, 464 (1967).

${ }^{74}$ T. Ebata, Phys. Rev. 154, 1341 (1967).

${ }_{75}$ R. E. Cutkosky and F. Zachariasen, Phys. Rev. 103, 1108 (1956).

${ }^{76} \mathbf{P}$. Narayanaswamy and B. Renner, Nuovo Cimento 53A. 107 (1968). treated the $\Delta^{++}$as a stable particle. This makes an exact numerical comparison of their prediction with our data difficult due to the finite width of the $\Delta^{++}$. If we take the effect of the finite width approximately into account, a rough comparison can be made. It turns out that our experimental cross section is very much larger (10-20 times) than the theoretical prediction.

\section{ACKNOWLEDGMENTS}

This work was made possible by the help of many individuals, whom we cannot all thank by name. Our special thanks go to the Synchrotron group and the Hallendienst at DESY and to the bubble-chamber crew for their excellent performance. We also appreciate greatly the help of Dr. R. Florent, Dr. G. Harigel, Dr. G. Linser, and Dr. R. I. Louttit, who were involved in starting this experiment. We thank Professor H. Joos, Professor G. Kramer, Professor P. Stichel, and Dr. K. Schilling for valuable discussions, and the computing centers in our individual laboratories for their services. We are also indebted to our scanners and technicians. The work in Aachen, Bonn, Hamburg, Heidelberg, and München was supported by the Bundesministerium für wissenschaftliche Forschung. One of us (W.P.S.) thanks the Volkswagen foundation for a fellowship.

\section{APPENDIX}

\section{A. Fitting Procedure for Reaction $\gamma p \rightarrow p \varrho^{0}$}

\section{Determination of Resonance Production}

The contributions of $\Delta^{++}$production, $\rho^{0}$ production, and background to the reaction

$$
\gamma p \rightarrow p \pi^{+} \pi^{-}
$$

were obtained by fitting a combination of phase-space and Breit-Wigner distributions to the data. The density distribution of events in the Dalitz plot $M^{2}\left(p \pi^{+}\right)$versus $M^{2}\left(\pi^{+} \pi^{-}\right)$was described by the expression

$$
\begin{aligned}
& d N\left(M^{2}\left(p \pi^{+}\right), M^{2}\left(\pi^{+} \pi^{-}\right), E_{\gamma}\right) \\
& =\left(a_{\Delta} \frac{B_{\Delta}\left(M\left(p \pi^{+}\right)\right)}{N_{\Delta}}+a_{\rho} \frac{B_{\rho}\left(M\left(\pi^{+} \pi^{-}\right)\right) W\left(\cos \theta_{H}\right) H_{\rho}\left(E_{\gamma}\right)}{N_{\rho}}\right. \\
& \left.+a_{\mathrm{PS}} \frac{B_{\mathrm{PS}}}{N_{\mathrm{PS}}}\right) \frac{S\left(E_{\gamma}\right)}{E_{\gamma} E_{\mathrm{c} . \mathrm{m} \cdot}{ }^{2}} d M^{2}\left(p \pi^{+}\right) \\
& \times d M^{2}\left(\pi^{+} \pi^{-}\right) d E_{\gamma \cdot} \quad \text { (A1) }
\end{aligned}
$$

Here, $a_{\Delta}, a_{\rho}$, and $a_{\mathrm{PS}}$ are the numbers of $\Delta^{++}, \rho$, and background events, respectively $\left(a_{\Delta}+a_{\rho}+a_{\mathrm{PS}}\right.$ is the total number of events), $M\left(p \pi^{+}\right)$is the effective mass of $p \pi^{+}, M\left(\pi^{+} \pi^{-}\right)$is the effective mass of $\pi^{+} \pi^{-}, E_{\mathrm{c} . \mathrm{m}}$. is the total c.m. energy, and $B_{\Delta}$ and $B_{\rho}$ are relativistic Breit-Wigner distributions with energy-dependent 
width as suggested by Jackson, ${ }^{37}$

$$
\begin{aligned}
& B(M)=\frac{M}{q(M)} \frac{\Gamma(M)}{\left(M^{2}-M_{0}^{2}\right)^{2}+M_{0}^{2} \Gamma^{2}(M)}, \\
& \Gamma(M)=\Gamma_{0}\left(\frac{q(M)}{q\left(M_{0}\right)}\right)^{3} \frac{\rho(M)}{\rho\left(M_{0}\right)} .
\end{aligned}
$$

$\Delta^{++}$production:

$$
\begin{aligned}
M & =M\left(p \pi^{+}\right), \\
B(M) & =B_{\Delta}\left(M\left(p \pi^{+}\right)\right), \\
M_{0} & =1236 \mathrm{MeV}, \\
\Gamma_{0} & =120 \mathrm{MeV}, \\
q(M) & =\text { three-momentum of the proton in the } p \pi^{+} \\
& \text {rest system, } \\
\rho(M)= & {\left[2.2 M_{\pi}^{2}+q^{2}(M)\right]^{-1}, } \\
M_{\pi} & =\text { pion mass. }
\end{aligned}
$$

$\rho$ production:

$$
\begin{aligned}
M & =M\left(\pi^{+} \pi^{-}\right), \\
B(M) & =B_{\rho}\left(M\left(\pi^{+} \pi^{-}\right)\right), \\
M_{0}= & M_{\rho} \text { (resonance mass), } \\
\Gamma_{0}= & 143 \mathrm{MeV}, \\
q(M)= & \text { three-momentum of the } \pi^{+} \text {in the } \pi \pi \text { rest } \\
& \text { system, } \\
\rho(M)= & {\left[q^{2}(M)+q^{2}\left(M_{0}\right)\right]^{-1} . }
\end{aligned}
$$

$B_{\mathrm{PS}}=$ const is the Lorentz-invariant phase-space distribution. $W\left(\cos \theta_{H}\right)=\frac{3}{4}\left[1-\rho_{00}+\left(3 \rho_{00}-1\right) \cos ^{2} \theta_{H}\right]$ describes the $\rho$ decay distribution in the helicity system. In this system the cosine of the decay angle, $\cos \theta_{H}$, can be expressed by $M^{2}\left(p \pi^{+}\right), M^{2}\left(\pi^{+} \pi^{-}\right)$, and $E_{\gamma}$. The parameter $\rho_{00}$ was determined from experiment. By introducing the factor $W\left(\cos \theta_{H}\right)$ the fits in the $p \pi^{+}$ mass distribution were improved considerably.

The density distribution was averaged over finite intervals of the photon energy. Appropriate weighting factors depending on the photon energy have been introduced:

$$
S\left(E_{\gamma}\right)=\text { photon spectrum. }
$$

The over-all factor $1 / E_{\gamma}$ is due to the flux factor appearing in the definition of a cross section. $H_{\rho}\left(E_{\gamma}\right)$ describes the photon-energy dependence of $B_{\rho}(=$ square of the matrix element for $\rho$ production). We used $H_{\rho}=E_{\gamma}{ }^{0.74}$, as determined by preliminary fits. The analogous factors for $\Delta^{++}$and phase space were assumed to be constant. Slight modifications of the photonenergy-dependent factors did not change the results outside errors.

The three contributions to the density distribution were normalized to unity. The normalization factors $N_{\Delta}, N_{\rho}$, and $N_{\mathrm{Ps}}$ were obtained by integration over the whole Dalitz plot and over the photon energy

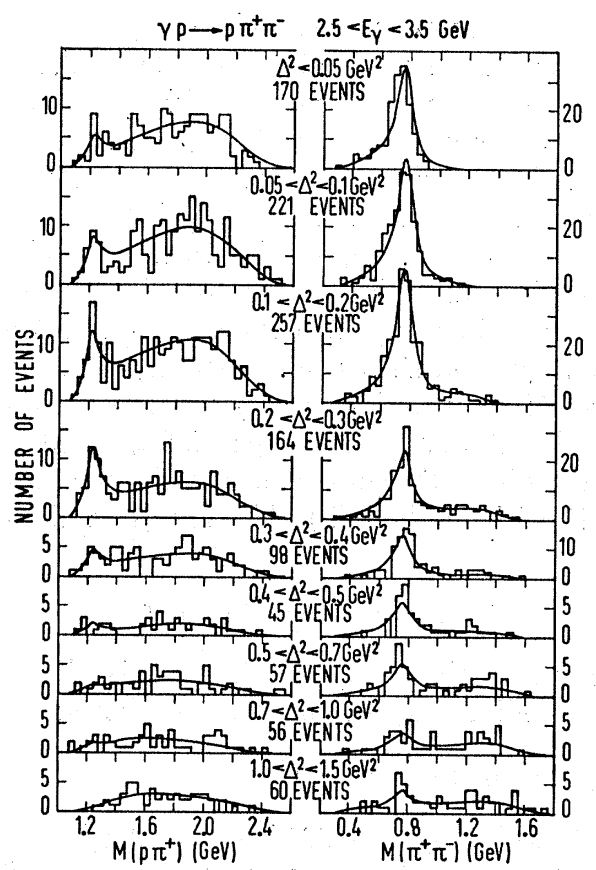

FIG. 40. Reaction $\gamma p \rightarrow p \pi^{+} \pi^{-}$for photon energies $2.5<E_{\gamma}$ $<3.5 \mathrm{GeV}$. Effective-mass distributions $M\left(p \pi^{+}\right)$and $M\left(\pi^{+} \pi^{-}\right)$ for various intervals of $\Delta^{2}$, the square of the momentum transfer between incoming and outgoing proton. The curves are explained in the Appendix.

interval considered, e.g.,

$$
\begin{aligned}
N_{\rho}=\int B_{\rho}\left(M\left(\pi^{+} \pi^{-}\right)\right) W\left(\cos \theta_{H}\right) H_{\rho}\left(E_{\gamma}\right) \\
\quad \times \frac{S\left(E_{\gamma}\right)}{E_{\gamma} E_{\mathrm{c} . \mathrm{m} .}{ }^{2}} d M^{2}\left(p \pi^{+}\right) d M^{2}\left(\pi^{+} \pi^{-}\right) d E_{\gamma} .
\end{aligned}
$$

Three different forms of the $\rho$ resonance, distribution were used: method (i) as described above, method (ii), same as above but using in (A1) and (A4)

$$
\left[M_{\rho} / M\left(\pi^{+} \pi^{-}\right)\right]^{4} B_{\rho}\left(M\left(\pi^{+} \pi^{-}\right)\right)
$$

instead of $B_{\rho}\left(M\left(\pi^{+} \pi^{-}\right)\right)$, and method (iii), same as above but replacing in (A1) and (A4) $B_{\rho}\left(M\left(\pi^{+} \pi^{-}\right)\right) W\left(\cos \theta_{H}\right)$ by

$$
\begin{aligned}
B_{\rho}\left(M\left(\pi^{+} \pi^{-}\right)\right) W\left(\cos \theta_{H}\right)+ & 2\left(\sqrt{ } a_{D}\right) D_{1}\left(M\left(\pi^{+} \pi^{-}\right), E_{\gamma}\right) \\
& +a_{D} D_{2}\left(M\left(\pi^{+} \pi^{-}\right), E_{\gamma}\right) .
\end{aligned}
$$

Here, $D_{2}$ is the contribution from a Drell-type background according to Ref. 39, $a_{D}$ is the relative strength of the Drell-type background, and $D_{1}$ is the interference term describing the interference between a diffractive $\rho$ amplitude and the Drell-type amplitudes. To calculate the Drell-type amplitudes experimental $\pi p$ phase shifts were used.

$D_{1}$ and $D_{2}$ are parametrizations of the interference term and the Drell background obtained after integration over all variables but $M\left(\pi^{+} \pi^{-}\right)$and $E_{\gamma}$. Method (iii), therefore, was used for determination of total cross sections only. 
The parameters $a_{\Delta}, a_{\rho}, a_{D}$, and $a_{\mathrm{PS}}$ were determined by fitting the density distribution (A1) to the experimental data. Both maximum-likelihood and leastsquares methods were used. ${ }^{35}$ To determine differential cross sections the data were subdivided into several intervals of the production angle and of $\Delta^{2}$, and the fitting procedure was applied for each interval separately. The kinematical limits of the density distribution, which are introduced by the cuts in $\Delta^{2}$, were taken into account. Figure 40 shows, for example, the mass distributions $M\left(p \pi^{+}\right)$and $M\left(\pi^{+} \pi^{-}\right)$for several intervals of $\Delta^{2}$ in the photon energy range $2.5<E_{\gamma}<3.5 \mathrm{GeV}$. The full curves are the sum of all contributions obtained from (A1) with method (ii). The fits are satisfactory.

\section{Decay Matrix Elements}

To determine the decay density matrix elements of the $\rho^{0}$ we modified expression (A1) as follows:

$$
\begin{aligned}
& d N\left(M^{2}\left(p \pi^{+}\right), M^{2}\left(\pi^{+} \pi^{-}\right), E_{\gamma}\right) \\
& =\left[a_{\Delta} \frac{B_{\Delta}\left(M\left(p \pi^{+}\right)\right)}{N_{\Delta}}+a_{\rho} \frac{B_{\rho}\left(M\left(\pi^{+} \pi^{-}\right)\right) H_{\rho}\left(E_{\gamma}\right)}{N_{\rho}} W_{\rho}(\theta, \phi)\right. \\
& \left.\quad+a_{\mathrm{PS}} \frac{B_{\mathrm{PS}}}{N_{\mathrm{PS}}} W_{\mathrm{PS}}(\theta, \phi)\right] \frac{S\left(E_{\gamma}\right)}{E_{\gamma} E_{\mathrm{c} . \mathrm{m} .}{ }^{2}} d M^{2}\left(p \pi^{+}\right) \\
& \quad \times d M^{2}\left(\pi^{+} \pi^{-}\right) d E_{\gamma} .
\end{aligned}
$$

$\theta$ and $\phi$ are the decay angles defined in Sec. III C. $W_{\rho}(\theta, \phi)$ is the $\rho$ decay distribution. $W_{\rho}$ is given by Eq. (9). $W_{\text {Ps }}$ gives a phenomenological description of the background angular distribution allowing an asymmetric term in $\cos \theta$ :

$$
\begin{array}{r}
W_{\mathrm{PS}}(\theta, \phi)=3 / 4 \pi\left[\frac{1}{2}\left(1-\rho_{00}{ }^{\prime}\right)+\frac{1}{2}\left(3 \rho_{00}{ }^{\prime}-1\right) \cos ^{2} \theta\right. \\
\left.-\rho_{1-1}{ }^{\prime} \sin ^{2} \theta \cos 2 \phi-\sqrt{2} \operatorname{Re} \rho_{10}{ }^{\prime} \sin 2 \theta \cos \phi\right] \\
+(\sqrt{3} / 4 \pi) 2 \rho^{\prime} \cos \theta .
\end{array}
$$

$W_{p}$ and $W_{\mathrm{PS}}$ are normalized to unity.
We determine the parameters $\rho_{00}, \rho_{1-1}, \operatorname{Re} \rho_{10}, \rho_{00}$, $\rho_{1-1}{ }^{\prime}, \operatorname{Re} \rho_{10}{ }^{\prime}$, and $\rho^{\prime}$ by a maximum-likelihood fit of Eq. (A6) to the data. The numbers of events $a_{\Delta}, a_{\rho}$, $a_{\mathrm{PS}}$, which were obtained by separate fits, were inserted as fixed parameters. $B_{\Delta}$ is not multiplied by a term depending on the decay angles $\theta$ and $\phi$. Since the expression $a_{\Delta} B_{\Delta} / N_{\Delta}$ contains no adjustable parameter, its contribution to the likelihood function is approximately constant and does not influence severely the determination of $\rho_{00}, \rho_{1-1}$, and $\operatorname{Re} \rho_{10}$.

\section{B. Fit of the Density Matrix for Reaction $\gamma p \rightarrow p \omega$}

The $\omega$ density matrix elements $\rho_{00}, \rho_{1-1}$, and $\operatorname{Re} \rho_{10}$ were determined by a fit to the experimental decay distribution. The decay distribution of events from reaction $\gamma p \rightarrow p \pi^{+} \pi^{-} \pi^{0}$ in a mass region of about 150 $\mathrm{MeV}$ width centered around the $\omega$ was described by the expression

$$
\begin{aligned}
d N\left(\cos \theta, \phi, M\left(\pi^{+} \pi^{-} \pi^{0}\right)\right)=n\left[a G_{\omega}\left(M\left(\pi^{+} \pi^{-} \pi^{0}\right)\right)\right. \\
\times W_{\omega}(\cos \theta, \phi)+(1-a) F_{\mathrm{PS}}\left(M\left(\pi^{+} \pi^{-} \pi^{0}\right)\right) \\
\left.\quad \times W_{\mathrm{PS}}(\cos \theta, \phi)\right] d \cos \theta d \phi d M\left(\pi^{+} \pi^{-} \pi^{0}\right) .
\end{aligned}
$$

Here, $n$ is the total number of events in the mass region used, $\theta$ and $\phi$ are the decay angles defined in Sec. IV, and $W_{\omega}(\cos \theta, \phi)$ is the $\omega$ decay distribution, given by Eq. (9). Since the decay distributions were found to be approximately symmetric in the mass regions near the $\omega$, we used Eq. (9) with different parameters $\rho_{00}{ }^{\prime}$, $\rho_{1-1}{ }^{\prime}$, and $\operatorname{Re} \rho_{10}{ }^{\prime}$ to describe the background decay distribution $W_{\mathrm{PS}}(\cos \theta, \phi) ; a$ is the fraction of $\omega$ events in the fit region; $a$ was inserted as a fixed parameter, $G_{\omega}$ is a normal distribution with appropriate width to describe the $\omega$ mass peak, and $F_{P S}$ is the Lorentz-invariant phase-space distribution. $G_{\omega}$ and $F_{\mathrm{PS}}$ are normalized to unity.

The parameters $\rho_{00}, \rho_{1-1}, \operatorname{Re} \rho_{10}, \rho_{00}{ }^{\prime}, \rho_{1-1}{ }^{\prime}$, and $\operatorname{Re} \rho_{10}{ }^{\prime}$ were determined by maximum-likelihood fits of (A8) to the data. 